\title{
Supporting Information: \\ Catalytic Arylboration of Spirocyclic Cyclobutenes: Rapid Access to Highly Substituted Spiro[3.n]alkanes
}

\author{
Amit Kumar Simlandy, Maoyun Lyu and M. Kevin Brown* \\ Indiana University, 800 E. Kirkwood Ave., Bloomington, IN 47401 \\ Email: brownmkb@indiana.edu
}

$\begin{array}{ll}\text { 1. General Information } & \text { S2 }\end{array}$

2. Reagents and Catalysts $\quad$ S3

3. Substrate and $\mathrm{Cu}-\mathrm{NHC}$ Synthesis $\quad$ S4

4. General Procedure $\quad$ S8

$\begin{array}{ll}\text { 5. Optimization } & \mathrm{S} 10\end{array}$

6. Characterization Data $\quad \mathrm{S} 12$

$\begin{array}{lll}\text { 7. Further Transformations } & \text { S27 }\end{array}$

8. Single Crystal X-ray Diffraction Analysis of $\mathbf{2 6}$ and $\mathbf{4 1} \quad$ S34

$\begin{array}{ll}\text { 9. Spectra } & \text { S36 }\end{array}$

10. References $\quad$ S86 


\section{General Information:}

Infrared (IR) spectra were recorded on a Bruker Tensor II FT-IR Spectrometer, $v_{\max }$ in $\mathrm{cm}^{-1}$. Bands are characterized as broad (br), strong (s), medium (m), and weak (w). ${ }^{1} \mathrm{H}$ NMR spectra were recorded at room temperature unless otherwise noted on a Varian I400 (400 MHz), Varian VXR400 (400 MHz), Varian I500 (500 MHz), or a Varian I600 (600 MHz) spectrometer. Chemical shifts are reported in ppm from tetramethylsilane with the residual solvent resonance as the internal standard $\left(\mathrm{CHCl}_{3}: \delta 7.26 \mathrm{ppm}\right)$. Data are reported as follows: chemical shift, multiplicity ( $\mathrm{s}=$ singlet, $\mathrm{d}=$ doublet, $\mathrm{t}=$ triplet, $\mathrm{q}=$ quartet, $\mathrm{br}=$ broad, $\mathrm{m}=$ multiplet $)$, coupling constants $(\mathrm{Hz})$, and integration. ${ }^{13} \mathrm{C}$ NMR spectra were recorded on a Varian $\mathrm{I} 400(100 \mathrm{MHz})$, Varian VXR400 (100 MHz), Varian I500 (125 MHz), or a Bruker B500 (125 MHz) spectrometer with complete proton decoupling. Chemical shifts are reported in ppm from tetramethylsilane with the solvent resonance as the internal standard $\left(\mathrm{CDCl}_{3}: \delta 77.16 \mathrm{ppm}\right) .{ }^{19} \mathrm{~F}$ NMR spectra were recorded on Varian VXR400 (375 MHz) spectrometer. High-resolution mass spectrometry (HRMS) was performed on either a Waters/Micromass LCT Classic (ESI-TOF) or a Thermo Electron Corporation MAT 95XP-Trap (GC/MS). The diastereomeric ratios were determined using NMR analysis of unpurified reaction mixtures. GC analyses were performed by means of Agilent 6850 Gas Chromatograph equipped with Agilent 19091Z-413E, 30 m x $320 \mu \mathrm{m}$ x 0.25 $\mu \mathrm{m}$ column. Helium was used as the GC carrier gas and maintained at a constant flow rate of 25.0 $\mathrm{mL} / \mathrm{min}$. The capillary column was held for 1.0 minutes at the initial temperature $\left(60{ }^{\circ} \mathrm{C}\right)$ and subsequently ramped at a rate of $25^{\circ} \mathrm{C} / \mathrm{min}$ to a final temperature of $300{ }^{\circ} \mathrm{C}$. Total run time was $9.60 \mathrm{~min}$.

Unless otherwise noted, all reactions have been carried out with distilled and degassed solvents under an atmosphere of dry $\mathrm{N}_{2}$ in oven- $\left(135^{\circ} \mathrm{C}\right)$ and flame-dried glassware with standard vacuumline techniques. Toluene was purified under a positive pressure of dry argon by passage through columns of activated alumina and Q5 (Grubbs apparatus). All work-up and purification procedures were carried out with reagent grade solvents (purchased from Sigma-Aldrich) in air. Standard column chromatography techniques using ZEOprep 60/40-63 $\mu \mathrm{m}$ silica gel was used for purification. 


\section{Reagents and Catalysts:}

tert-butyl 2-oxo-7-azaspiro[3.5]nonane-7-carboxylate was purchased from Ambeed and used as received.

tert-Butyl 7-azaspiro[3.5]non-1-ene-7-carboxylate was synthesized in accordance with literature procedure. ${ }^{1}$

tert-Butyl 2-azaspiro[3.3]hept-5-ene-2-carboxylate was synthesized in accordance with literature procedure. ${ }^{1}$

2-Benzyl-2-azaspiro[3.3]hept-5-ene was synthesized in accordance with literature procedure. ${ }^{1}$ tert-Butyl 6-azaspiro[3.4]oct-1-ene-6-carboxylate was synthesized in accordance with literature procedure. $^{1}$

Bromobenzene was purchased from Sigma-Aldrich and purified via neat filtration through a 2$\mathrm{cm}$ pad of dry silica in a 5.75-inch pipet prior to use.

Ethyl 4-bromobenzoate was purchased from Sigma-Aldrich and purified via neat filtration through a 2- cm pad of dry silica in a 5.75-inch pipet prior to use.

1-Bromo-2-methylbenzene was purchased from Oakwood and purified via neat filtration through a 2- cm pad of dry silica in a 5.75-inch pipet prior to use.

5-Bromobenzo[d][1,3]dioxole was purchased from Ambeed and purified via neat filtration through a $2-\mathrm{cm}$ pad of dry silica in a 5.75-inch pipet prior to use.

5-Bromo-2-methoxypyridine was purchased from Combi-Blocks and used as received.

2-Bromo-6-methoxypyridine was purchased from Combi-Blocks and used as received.

3-Bromo-6-methoxy-2-methylpyridine was purchased from Ambeed and used as received.

5-Bromo-2-(piperidin-1-yl)pyridine was purchased from AOB Chem and used as received.

4-(5-Bromopyrimidin-2-yl)morpholine was purchased from Combi-Blocks and used as received.

4-Bromo-2-methoxypyridine was purchased from Ambeed and used as received.

5-Bromobenzofuran was purchased from Combi-Blocks and used as received.

5-Bromo-1-methyl-1H-pyrrolo[2,3-b]pyridine was synthesized in accordance with literature procedure. $^{2}$

1-Bromo-4-chlorobenzene was purchased from Sigma-Aldrich and used as received. 
Bis(pinacolato)diboron was purchased from Oakwood and purified via recrystallization from pentane prior to use.

Ethyl magnesium bromide (3.0 $\mathrm{M}$ in diethyl ether) was purchased from Sigma-Aldrich and used as received.

Tris(acetylacetonato)iron(III) was purchased from Sigma-Aldrich and used as received.

Potassium bis(trimethylsilyl)amide (1.0 M in THF) was purchased from Sigma-Aldrich and used as received.

Phenyllithium solution (1.9 $\mathrm{M}$ in $\mathrm{Di}^{n}$ butylether) was purchased from Sigma-Aldrich and used as received.

Diethyl chlorophosphate was purchased from Oakwood and used as received.

1,3,5-Trimethylbenzene was purchased from Sigma-Aldrich and purified via column chromatography (100\% pentane) and stored over activated $4 \AA$ molecular sieves.

$\boldsymbol{N}, \boldsymbol{N}$-Dimethylacetamide (DMA) was purchased from Sigma-Aldrich in a Sure-Seal ${ }^{\mathrm{TM}}$ bottle and used as received.

Nickel(II) chloride ethylene glycol dimethyl ether complex $\left(\mathrm{Ni}(\mathrm{DME}) \mathrm{Cl}_{2}\right)$ was purchased from Strem and used as received.

SPhosPdG3 was synthesized in accordance with literature procedure. ${ }^{3}$

Potassium tert-butoxide was purchased from Oakwood and used as received.

Sodium tert-butoxide was purchased from Strem and used as received.

\section{General Procedure for Substrate and Cu-NHC Synthesis:}

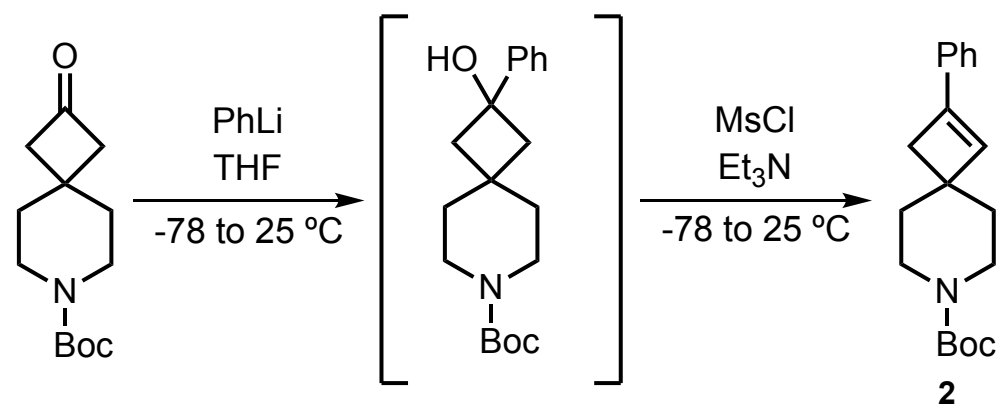

In a flame-dried round bottom flask, tert-butyl 2-oxo-7-azaspiro[3.5]nonane-7-carboxylate (1.0 g, $4.18 \mathrm{mmol}, 1.0$ equiv.) was taken in $20 \mathrm{~mL}$ THF under positive pressure of nitrogen. $\mathrm{PhLi}$ (1.9 $\mathrm{mL}, 4.18 \mathrm{mmol}, 1.0$ equiv.) was added dropwise to the flask at $-78^{\circ} \mathrm{C}$ and stirred for $1 \mathrm{~h}$ at $-78^{\circ} \mathrm{C}$ 
then at $25^{\circ} \mathrm{C}$ for another $1 \mathrm{~h}$. The flask was again cooled to $-78^{\circ} \mathrm{C}$, methane sulfonyl chloride $(1.3$ $\mathrm{mL}, 16.7 \mathrm{mmol}, 4.0$ equiv.) and triethylamine (4.6 mL, $33.4 \mathrm{mmol}, 8.0$ equiv.) were added and allowed to warm to room temperature over $3 \mathrm{~h}$. The reaction mixture was quenched by the addition of $10 \mathrm{~mL}$ water, aqueous layer was extracted with EtOAc $(3 \times 20 \mathrm{~mL})$. Combined organic layer was dried over $\mathrm{Na}_{2} \mathrm{SO}_{4}$, concentrated and purified using silica-gel column chromatography to obtain the cyclobutene 2 as white solid (730 mg, 58\% yield).

IR (neat): 1691 (s), 1172 (w), 752 (w) cm ${ }^{-1} ;{ }^{1} \mathbf{H}$ NMR (500 MHz, CDCl $): \delta 7.37-7.31$ (m, 4H), $7.27-7.24(\mathrm{~m}, 1 \mathrm{H}), 6.51(\mathrm{~s}, 1 \mathrm{H}), 3.62-3.57(\mathrm{~m}, 2 \mathrm{H}), 3.34-3.29(\mathrm{~m}, 2 \mathrm{H}), 2.53(\mathrm{~s}, 2 \mathrm{H}), 1.69-$ 1.59 (m, 4H), 1.48 (s, 9H); ${ }^{13} \mathbf{C}$ NMR (126 MHz, $\left.\mathbf{C D C l}_{3}\right): \delta 155.2,144.0,134.9,133.4,128.5$, 128.0, 124.6, 79.4, 42.6, 39.5, 35.6, 28.6; HRMS (ESI+): Calculated for $\mathrm{C}_{19} \mathrm{H}_{25} \mathrm{O}_{2} \mathrm{NNa}[\mathrm{M}+\mathrm{Na}]+$ : 322.1778, Found: 322.1777.

tert-Butyl 2-(benzo[d][1,3]dioxol-5-yl)-7-azaspiro[3.5]non-1-ene-7-carboxylate was synthesized following the above procedure. IR (neat): $1687(\mathrm{~s}), 1243(\mathrm{~s}), 1038(\mathrm{w})$
$\mathrm{cm}^{-1},{ }^{1} \mathbf{H} \mathbf{N M R}\left(\mathbf{5 0 0} \mathbf{M H z}, \mathbf{C D C l}_{3}\right): \delta 6.86(\mathrm{~d}, J=1.6 \mathrm{~Hz}, 1 \mathrm{H}), 6.81(\mathrm{~d}, J=8.0 \mathrm{~Hz}$,
$1 \mathrm{H}), 6.76(\mathrm{~d}, J=7.9 \mathrm{~Hz}, 1 \mathrm{H}), 6.35(\mathrm{~s}, 1 \mathrm{H}), 5.95(\mathrm{~s}, 2 \mathrm{H}), 3.60-3.55(\mathrm{~m}, 2 \mathrm{H}), 3.33-$
$3.28(\mathrm{~m}, 2 \mathrm{H}), 2.47(\mathrm{~s}, 2 \mathrm{H}), 1.66-1.58(\mathrm{~m}, 4 \mathrm{H}), 1.47(\mathrm{~s}, 9 \mathrm{H}) ;{ }^{13} \mathbf{C} \mathbf{N M R}(\mathbf{1 2 6} \mathbf{M H z}$,
$\left.\mathbf{C D C l}_{3}\right): \delta 155.2,147.9,147.5,143.5,131.4,129.6,118.6,108.3,104.9,101.2,79.4$,
$42.3,39.6,35.6,28.6 ; \mathbf{H R M S}(\mathbf{E S I}+):$ Calculated for $\mathrm{C}_{20} \mathrm{H}_{25} \mathrm{O}_{4} \mathrm{NNa}[\mathrm{M}+\mathrm{Na}]+$ :
366.1676, Found: 366.1677.

tert-Butyl 2-(4-(trifluoromethyl)phenyl)-7-azaspiro[3.5]non-1-ene-7-carboxylate was

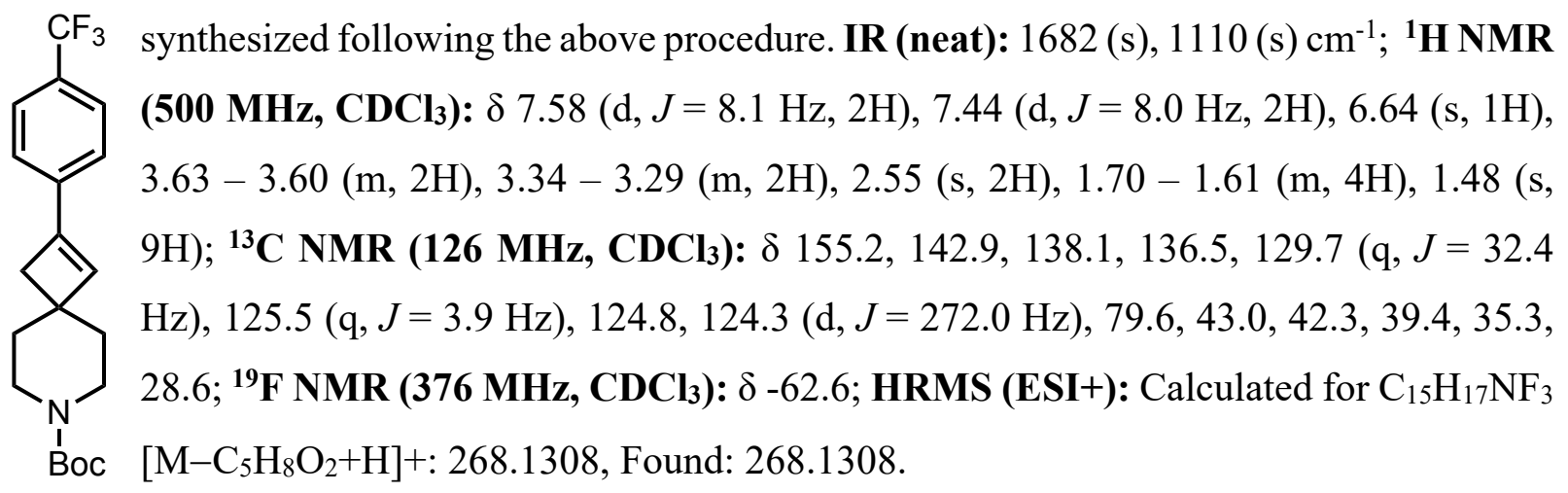




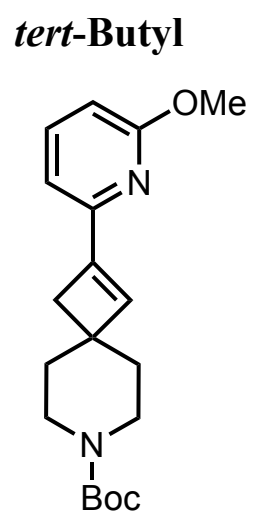
synthesized following the above procedure. ${ }^{1} \mathrm{H}$ NMR (500 $\left.\mathbf{~ M H z}, \mathrm{CDCl}_{3}\right)$ : IR (neat): $1692(\mathrm{~s}), 1244(\mathrm{~s}) \mathrm{cm}^{-1} ; \delta 7.52(\mathrm{t}, J=7.7 \mathrm{~Hz}, 1 \mathrm{H}), 6.83(\mathrm{~d}, J=7.3 \mathrm{~Hz}$, 1H), $6.77(\mathrm{~s}, 1 \mathrm{H}), 6.62(\mathrm{~d}, J=8.3 \mathrm{~Hz}, 1 \mathrm{H}), 3.94(\mathrm{~s}, 3 \mathrm{H}), 3.62-3.57$ (m, 2H), 3.35 - $3.30(\mathrm{~m}, 2 \mathrm{H}), 2.56(\mathrm{~s}, 2 \mathrm{H}), 1.71-1.61$ (m, 4H), 1.47 (s, 9H); ${ }^{13} \mathrm{C}$ NMR (126 MHz, $\left.\mathbf{C D C l}_{3}\right): \delta 163.9,155.2,150.6,144.1,138.8,138.0,112.6,110.0,79.5$, 53.2, 48.4, 43.0, 39.4, 35.3, 28.6; HRMS (ESI+): Calculated for $\mathrm{C}_{19} \mathrm{H}_{27} \mathrm{O}_{3} \mathrm{~N}_{2}$ $[\mathrm{M}+\mathrm{H}]+:$ 331.2016, Found: 331.2018.

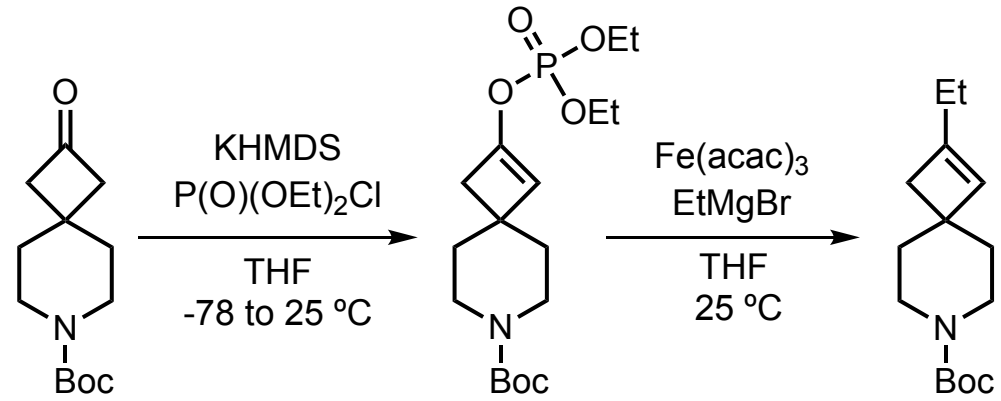

tert-butyl 2-((diethoxyphosphoryl)oxy)-7-azaspiro[3.5]non-1-ene-7-carboxylate was prepared according to modified literature procedure. ${ }^{4}$

IR (neat): 1693 (s), 1275 (w), 1049 (s) cm ${ }^{-1}$; ${ }^{1} \mathbf{H}$ NMR (400 MHz, CDCl $\mathbf{3}$ ): $\delta 5.27$ (d, $J=0.9 \mathrm{~Hz}$, $1 \mathrm{H}), 4.22-4.14(\mathrm{~m}, 4 \mathrm{H}), 3.56-3.50(\mathrm{~m}, 2 \mathrm{H}), 3.28-3.21(\mathrm{~m}, 2 \mathrm{H}), 2.48(\mathrm{~d}, J=1.4 \mathrm{~Hz}, 2 \mathrm{H}), 1.63$ - 1.52 (m, 4H), 1.45 (s, 9H), $1.36(\mathrm{td}, J=7.1,1.1 \mathrm{~Hz}, 6 \mathrm{H}) ;{ }^{13} \mathbf{C}$ NMR (126 MHz, CDCl 3$): ~ \delta ~ 155.1$, $143.2(\mathrm{~d}, J=9.0 \mathrm{~Hz}), 115.6(\mathrm{~d}, J=7.3 \mathrm{~Hz}), 79.5,64.7(\mathrm{~d}, J=6.0 \mathrm{~Hz}), 43.8$ (d, $J=5.6 \mathrm{~Hz}), 42.8$, $38.5,35.4,28.6,16.2(\mathrm{~d}, J=6.5 \mathrm{~Hz})$.

tert-butyl 2-ethyl-7-azaspiro[3.5]non-1-ene-7-carboxylate was prepared according to modified literature procedure. ${ }^{5}$

IR (neat): 1697 (s), 1174 (w) cm ${ }^{-1} ;{ }^{1} \mathbf{H}$ NMR (400 MHz, CDCl $): \delta 5.89$ (t, $\left.J=1.7 \mathrm{~Hz}, 1 \mathrm{H}\right), 3.53$ $-3.49(\mathrm{~m}, 2 \mathrm{H}), 3.27-3.21(\mathrm{~m}, 2 \mathrm{H}), 2.11(\mathrm{~s}, 2 \mathrm{H}), 2.04-1.98(\mathrm{~m}, 2 \mathrm{H}), 1.56-1.50(\mathrm{~m}, 4 \mathrm{H}), 1.46$ (s, 9H), 1.00 (t, $J=7.4 \mathrm{~Hz}, 3 \mathrm{H}) ;{ }^{13} \mathbf{C} \mathbf{N M R}\left(126 \mathbf{~ M H z}, \mathbf{C D C l}_{3}\right): \delta 155.2,149.9,132.6,79.3,42.5$, 41.7, 35.8, 28.6, 24.3, 11.1; HRMS (ESI+): Calculated for $\mathrm{C}_{15} \mathrm{H}_{25} \mathrm{O}_{2} \mathrm{NNa}[\mathrm{M}+\mathrm{Na}]+:$ : 274.1778, Found: 274.1778 . 


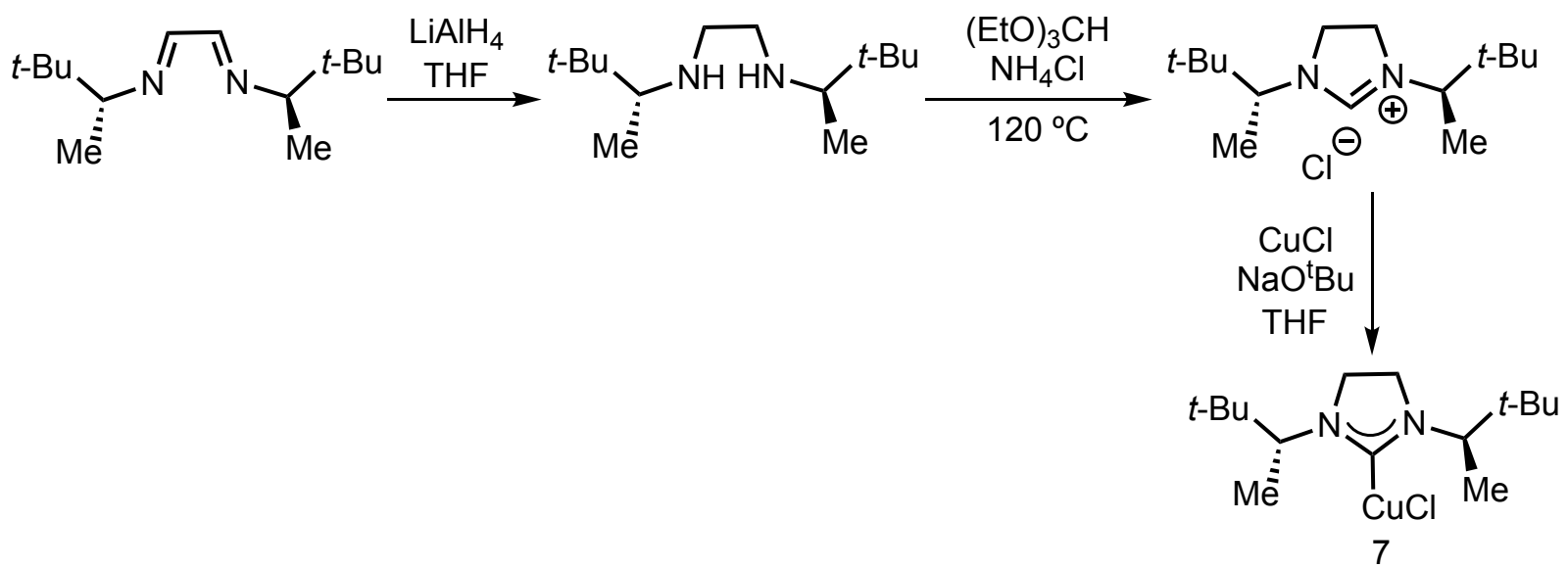

\section{1,3-Bis((R)-3,3-dimethylbutan-2-yl)-4,5-dihydro-1H-imidazol-3-ium chloride:}

$(1 E, 2 E)-N^{1}, N^{2}$-bis((R)-3,3-dimethylbutan-2-yl)ethane-1,2-diimine was prepared according to literature procedure. ${ }^{6}$

In an oven-dried round bottom flask, diimine (1.12 g, $5.0 \mathrm{mmol}, 1.0$ equiv.) was taken in $20 \mathrm{~mL}$ THF under $\mathrm{N}_{2}$ atmosphere. The solution was cooled down to $0^{\circ} \mathrm{C}$ (ice-bath) and lithium aluminum hydride (380 mg, $10.0 \mathrm{mmol}, 2.0$ equiv.) was added portion wise. After stirring at room temperature for $24 \mathrm{~h}$, the reaction mixture was quenched by the addition of water $(10 \mathrm{~mL})$ and 2 $\mathrm{M} \mathrm{KOH}$ solution $(20 \mathrm{~mL})$ at $0^{\circ} \mathrm{C}$ (ice-bath). The organic layer was separated, and the aqueous phase extracted with EtOAc $(3 \times 20 \mathrm{~mL})$. The combined organic layers were washed with water $(30 \mathrm{~mL})$, brine $(15 \mathrm{~mL})$, dried over $\mathrm{Na}_{2} \mathrm{SO}_{4}$, gravity filtered, and concentrated to obtain the diamine (1.0 g, 88\% yield). Crude diamine was carried forward to the next step without any further purification. ${ }^{1} \mathbf{H}$ NMR (400 MHz, $\left.\mathbf{C D C l}_{3}\right): \delta 2.94-2.89(\mathrm{~m}, 2 \mathrm{H}), 2.49-2.44(\mathrm{~m}, 2 \mathrm{H}), 2.21$ (q, $J$ $=6.4 \mathrm{~Hz}, 2 \mathrm{H}), 1.37(\mathrm{~s}, 2 \mathrm{H}), 0.98(\mathrm{~d}, J=6.4 \mathrm{~Hz}, 6 \mathrm{H}), 0.88(\mathrm{~s}, 18 \mathrm{H})$.

In an oven-dried round bottom flask, diamine ( $1.0 \mathrm{~g}, 4.38 \mathrm{mmol}, 1.0$ equiv.), $\mathrm{NH}_{4} \mathrm{Cl}(260 \mathrm{mg}, 4.80$ mmol, 1.1 equiv.) were taken. The vial was then inerted with three evacuation/backfill $\left(\mathrm{N}_{2}\right)$ cycles, sealed with a septum and triethyl orthoformate $(1.8 \mathrm{~mL}, 10.9 \mathrm{mmol}, 2.5$ equiv.) was added. The flask was then connected to a short distillation apparatus and heated at $120^{\circ} \mathrm{C}$ for $2 \mathrm{~h}$. The reaction mixture was cooled down to $70{ }^{\circ} \mathrm{C}$ and $5.0 \mathrm{~mL}$ EtOAc was added. Solvent was removed after cooling down to room temperature to obtain a solid. The solid was washed with $\mathrm{Et}_{2} \mathrm{O}(4 \times 3 \mathrm{~mL})$ and pentane $(4 \times 3 \mathrm{~mL})$ to obtain the salt as yellow solid (1.0 g, 83\% yield). IR (neat): 2958 (w), 1635 (s), 1133 (s) cm ${ }^{-1}$; ${ }^{1}$ H NMR (400 MHz, CDCl $): \delta 10.26$ (s, 1H), $4.01-3.92$ (m, 6H), 1.37 (d, $J=7.1 \mathrm{~Hz}, 6 \mathrm{H}), 1.01$ (s, 18H); ${ }^{13} \mathbf{C}$ NMR (101 MHz, CDCl 3$): \delta 159.7,62.8,48.0,35.4,26.9$, 14.0; HRMS (ESI+): Calculated for $\mathrm{C}_{15} \mathrm{H}_{31} \mathrm{~N}_{2}$ [M-Cl] $]^{+}: 239.2482$, Found: 239.2484. 
(1,3-bis((R)-3,3-dimethylbutan-2-yl)imidazolidin-2-yl)copper(I) chloride (7): In an $\mathrm{N}_{2}$-filled glovebox, a flame-dried $50 \mathrm{~mL}$ round bottom flask was charged with Chiral SI ${ }^{t} \mathrm{BuMe} . \mathrm{HCl}(1.00$ equiv), $\mathrm{CuCl}$ (1.00 equiv), and $\mathrm{NaO}^{t} \mathrm{Bu}$ (1.00 equiv). The flask was sealed with a septum and removed from the glovebox. Dry THF $(4.0 \mathrm{~mL})$ was added, and the solution was stirred at room temperature $\left(22^{\circ} \mathrm{C}\right)$ for $18 \mathrm{~h}$. At this time, the reaction was diluted with $\mathrm{CH}_{2} \mathrm{Cl}_{2}(\sim 20 \mathrm{~mL})$ and vacuum filtered through a $2 \mathrm{~cm}$ pad of celite. The filter cake was washed generously with $\mathrm{CH}_{2} \mathrm{Cl}_{2}$ $(\sim 50 \mathrm{~mL})$ and the filtrate concentrated to $\sim 5 \mathrm{~mL}$ total volume. The crude material was filtered through a silica plug $(1.75 \mathrm{~cm} \times 12 \mathrm{~cm})$ eluting with $\mathrm{CH}_{2} \mathrm{Cl}_{2}(100 \mathrm{~mL})$ into a $200 \mathrm{~mL}$ round bottom flask. The filtrate was concentrated to $\sim 2 \mathrm{~mL}$ and triturated with hexanes. The solvent was removed under vacuum to obtain 7 as a white solid that was quickly transferred to the glovebox for storage. IR (neat): 2955 (w), 1476 (s), 1448 (s), 1264 (s) cm ${ }^{-1} ;{ }^{1}$ H NMR (500 MHz, CDCl $) \delta$ $4.09(\mathrm{dd}, J=7.2,1.9 \mathrm{~Hz}, 2 \mathrm{H}), 3.56-3.51(\mathrm{~m}, 4 \mathrm{H}), 1.23(\mathrm{~d}, J=7.1 \mathrm{~Hz}, 6 \mathrm{H}), 0.99(\mathrm{~s}, 18 \mathrm{H}) ;{ }^{13} \mathrm{C}$ NMR (126 MHz, $\left.\mathbf{C D C l}_{3}\right): \delta$ 202.3, 64.7, 46.2, 35.5, 27.8, 13.9; HRMS (ESI+): Calculated for $\mathrm{C}_{30} \mathrm{H}_{60} \mathrm{~N}_{4} \mathrm{ClCu}_{2}$ [2M-Cl]+: 641.3061, Found: 641.3047.

\section{General Procedure for Arylboration}

\section{Procedure A:}

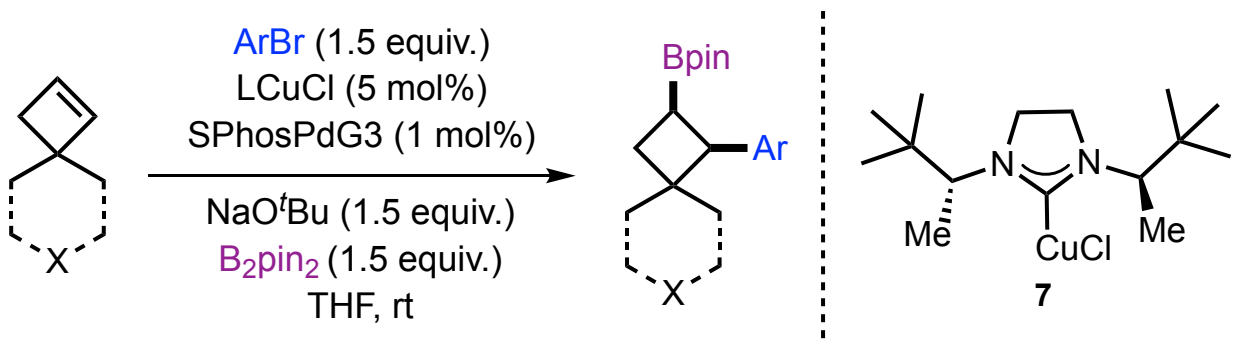

In an $\mathrm{N}_{2}$-filled glovebox, to an oven-dried 13 x $100 \mathrm{~mm}$ screw-capped vial was added bis(pinacolato)diboron (76.0 mg, $0.3 \mathrm{mmol}, 1.50$ equiv.), sodium tert-butoxide $(29.0 \mathrm{mg}, 0.3$ mmol, 1.5 equiv.), SPhosPdG3 (1.6 mg, $0.002 \mathrm{mmol}, 1.0 \mathrm{~mol} \%)$ and NHC-CuCl 7 (3.4 mg, 0.01 mmol, $5.0 \mathrm{~mol} \%$ ). Vial was sealed with septa and removed from the glovebox. A solution of alkene ( $0.2 \mathrm{mmol}, 1.0$ equiv.) and aryl bromide ( $0.3 \mathrm{mmol}, 1.5$ equiv. $)$ in $2.0 \mathrm{~mL}$ THF was added to the reaction vial. The septum was then quickly replaced by a Teflon- lined screw cap and the reaction was stirred at $25^{\circ} \mathrm{C}$ for $18 \mathrm{~h}$. The reaction was quenched upon the addition of brine $(5.0 \mathrm{~mL})$, and the mixture was extracted with EtOAc $(3 \times 5.0 \mathrm{~mL})$. The organic layer was washed with $1 \mathrm{M} \mathrm{KOH}$ 
( 2 x $5.0 \mathrm{~mL}$ ), dried over $\mathrm{Na}_{2} \mathrm{SO}_{4}$, gravity filtered, and concentrated. 1,3,5-Trimethylbenzene was added as an internal standard and a small aliquot was analyzed via ${ }^{1} \mathrm{H}-\mathrm{NMR}$. Purified by silica-gel column chromatography.

\section{Procedure B:}

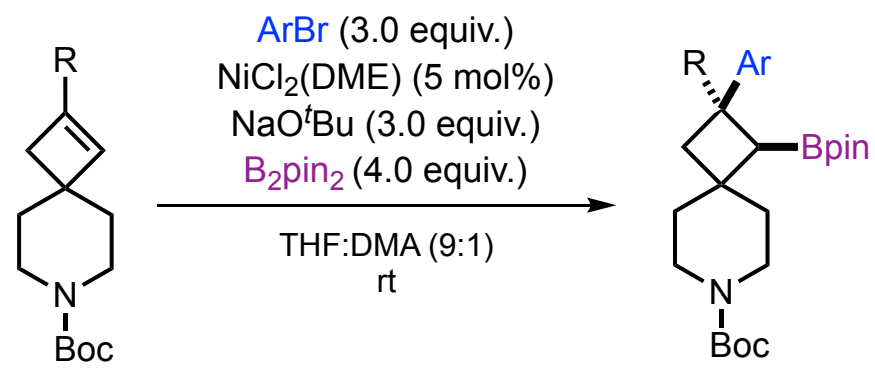

In an $\mathrm{N}_{2}$-filled glovebox, to an oven-dried $13 \times 100 \mathrm{~mm}$ screw-capped vial was added bis(pinacolato)diboron (203 $\mathrm{mg}, 0.8 \mathrm{mmol}, 4.00$ equiv.) and sodium tert-butoxide (58 $\mathrm{mg}, 0.6$ mmol, 3.0 equiv.). In a separate oven-dried 2-dram vial, $\mathrm{Ni}(\mathrm{DME}) \mathrm{Cl}_{2}(8.20 \mathrm{mg}, 0.0375 \mathrm{mmol})$ was added. Both vials were sealed with septa and removed from the glovebox. A solution of alkene ( $0.2 \mathrm{mmol}, 1.0$ equiv.) and aryl bromide ( $0.6 \mathrm{mmol}, 3.0$ equiv.) in $1.8 \mathrm{~mL}$ THF was added to the reaction vial. DMA $(0.75 \mathrm{~mL})$ was then added to the vial containing $\mathrm{Ni}(\mathrm{DME}) \mathrm{Cl}_{2}(0.05 \mathrm{M}$ in $\left.\mathrm{Ni}(\mathrm{DME}) \mathrm{Cl}_{2}\right)$ to prepare the catalyst solution. The catalyst solution $(0.2 \mathrm{~mL})$ was then added to the reaction vial ( $5 \mathrm{~mol} \%$ catalyst loading). The septum was then quickly replaced by a Teflonlined screw cap and the reaction was stirred at $25^{\circ} \mathrm{C}$ for $18 \mathrm{~h}$. The reaction was quenched upon the addition of brine $(5.0 \mathrm{~mL})$, and the mixture was extracted with EtOAc $(3 \times 5.0 \mathrm{~mL})$. The organic layer was washed with $1 \mathrm{M} \mathrm{KOH}(2 \times 5.0 \mathrm{~mL})$, dried over $\mathrm{Na}_{2} \mathrm{SO}_{4}$, gravity filtered, and concentrated. 1,3,5-Trimethylbenzene was added as an internal standard and a small aliquot was analyzed via ${ }^{1} \mathrm{H}-\mathrm{NMR}$. Purified by silica-gel column chromatography. 


\section{Optimization:}

\section{Optimization of $\mathrm{Cu}$-catalyzed arylboration:}

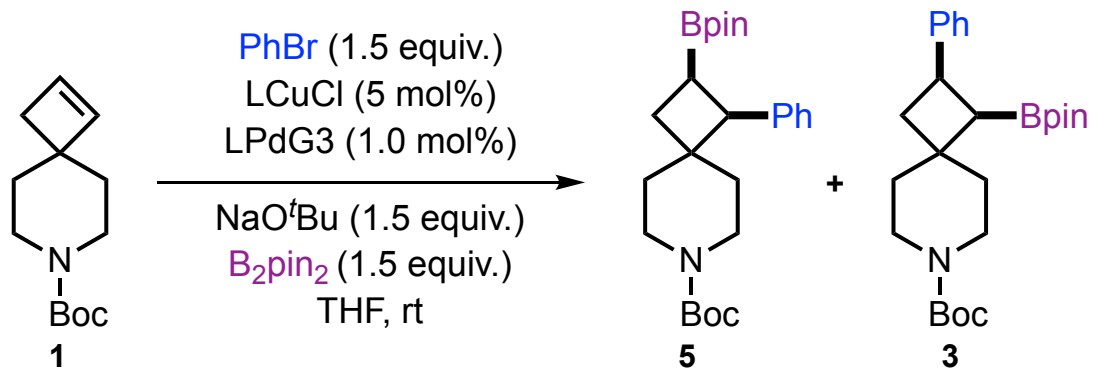

\begin{tabular}{clc}
\hline LCuCl & LPdG3 & yield \% (5:3) \\
\hline IMesCuCl & APhos & $86(5.6: 1)$ \\
IPrCuCl & APhos & $74(2.9: 1)$ \\
SIMesCuCl & APhos & $76(5.3: 1)$ \\
ItBuCuCl & APhos & $34(3.4: 1)$ \\
ICyCuCl & APhos & $69(8.9: 1)$ \\
\hline
\end{tabular}

\begin{tabular}{ccc}
\hline LCuCl & LPdG3 & yield \% (5:3) \\
\hline IMesCuCl & RuPhos & $65(4.4: 1)$ \\
\hline $\mathrm{MesCuCl}$ & $\mathrm{Cy}_{2}$ APhos & $84(11.5: 1)$ \\
\hline $\mathrm{MesCuCl}$ & SPhos & $88(12: 1)$ \\
\hline $\mathrm{MesCuCl}$ & $\mathrm{P}(\mathrm{tBu})_{3}$ Phos & $25(4.9: 1)$ \\
\hline $\mathrm{MesCuCl}$ & dppfPhos & $28(7: 1)$ \\
\hline
\end{tabular}

\section{Chiral Ligand Screening:}<smiles></smiles>

$56 \%(14: 1 \mathrm{rr}), 64: 36 \mathrm{er}$

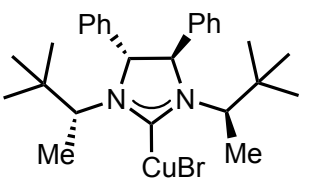

$67 \%(16: 1 \mathrm{rr}), 62: 38 \mathrm{er}$

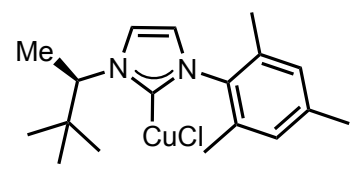

$57 \%(12: 1 \mathrm{rr}), 74: 26$ er<smiles></smiles><smiles></smiles>

$89 \%$ (27:1 rr), 60:40 er

$89 \%$ (27:1 rr), 53:47 er

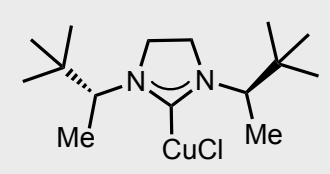

93\% (29:1 rr), 69:31 er $78 \%$ isolated, $>20: 1 \mathrm{rr}$<smiles></smiles>

$88 \%(17: 1 \mathrm{rr}), 50: 50 \mathrm{er}$<smiles>CN1C(=C(Cl)Cl)N2C(=N[C@@H](c3ccccc3)[C@H]2c2ccccc2)c2ccccc21</smiles>

$59 \%$ alkene remaining $3: 1 \mathrm{rr}$ 


\section{Optimization of Nickel catalyzed arylboration:}

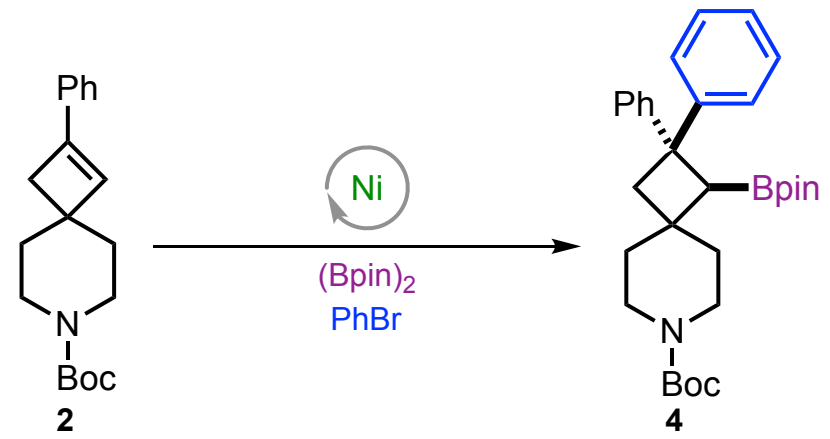

\begin{tabular}{|c|c|c|c|}
\hline $\begin{array}{c}\mathrm{PhBr}(1.5 \text { equiv. }) \\
\mathrm{NiCl}_{2} \text { (DME) }(15 \text { mol } \%) \\
\mathrm{KOMe}(2.0 \text { equiv. }) \\
\mathrm{B}_{2} \mathrm{pin}_{2}(2.0 \text { equiv. }) \\
\text { Toluene, } 60^{\circ} \mathrm{C} \\
<10 \% \text { pdt }\end{array}$ & $\begin{array}{c}\mathrm{PhBr}(1.5 \text { equiv. }) \\
\mathrm{NiCl}_{2}(\mathrm{DME})(5 \text { mol\%) } \\
\mathrm{LiO}^{t} \mathrm{Bu}(1.5 \text { equiv. }) \\
\mathrm{B}_{2} \mathrm{pin}_{2}(2.0 \text { equiv. }) \\
\mathrm{THF}, \mathrm{rt} \\
<10 \% \text { pdt }\end{array}$ & $\begin{array}{c}\mathrm{PhBr}(1.5 \text { equiv.) } \\
\mathrm{NiCl}_{2} \text { (DME) (5 mol\%) } \\
\mathrm{NaO}^{t} \mathrm{Bu}(1.5 \text { equiv.) } \\
\mathrm{B}_{2} \mathrm{pin}_{2}(2.0 \text { equiv.) } \\
\mathrm{THF}: \mathrm{DMA}(9: 1), \mathrm{rt} \\
\mathbf{4 4 \% ,} \mathbf{2 0 : 1} \boldsymbol{r r} \\
\mathbf{( 6 9 \% , 2 . 7 : 1 ~ r r )}\end{array}$ & $\begin{array}{c}\mathrm{PhBr}(3.0 \text { equiv.) } \\
\mathrm{NiCl}_{2} \text { (DME) (5 mol\%) } \\
\mathrm{NaO}^{t} \mathrm{Bu}(3.0 \text { equiv.) } \\
\mathrm{B}_{2} \mathrm{pin}_{2} \text { (4.0 equiv.) } \\
\mathrm{THF}: \mathrm{DMA}(9: 1), \mathrm{rt} \\
\mathbf{6 7 \%}, \mathbf{2 0 : 1} \boldsymbol{r r} \\
\mathbf{( 9 9 \% , 2 . 7 : 1 ~ r r )}\end{array}$ \\
\hline
\end{tabular}




\section{Characterization Data:}

In ${ }^{13} \mathrm{C}$ NMR spectra, signals of carbons directly bonded to boron were not detected because of quadrupolar relaxation.

tert-Butyl 1-phenyl-2-(4,4,5,5-tetramethyl-1,3,2-dioxaborolan-2-yl)-7-azaspiro[3.5]nonane-

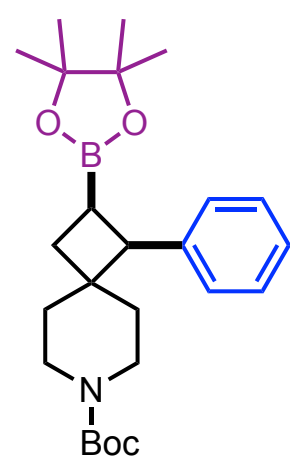

7-carboxylate (5): The title compound was prepared according to General Procedure A. Purification by silica-gel column chromatography (Gradient: hexane to 12\% EtOAc:hexanes) yields 5 as colorless oil.

Average over 2 runs: $67 \mathrm{mg}$ (78\%), >20:1 dr, >20:1 rr; IR (neat): 1692 (s), 1143 (s), $754(\mathrm{w}) \mathrm{cm}^{-1}$; ${ }^{1} \mathbf{H}$ NMR (500 $\left.\mathbf{M H z}, \mathbf{C D C l}_{3}\right): \delta 7.29(\mathrm{~d}, J=7.1 \mathrm{~Hz}$, 2H), $7.27-7.22(\mathrm{~m}, 2 \mathrm{H}), 7.16(\mathrm{t}, J=7.1 \mathrm{~Hz}, 1 \mathrm{H}), 3.58-3.57(\mathrm{~m}, 1 \mathrm{H}), 3.33-$ $3.25(\mathrm{~m}, 2 \mathrm{H}), 3.13-3.08(\mathrm{~m}, 1 \mathrm{H}), 2.93-2.88(\mathrm{~m}, 1 \mathrm{H}), 2.50(\mathrm{q}, J=10.2 \mathrm{~Hz}$, 1H), $2.20(\mathrm{t}, J=11.1 \mathrm{~Hz}, 1 \mathrm{H}), 1.96-1.91(\mathrm{~m}, 1 \mathrm{H}), 1.83-1.76(\mathrm{~m}, 2 \mathrm{H}), 1.40(\mathrm{~s}, 9 \mathrm{H}), 1.31-1.24$ $(\mathrm{m}, 1 \mathrm{H}), 1.17(\mathrm{~s}, 6 \mathrm{H}), 1.12-1.06(\mathrm{~m}, 1 \mathrm{H}), 1.01$ (s, 6H); ${ }^{13} \mathbf{C}$ NMR (126 MHz, CDCl $): \delta 155.0$, $141.7,129.2,127.9,126.2,83.4,79.2,52.0,41.6,38.1,33.6,31.7,28.6,25.2,24.9$; HRMS (ESI+): Calculated for $\mathrm{C}_{25} \mathrm{H}_{38} \mathrm{O}_{4} \mathrm{NBNa}[\mathrm{M}+\mathrm{Na}]+:$ 450.2786, Found: 450.2784 .

tert-Butyl 1-(6-methoxypyridin-3-yl)-2-(4,4,5,5-tetramethyl-1,3,2-dioxaborolan-2-yl)-7-

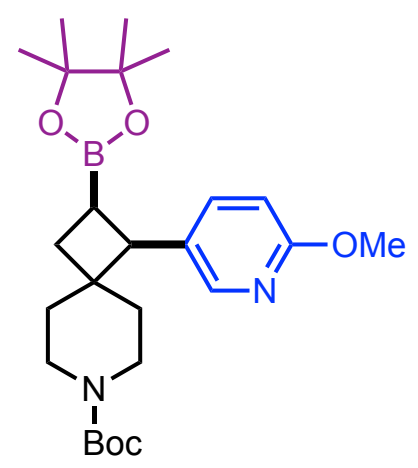
azaspiro[3.5]nonane-7-carboxylate (8): The title compound was prepared according to General Procedure A. Purification by silica-gel column chromatography (Gradient: hexane to 20\% EtOAc:hexanes) yields 8 as colorless oil.

Yield: $50 \mathrm{mg}(54 \%),>20: 1 \mathrm{rr},>20: 1 d r$; IR (neat): 1689 (s), 1142 (s) $\mathrm{cm}^{-1} ;{ }^{1}$ H NMR (400 MHz, $\left.\mathbf{C D C l}_{3}\right): \delta 8.01(\mathrm{~s}, 1 \mathrm{H}), 7.60(\mathrm{dd}, J=8.6$, $2.5 \mathrm{~Hz}, 1 \mathrm{H}), 6.65(\mathrm{~d}, J=8.5 \mathrm{~Hz}, 1 \mathrm{H}), 3.88(\mathrm{~s}, 3 \mathrm{H}), 3.56(\mathrm{~s}, 1 \mathrm{H}), 3.26-$ $3.19(\mathrm{~m}, 2 \mathrm{H}), 3.15-3.12(\mathrm{~m}, 1 \mathrm{H}), 2.93-2.87(\mathrm{~m}, 1 \mathrm{H}), 2.49(\mathrm{q}, J=10.2 \mathrm{~Hz}, 1 \mathrm{H}), 2.13(\mathrm{t}, J=11.0$ $\mathrm{Hz}, 1 \mathrm{H}), 1.92(\mathrm{t}, J=10.5 \mathrm{~Hz}, 1 \mathrm{H}), 1.81-1.69(\mathrm{~m}, 2 \mathrm{H}), 1.38(\mathrm{~s}, 9 \mathrm{H}), 1.31-1.28(\mathrm{~m}, 1 \mathrm{H}), 1.15(\mathrm{~s}$, 6H), $1.08-1.04(\mathrm{~m}, 1 \mathrm{H}), 1.01(\mathrm{~s}, 6 \mathrm{H}) ;{ }^{13} \mathbf{C}$ NMR (126 MHz, CDCl $): \delta 162.9,155.0,147.0$, 139.4, 129.7, 109.7, 83.5, 79.3, 53.4, 48.3, 41.6, 37.9, 33.6, 31.6, 28.5, 25.2, 24.8; HRMS (ESI+): Calculated for $\mathrm{C}_{25} \mathrm{H}_{39} \mathrm{O}_{5} \mathrm{~N}_{2} \mathrm{BNa}[\mathrm{M}+\mathrm{Na}]+:$ 481.2844, Found: 481.2852. 
tert-Butyl

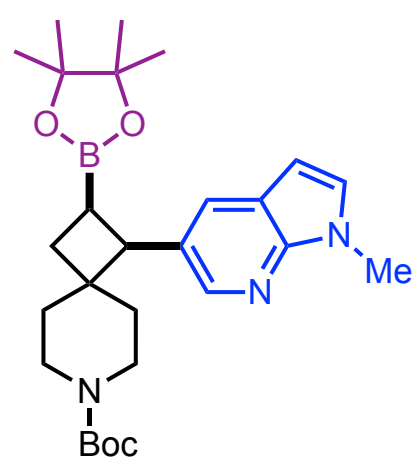

1-(1-methyl-1H-pyrrolo[2,3-b]pyridin-5-yl)-2-(4,4,5,5-tetramethyl-1,3,2dioxaborolan-2-yl)-7-azaspiro[3.5]nonane-7-carboxylate (9): The title compound was prepared according to General Procedure A. Purification by silica-gel column chromatography (Gradient: hexane to $60 \%$ EtOAc:hexanes) yields 9 as colorless oil.

Yield: $75 \mathrm{mg}$ (78\%), >20:1 rr, >20:1 dr; IR (neat): 1688 (s), 1143 (s),

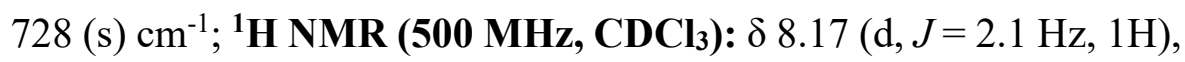
$7.95(\mathrm{~d}, J=2.1 \mathrm{~Hz}, 1 \mathrm{H}), 7.12(\mathrm{~d}, J=3.3 \mathrm{~Hz}, 1 \mathrm{H}), 6.35$ (d, $J=3.4 \mathrm{~Hz}$,

$1 \mathrm{H}), 3.84(\mathrm{~s}, 3 \mathrm{H}), 3.59$ (s, 1H), $3.43(\mathrm{dd}, J=10.5,2.1 \mathrm{~Hz}, 1 \mathrm{H}), 3.29-3.24(\mathrm{~m}, 1 \mathrm{H}), 3.09-3.04$ (m, 1H), $2.90-2.85(\mathrm{~m}, 1 \mathrm{H}), 2.57$ (q, $J=10.2 \mathrm{~Hz}, 1 \mathrm{H}), 2.24(\mathrm{t}, J=11.1 \mathrm{~Hz}, 1 \mathrm{H}), 1.99-1.94$ (m, $1 \mathrm{H}), 1.85-1.76(\mathrm{~m}, 2 \mathrm{H}), 1.37(\mathrm{~s}, 9 \mathrm{H}), 1.29-1.23(\mathrm{~m}, 1 \mathrm{H}), 1.14(\mathrm{~s}, 6 \mathrm{H}), 1.07-1.01(\mathrm{~m}, 1 \mathrm{H}), 0.95$ (s, 6H); ${ }^{13}$ C NMR (126 MHz, CDCl $): \delta$ 155.0, 147.0, 144.3, 129.4, 128.6, 128.4, 119.9, 98.9, 83.4, 79.2, 49.3, 41.8, 38.0, 33.7, 31.7, 31.4, 28.5, 25.1, 24.8; HRMS (ESI+): Calculated for $\mathrm{C}_{27} \mathrm{H}_{41} \mathrm{O}_{4} \mathrm{~N}_{3} \mathrm{~B}[\mathrm{M}+\mathrm{H}]+:$ 482.3185, Found: 482.3179.

tert-Butyl 2-hydroxy-1-(6-methoxypyridin-2-yl)-7-azaspiro[3.5]nonane-7-carboxylate (10):

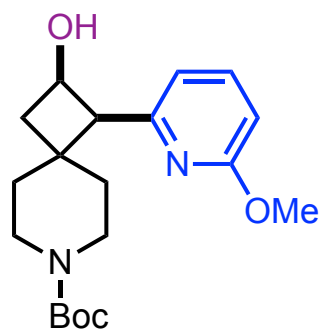

The title compound was prepared according to General Procedure A. Oxidation was performed using $\mathrm{H}_{2} \mathrm{O}_{2} / 2 \mathrm{M} \mathrm{NaOH}$ (aq.). Purification by silicagel column chromatography (Gradient: hexane to 30\% EtOAc:hexanes) yields $\mathbf{1 0}$ as colorless oil.

Average over 2 runs: $68 \mathrm{mg}(98 \%),>20: 1 \mathrm{rr},>20: 1 d r$; IR (neat): 1690 (s),

1577 (s), 1152 (s) cm ${ }^{-1} ;{ }^{1} \mathbf{H}$ NMR (400 MHz, $\left.\mathbf{C D C l}_{3}\right): \delta 7.51$ (t, $\left.J=7.5 \mathrm{~Hz}, 1 \mathrm{H}\right), 6.69$ (d, $J=7.2$ $\mathrm{Hz}, 1 \mathrm{H}), 6.61(\mathrm{~d}, J=8.3 \mathrm{~Hz}, 1 \mathrm{H}), 5.86(\mathrm{~s}, 1 \mathrm{H}), 4.63(\mathrm{q}, J=7.5 \mathrm{~Hz}, 1 \mathrm{H}), 3.92(\mathrm{~s}, 3 \mathrm{H}), 3.63(\mathrm{~d}, J=$ $13.4 \mathrm{~Hz}, 1 \mathrm{H}), 3.44$ (dd, $J=7.6,2.5 \mathrm{~Hz}, 1 \mathrm{H}), 3.29$ (d, $J=13.0 \mathrm{~Hz}, 1 \mathrm{H}), 3.18-3.11(\mathrm{~m}, 1 \mathrm{H}), 2.92$ $-2.86(\mathrm{~m}, 1 \mathrm{H}), 2.39-2.35(\mathrm{~m}, 1 \mathrm{H}), 2.12(\mathrm{dd}, J=11.7,7.2 \mathrm{~Hz}, 1 \mathrm{H}), 1.72-1.62(\mathrm{~m}, 2 \mathrm{H}), 1.39(\mathrm{~s}$,

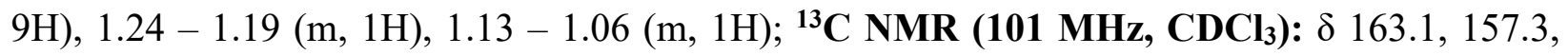
154.9, 139.5, 118.6, 108.6, 79.4, 65.5, 54.1, 53.7, 42.5, 38.3, 37.0, 33.8, 28.5; HRMS (ESI+): Calculated for $\mathrm{C}_{19} \mathrm{H}_{29} \mathrm{O}_{4} \mathrm{~N}_{2}[\mathrm{M}+\mathrm{H}]+:$ 349.2122, Found: 349.2125 . 
tert-Butyl 1-(6-methoxy-2-methylpyridin-3-yl)-2-(4,4,5,5-tetramethyl-1,3,2-dioxaborolan-2-

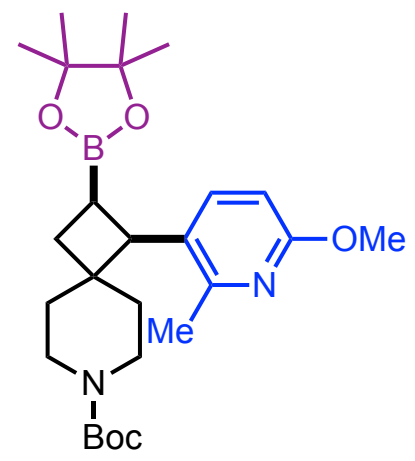

yl)-7-azaspiro[3.5]nonane-7-carboxylate (11): The title compound was prepared according to General Procedure A. Purification by silicagel column chromatography (Gradient: hexane to $22 \%$ EtOAc:hexanes) yields 11 as colorless oil.

Yield: $53 \mathrm{mg}$ (56\%), 20:1 rr, >20:1 dr; IR (neat): 1689 (s), 1305 (s),

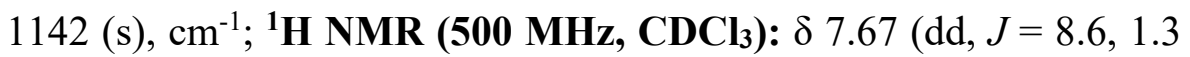
$\mathrm{Hz}, 1 \mathrm{H}), 6.53(\mathrm{~d}, J=8.4 \mathrm{~Hz}, 1 \mathrm{H}), 3.87(\mathrm{~s}, 3 \mathrm{H}), 3.73-3.70(\mathrm{~m}, 1 \mathrm{H})$, $3.51(\mathrm{~d}, J=10.3 \mathrm{~Hz}, 1 \mathrm{H}), 3.27(\mathrm{~d}, J=13.4 \mathrm{~Hz}, 1 \mathrm{H}), 3.12(\mathrm{t}, J=11.8 \mathrm{~Hz}, 1 \mathrm{H}), 2.78$ (t, $J=10.3 \mathrm{~Hz}$, 1H), 2.51 (q, $J=10.3 \mathrm{~Hz}, 1 \mathrm{H}), 2.37$ (s, 3H), $2.10(\mathrm{t}, J=11.4 \mathrm{~Hz}, 1 \mathrm{H}), 1.98-1.89(\mathrm{~m}, 2 \mathrm{H}), 1.76-$ $1.71(\mathrm{~m}, 1 \mathrm{H}), 1.40(\mathrm{~s}, 9 \mathrm{H}), 1.18(\mathrm{~s}, 6 \mathrm{H}), 1.13-1.10(\mathrm{~m}, 1 \mathrm{H}), 1.06(\mathrm{~s}, 6 \mathrm{H}), 0.96-0.87(\mathrm{~m}, 1 \mathrm{H})$; ${ }^{13}$ C NMR (126 MHz, CDCl $): \delta 161.5,155.0,154.1,138.6,127.2,106.6,83.5,79.3,53.3,45.9$, 41.9, 37.8, 34.1, 31.3, 28.5, 25.2, 24.9, 23.1; HRMS (ESI+): Calculated for $\mathrm{C}_{26} \mathrm{H}_{42} \mathrm{O}_{5} \mathrm{~N}_{2} \mathrm{~B}$ $[\mathrm{M}+\mathrm{H}]+:$ 473.3181, Found: 473.3181 .

tert-Butyl 1-(6-(piperidin-1-yl)pyridin-3-yl)-2-(4,4,5,5-tetramethyl-1,3,2-dioxaborolan-2-yl)-

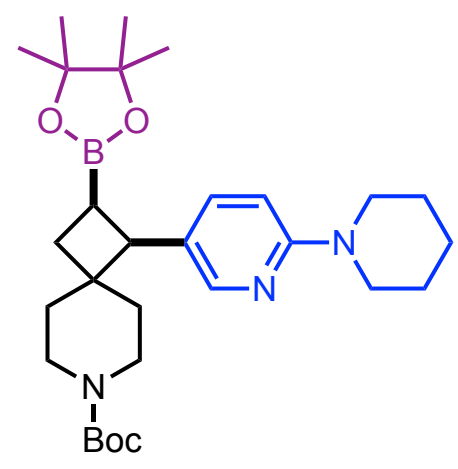

7-azaspiro[3.5]nonane-7-carboxylate (12): The title compound was prepared according to General Procedure A. Purification by silica-gel column chromatography (Gradient: hexane to $60 \%$ EtOAc:hexanes) yields 12 as colorless oil.

Yield: $66 \mathrm{mg}(65 \%),>20: 1 \mathrm{rr},>20: 1 d r$; IR (neat): $1687(\mathrm{w}), 1142$ (s), 730 (s) $\mathrm{cm}^{-1} ;{ }^{1} \mathbf{H}$ NMR (500 MHz, $\left.\mathbf{C D C l}_{3}\right): \delta 8.04$ (d, $J=2.6$ $\mathrm{Hz}, 1 \mathrm{H}), 7.48(\mathrm{dd}, J=8.8,2.6 \mathrm{~Hz}, 1 \mathrm{H}), 6.56(\mathrm{~d}, J=8.7 \mathrm{~Hz}, 1 \mathrm{H})$, $3.54-3.52(\mathrm{~m}, 1 \mathrm{H}), 3.46-3.42(\mathrm{~m}, 4 \mathrm{H}), 3.26-3.21(\mathrm{~m}, 1 \mathrm{H}), 3.18-3.10(\mathrm{~m}, 2 \mathrm{H}), 2.94-2.89$ (m, 1H), 2.45 (q, $J=10.1 \mathrm{~Hz}, 1 \mathrm{H}), 2.11(\mathrm{t}, J=11.0 \mathrm{~Hz}, 1 \mathrm{H}), 1.89$ (d, $J=9.6 \mathrm{~Hz}, 1 \mathrm{H}), 1.77-1.68$ $(\mathrm{m}, 2 \mathrm{H}), 1.61(\mathrm{~s}, 6 \mathrm{H}), 1.38(\mathrm{~s}, 9 \mathrm{H}), 1.30-1.19(\mathrm{~m}, 1 \mathrm{H}), 1.16(\mathrm{~s}, 6 \mathrm{H}), 1.13-1.08(\mathrm{~m}, 1 \mathrm{H}), 1.02(\mathrm{~s}$, 6H); ${ }^{13} \mathbf{C}$ NMR (126 MHz, $\left.\mathbf{C D C l}_{3}\right): \delta$ 158.8, 155.0, 148.3, 138.1, 125.4, 106.3, 83.4, 79.2, 48.3, 46.8, 41.7, 37.9, 33.6, 31.7, 28.5, 25.5, 25.2, 24.8, 24.8; HRMS (ESI+): Calculated for $\mathrm{C}_{29} \mathrm{H}_{47} \mathrm{O}_{4} \mathrm{~N}_{3} \mathrm{~B}[\mathrm{M}+\mathrm{H}]+:$ 512.3654, Found: 512.3660 . 
tert-Butyl 1-(2-morpholinopyrimidin-5-yl)-2-(4,4,5,5-tetramethyl-1,3,2-dioxaborolan-2-yl)-

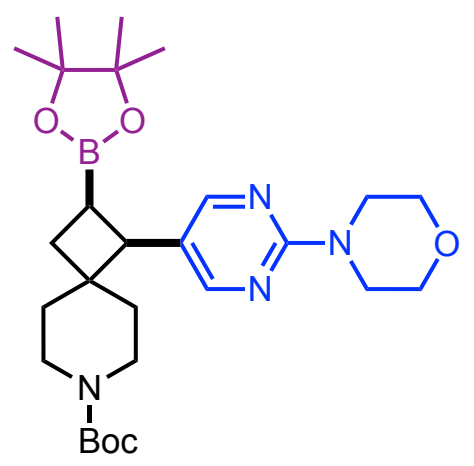
7-azaspiro[3.5]nonane-7-carboxylate (13): The title compound was prepared according to General Procedure A. Purification by silica-gel column chromatography (Gradient: hexane to $30 \%$ EtOAc:hexanes) yields 13 as colorless oil.

Average over 2 runs: $40 \mathrm{mg}(40 \%), 20: 1 r r,>20: 1 d r$; IR (neat): 1693 (s), 1599 (s), 1143 (s), cm ${ }^{-1} ;{ }^{1}$ H NMR (500 MHz, CDCl 3 ): $\delta$ $8.29(\mathrm{~s}, 2 \mathrm{H}), 3.74(\mathrm{~s}, 8 \mathrm{H}), 3.61-3.57(\mathrm{~m}, 1 \mathrm{H}), 3.26-3.22(\mathrm{~m}, 1 \mathrm{H})$, $3.20-3.15(\mathrm{~m}, 1 \mathrm{H}), 3.10(\mathrm{~d}, J=10.5 \mathrm{~Hz}, 1 \mathrm{H}), 2.95-2.89(\mathrm{~m}, 1 \mathrm{H}), 2.47(\mathrm{q}, J=10.1 \mathrm{~Hz}, 1 \mathrm{H})$, $2.10(\mathrm{t}, J=10.9 \mathrm{~Hz}, 1 \mathrm{H}), 1.94(\mathrm{t}, J=10.6 \mathrm{~Hz}, 1 \mathrm{H}), 1.79-1.75(\mathrm{~m}, 1 \mathrm{H}), 1.71-1.66(\mathrm{~m}, 1 \mathrm{H}), 1.39$ (s, 9H), $1.26-1.21(\mathrm{~m}, 1 \mathrm{H}), 1.17(\mathrm{~s}, 6 \mathrm{H}), 1.13-1.09(\mathrm{~m}, 1 \mathrm{H}), 1.06(\mathrm{~s}, 6 \mathrm{H}) ;{ }^{13} \mathrm{C}$ NMR (126 MHz, $\left.\mathbf{C D C l}_{3}\right): \delta 161.0,158.1,155.0,122.9,83.6,79.4,66.9,46.2,44.6,41.7,37.9,33.6,31.6,28.5$, 25.2, 24.8; HRMS (ESI+): Calculated for $\mathrm{C}_{27} \mathrm{H}_{44} \mathrm{O}_{5} \mathrm{~N}_{4} \mathrm{~B}$ [M+H]+: 515.3399, Found: 515.3394.

tert-Butyl 2-(4,4,5,5-tetramethyl-1,3,2-dioxaborolan-2-yl)-1-(o-tolyl)-7-azaspiro[3.5]nonane-

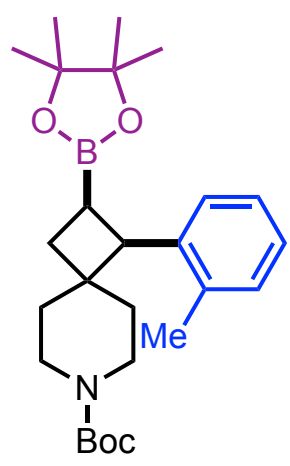

7-carboxylate (14): The title compound was prepared according to General Procedure A. Purification by silica-gel column chromatography (Gradient: hexane to $13 \%$ EtOAc:hexanes) yields 14 as colorless oil.

Yield: $62 \mathrm{mg}$ (70\%), >20:1 dr,>20:1 rr; IR (neat): 1692 (s), 1143 (s), 751 (s) $\mathrm{cm}^{-1} ;{ }^{1} \mathbf{H}$ NMR (400 MHz, $\left.\mathbf{C D C l}_{3}\right): \delta 7.45(\mathrm{dd}, J=7.6,1.3 \mathrm{~Hz}, 1 \mathrm{H}), 7.13(\mathrm{td}$, $J=7.2,2.0 \mathrm{~Hz}, 1 \mathrm{H}), 7.10-7.02(\mathrm{~m}, 2 \mathrm{H}), 3.77$ (s, 1H), 3.60 (dd, $J=10.3,2.2$ $\mathrm{Hz}, 1 \mathrm{H}), 3.31(\mathrm{~d}, J=12.9 \mathrm{~Hz}, 1 \mathrm{H}), 3.13-3.06(\mathrm{~m}, 1 \mathrm{H}), 2.76-2.69(\mathrm{~m}, 1 \mathrm{H})$, $2.51(\mathrm{q}, J=10.0 \mathrm{~Hz}, 1 \mathrm{H}), 2.22(\mathrm{~s}, 3 \mathrm{H}), 2.16(\mathrm{t}, J=11.4 \mathrm{~Hz}, 1 \mathrm{H}), 2.02-1.93(\mathrm{~m}, 2 \mathrm{H}), 1.79-1.72$ (m, 1H), 1.40 (s, 9H), $1.21(\mathrm{~s}, 6 \mathrm{H}), 1.11-1.09(\mathrm{~m}, 1 \mathrm{H}), 1.07(\mathrm{~s}, 6 \mathrm{H}), 0.94-0.87(\mathrm{~m}, 1 \mathrm{H}) ;{ }^{13} \mathrm{C}$ NMR (126 MHz, $\left.\mathbf{C D C l}_{3}\right): \delta$ 155.0, 139.7, 136.3, 130.3, 127.8, 125.9, 125.3, 83.4, 79.2, 47.0, 41.8, 37.8, 34.1, 31.2, 28.5, 25.1, 24.9, 20.8; HRMS (ESI+): Calculated for $\mathrm{C}_{26} \mathrm{H}_{40} \mathrm{O}_{4} \mathrm{NBNa}$ $[\mathrm{M}+\mathrm{Na}]^{+}:$464.2943, Found: 464.2945. 
tert-Butyl 1-(2-methoxypyridin-4-yl)-2-(4,4,5,5-tetramethyl-1,3,2-dioxaborolan-2-yl)-7-

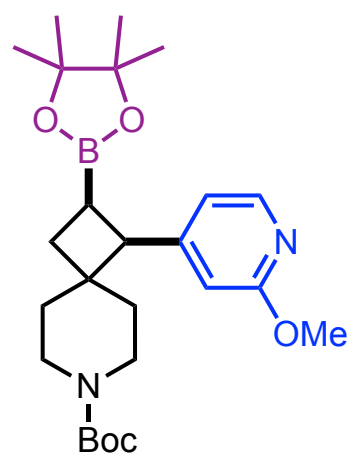
azaspiro[3.5]nonane-7-carboxylate (15): The title compound was prepared according to General Procedure A. Purification by silica-gel column chromatography (Gradient: hexane to $20 \%$ EtOAc:hexanes) yields 15 as colorless oil.

Average over 2 runs: $69 \mathrm{mg}(76 \%),>20: 1 \mathrm{rr},>20: 1 d r$; IR (neat): 1693 (s), 1610 (w), 1143 (s), cm ${ }^{-1}$; ${ }^{1}$ H NMR (400 MHz, CDCl $): \delta 8.00$ (d, $J=$ $5.3 \mathrm{~Hz}, 1 \mathrm{H}), 6.77(\mathrm{~d}, J=5.7 \mathrm{~Hz}, 1 \mathrm{H}), 6.65(\mathrm{~s}, 1 \mathrm{H}), 3.89$ (s, 3H), 3.57 (s, $1 \mathrm{H}), 3.24-3.14(\mathrm{~m}, 3 \mathrm{H}), 2.91-2.84(\mathrm{~m}, 1 \mathrm{H}), 2.44(\mathrm{q}, J=10.1 \mathrm{~Hz}, 1 \mathrm{H}), 2.14(\mathrm{t}, J=11.0 \mathrm{~Hz}, 1 \mathrm{H})$, $1.96-1.90(\mathrm{~m}, 1 \mathrm{H}), 1.82-1.70(\mathrm{~m}, 2 \mathrm{H}), 1.38(\mathrm{~s}, 9 \mathrm{H}), 1.31-1.22(\mathrm{~m}, 1 \mathrm{H}), 1.17(\mathrm{~s}, 6 \mathrm{H}), 1.11-$ 1.07 (m, 1H), 1.04 (s, 6H); ${ }^{13} \mathbf{C}$ NMR (101 MHz, $\left.\mathbf{C D C l}_{3}\right): \delta 164.3,154.9,153.5,146.2,118.1$, $110.8,83.6,79.3,53.4,51.1,41.6,38.0,33.4,31.6,28.5,25.1,24.8$.

HRMS (ESI+): Calculated for $\mathrm{C}_{25} \mathrm{H}_{40} \mathrm{O}_{5} \mathrm{~N}_{2} \mathrm{~B}$ [M+H]+: 459.3025, Found: 459.3027.

tert-Butyl 1-(benzo[d][1,3]dioxol-5-yl)-2-(4,4,5,5-tetramethyl-1,3,2-dioxaborolan-2-yl)-7-

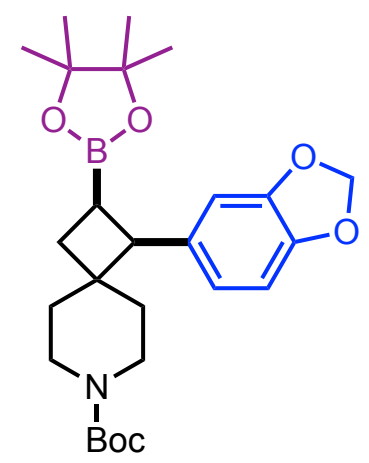
azaspiro[3.5]nonane-7-carboxylate (16): The title compound was prepared according to General Procedure A. Purification by silica-gel column chromatography (Gradient: hexane to 14\% EtOAc:hexanes) yields 16 as colorless oil.

Yield: $59 \mathrm{mg}(63 \%),>20: 1 \mathrm{rr},>20: 1 d r$; IR (neat): 1688 (s), 1241 (s), $1143(\mathrm{~s}), \mathrm{cm}^{-1} ;{ }^{1} \mathbf{H}$ NMR (500 MHz, CDCl$): \delta 6.84(\mathrm{~d}, J=1.6 \mathrm{~Hz}, 1 \mathrm{H})$, $6.69-6.67(\mathrm{~m}, 2 \mathrm{H}), 5.89(\mathrm{dd}, J=7.7,1.5 \mathrm{~Hz}, 2 \mathrm{H}), 3.57-3.54(\mathrm{~m}, 1 \mathrm{H})$, $3.27-3.22(\mathrm{~m}, 2 \mathrm{H}), 3.17-3.12(\mathrm{~m}, 1 \mathrm{H}), 2.95-2.89(\mathrm{~m}, 1 \mathrm{H}), 2.46(\mathrm{q}, J=10.2 \mathrm{~Hz}, 1 \mathrm{H}), 2.13(\mathrm{t}$, $J=11.1 \mathrm{~Hz}, 1 \mathrm{H}), 1.92-1.86(\mathrm{~m}, 1 \mathrm{H}), 1.79-1.70$ (m, 2H), 1.39 (s, 9H), $1.32-1.27$ (m, 1H), 1.18 (s, 6H), 1.15 - 1.09 (m, 1H), 1.05 (s, 6H); ${ }^{13} \mathbf{C}$ NMR (126 MHz, CDCl $): \delta 155.0,147.2,145.8$, 135.6, 122.2, 109.5, 107.7, 100.9, 83.4, 79.2, 51.8, 41.7, 38.1, 33.5, 31.6, 28.6, 25.2, 24.9; HRMS (ESI+): Calculated for $\mathrm{C}_{26} \mathrm{H}_{38} \mathrm{O}_{6} \mathrm{NBNa}[\mathrm{M}+\mathrm{Na}]+:$ 494.2684, Found: 494.2684 . 
tert-Butyl 1-(4-(ethoxycarbonyl)phenyl)-2-(4,4,5,5-tetramethyl-1,3,2-dioxaborolan-2-yl)-7-

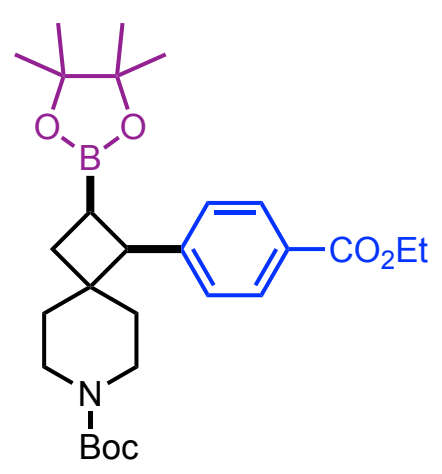
azaspiro[3.5]nonane-7-carboxylate (17): The title compound was prepared according to General Procedure A. Purification by silica-gel column chromatography (Gradient: hexane to 17\% EtOAc:hexanes) yields 17 as colorless oil.

Yield: $80 \mathrm{mg}(80 \%),>20: 1 d r,>20: 1 \mathrm{rr}$; IR (neat): 1715 (s), 1690 (s), 1142 (s), $753(\mathrm{w}) \mathrm{cm}^{-1}$; ${ }^{1} \mathbf{H}$ NMR (400 MHz, $\left.\mathbf{C D C l}_{3}\right): \delta 7.92$ (d, $J=$ $8.4 \mathrm{~Hz}, 2 \mathrm{H}), 7.34(\mathrm{~d}, J=8.4 \mathrm{~Hz}, 2 \mathrm{H}), 4.34(\mathrm{qd}, J=7.1,1.4 \mathrm{~Hz}, 2 \mathrm{H})$, $3.57(\mathrm{~s}, 1 \mathrm{H}), 3.37(\mathrm{~d}, J=10.8 \mathrm{~Hz}, 1 \mathrm{H}), 3.28-3.21(\mathrm{~m}, 1 \mathrm{H}), 3.12-3.06(\mathrm{~m}, 1 \mathrm{H}), 2.90-2.84(\mathrm{~m}$, $1 \mathrm{H}), 2.50(\mathrm{q}, J=10.2 \mathrm{~Hz}, 1 \mathrm{H}), 2.19(\mathrm{t}, J=11.1 \mathrm{~Hz}, 1 \mathrm{H}), 1.98-1.92(\mathrm{~m}, 1 \mathrm{H}), 1.85-1.73(\mathrm{~m}, 2 \mathrm{H})$, $1.42-1.35(\mathrm{~m}, 12 \mathrm{H}), 1.24-1.21(\mathrm{~m}, 1 \mathrm{H}), 1.16(\mathrm{~s}, 6 \mathrm{H}), 1.01(\mathrm{~s}, 7 \mathrm{H}) ;{ }^{13} \mathrm{C}$ NMR (101 MHz, $\left.\mathbf{C D C l}_{3}\right): \delta 166.7,154.9,147.2,129.2,129.0,128.5,83.5,79.3,60.9,52.0,41.9,38.1,33.5,31.6$, 28.5, 25.1, 24.9, 14.5; HRMS (ESI+): Calculated for $\mathrm{C}_{28} \mathrm{H}_{42} \mathrm{O}_{6} \mathrm{NBNa}[\mathrm{M}+\mathrm{Na}]+$ : 522.2997, Found: 522.3003 .

tert-Butyl 1-(benzofuran-5-yl)-2-(4,4,5,5-tetramethyl-1,3,2-dioxaborolan-2-yl)-7-azaspiro

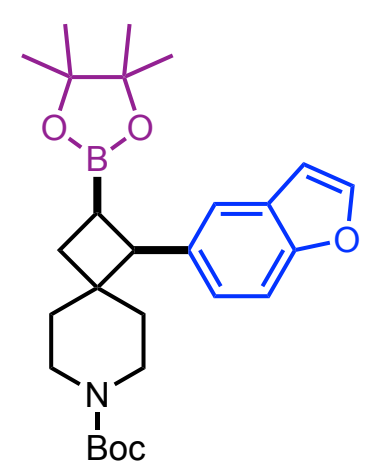
[3.5]nonane-7-carboxylate (18): The title compound was prepared according to General Procedure A. Purification by silica-gel column chromatography (Gradient: hexane to 11\% EtOAc:hexanes) yields $\mathbf{1 8}$ as colorless oil.

Average yield over 2 runs: $69 \mathrm{mg}$ (74\%), >20:1 dr, >20:1 rr; IR (neat): 1688 (s), 1143 (s), 737 (w) cm ${ }^{-1} ;{ }^{1} \mathbf{H}$ NMR (400 MHz, CDCl 3 ): $\delta 7.55$ $(\mathrm{dd}, J=9.1,2.0 \mathrm{~Hz}, 2 \mathrm{H}), 7.36(\mathrm{~d}, J=8.4 \mathrm{~Hz}, 1 \mathrm{H}), 7.20(\mathrm{dd}, J=8.5,1.9$ $\mathrm{Hz}, 1 \mathrm{H}), 6.68(\mathrm{~d}, J=2.2 \mathrm{~Hz}, 1 \mathrm{H}), 3.58(\mathrm{~s}, 1 \mathrm{H}), 3.44-3.41(\mathrm{~m}, 1 \mathrm{H}), 3.33-3.26(\mathrm{~m}, 1 \mathrm{H}), 3.10-$ $3.04(\mathrm{~m}, 1 \mathrm{H}), 2.93-2.87(\mathrm{~m}, 1 \mathrm{H}), 2.55(\mathrm{q}, J=10.2 \mathrm{~Hz}, 1 \mathrm{H}), 2.25(\mathrm{t}, J=11.1 \mathrm{~Hz}, 1 \mathrm{H}), 1.97-1.92$ $(\mathrm{m}, 1 \mathrm{H}), 1.83-1.79(\mathrm{~m}, 2 \mathrm{H}), 1.38(\mathrm{~s}, 9 \mathrm{H}), 1.31-1.22(\mathrm{~m}, 1 \mathrm{H}), 1.15(\mathrm{~s}, 6 \mathrm{H}), 1.12-1.05(\mathrm{~m}, 1 \mathrm{H})$, 0.96 (s, 6H); ${ }^{13} \mathbf{C}$ NMR (101 MHz, $\left.\mathbf{C D C l}_{3}\right): \delta$ 154.9, 153.8, 145.2, 136.2, 127.1, 125.8, 121.1, 110.5, 106.5, 83.3, 79.1, 51.9, 41.7, 38.2, 33.6, 31.7, 28.5, 25.1, 24.9; HRMS (ESI+): Calculated for $\mathrm{C}_{27} \mathrm{H}_{38} \mathrm{O}_{5} \mathrm{NBNa}[\mathrm{M}+\mathrm{Na}]+:$ 490.2735, Found: 490.2734. 
tert-Butyl 5-phenyl-6-(4,4,5,5-tetramethyl-1,3,2-dioxaborolan-2-yl)-2-azaspiro[3.3]heptane-

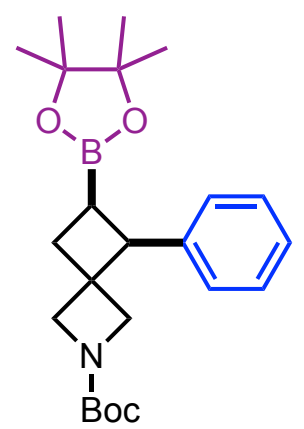

2-carboxylate (19): The title compound was prepared according to General Procedure A. Purification by silica-gel column chromatography (Gradient: hexane to 20\% EtOAc:hexanes) yields 19 as colorless oil.

Yield: $65 \mathrm{mg}$ (81\%), >20:1 rr, >20:1 dr; IR (neat): 1704 (s), 1141 (s), 1096

(s) $\mathrm{cm}^{-1} ;{ }^{1} \mathbf{H}$ NMR (400 MHz, $\left.\mathbf{C D C l}_{3}\right): \delta 7.29-7.25(\mathrm{~m}, 2 \mathrm{H}), 7.19-7.15(\mathrm{~m}$, $3 \mathrm{H}), 4.06(\mathrm{~d}, J=8.8 \mathrm{~Hz}, 1 \mathrm{H}), 4.00(\mathrm{~d}, J=8.8 \mathrm{~Hz}, 1 \mathrm{H}), 3.82(\mathrm{~d}, J=11.9 \mathrm{~Hz}$, $1 \mathrm{H}), 3.72(\mathrm{~d}, J=9.5 \mathrm{~Hz}, 1 \mathrm{H}), 3.65(\mathrm{dd}, J=8.5,2.9 \mathrm{~Hz}, 1 \mathrm{H}), 2.48-2.42(\mathrm{~m}$,

1H), $2.33-2.27(\mathrm{~m}, 2 \mathrm{H}), 1.40$ (s, 9H), 1.00 (s, 6H), $0.96(\mathrm{~s}, 6 \mathrm{H}) ;{ }^{13} \mathbf{C}$ NMR (126 MHz, CDCl 3$):$ $\delta 156.5,141.6,128.4,127.6,126.4,83.3,79.2,62.6,50.2,41.3,33.4,28.5,24.9,24.7$; HRMS (ESI+): Calculated for $\mathrm{C}_{23} \mathrm{H}_{34} \mathrm{O}_{4} \mathrm{NBNa}[\mathrm{M}+\mathrm{Na}]+:$ 422.2473, Found: 422.2475 .

\section{2-Benzyl-5-phenyl-6-(4,4,5,5-tetramethyl-1,3,2-dioxaborolan-2-yl)-2-azaspiro[3.3]heptane}

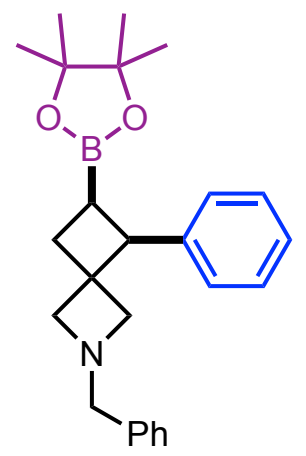

(20): The title compound was prepared according to General Procedure A. Purification by silica-gel column chromatography (Gradient: hexane to 80\% EtOAc:hexanes) yields $\mathbf{2 0}$ as colorless oil.

Yield: $61 \mathrm{mg}$ (78 \%), >20:1 rr, >20:1 dr; IR (neat): 1379 (s), 1320 (w), 1143

(s) $\mathrm{cm}^{-1} ;{ }^{1} \mathbf{H}$ NMR (400 MHz, $\left.\mathbf{C D C l}_{3}\right): \delta 7.30-7.15(\mathrm{~m}, 10 \mathrm{H}), 3.57-3.54$ (m, 4H), $3.36-3.33(\mathrm{~m}, 1 \mathrm{H}), 3.24(\mathrm{~d}, J=8.2 \mathrm{~Hz}, 1 \mathrm{H}), 2.98(\mathrm{~d}, J=8.1 \mathrm{~Hz}$, $1 \mathrm{H}), 2.68-2.60(\mathrm{~m}, 1 \mathrm{H}), 2.40-2.32(\mathrm{~m}, 2 \mathrm{H}), 1.00(\mathrm{~s}, 6 \mathrm{H}), 0.92(\mathrm{~s}, 6 \mathrm{H}) ;{ }^{13} \mathrm{C}$

NMR (101 MHz, CDCl $): \delta$ 142.3, 138.4, 128.6, 128.3, 128.2, 126.9, 126.1, 83.1, 68.3, 63.8, 63.1, 51.4, 42.3, 34.0, 24.9, 24.7; HRMS (ESI+): Calculated for $\mathrm{C}_{25} \mathrm{H}_{33} \mathrm{O}_{2} \mathrm{NB}[\mathrm{M}+\mathrm{H}]+$ : 390.2599 , Found: 390.2603 . 
tert-Butyl 1-phenyl-2-(4,4,5,5-tetramethyl-1,3,2-dioxaborolan-2-yl)-9-azadispiro[3.1.56.14]

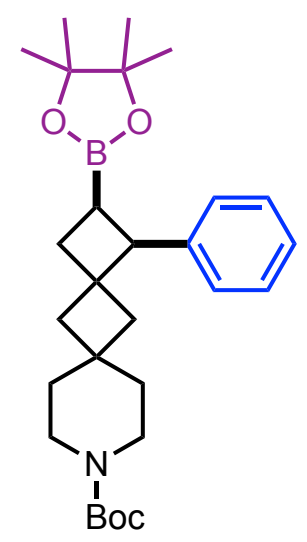
dodecane-9-carboxylate (21): The title compound was prepared according to General Procedure A. Purification by silica-gel column chromatography (Gradient: hexane to 20\% EtOAc:hexanes) yields 21 as colorless oil.

Yield: $81 \mathrm{mg}(87 \%),>20: 1 \mathrm{rr},>20: 1 \mathrm{dr}$; IR (neat): 1691 (s), 1140 (s), 694 (w) $\mathrm{cm}^{-1} ;{ }^{1} \mathbf{H}$ NMR (400 MHz, $\left.\mathbf{C D C l}_{3}\right): \delta 7.25-7.18(\mathrm{~m}, 4 \mathrm{H}), 7.14-7.09$ $(\mathrm{m}, 1 \mathrm{H}), 3.34-3.27(\mathrm{~m}, 2 \mathrm{H}), 3.24-3.16(\mathrm{~m}, 2 \mathrm{H}), 3.11-3.04(\mathrm{~m}, 1 \mathrm{H}), 2.55$ $(\mathrm{t}, J=10.8 \mathrm{~Hz}, 1 \mathrm{H}), 2.43(\mathrm{~d}, J=9.7 \mathrm{~Hz}, 1 \mathrm{H}), 2.14-2.06(\mathrm{~m}, 2 \mathrm{H}), 1.98(\mathrm{~d}, J$ $=11.5 \mathrm{~Hz}, 1 \mathrm{H}), 1.62-1.57(\mathrm{~m}, 1 \mathrm{H}), 1.53-1.43(\mathrm{~m}, 3 \mathrm{H}), 1.39(\mathrm{~s}, 9 \mathrm{H}), 1.31-$ $1.18(\mathrm{~m}, 2 \mathrm{H}), 0.99$ (s, 6H), 0.86 (s, 6H); ${ }^{13} \mathbf{C}$ NMR (101 MHz, CDCl 3$): \delta 155.1,142.9,128.6$, $128.1,126.0,83.1,79.2,54.8,47.7,41.1,40.9,38.2,37.7,32.5,28.6,25.0,24.7$; HRMS (ESI+): Calculated for $\mathrm{C}_{28} \mathrm{H}_{42} \mathrm{O}_{4} \mathrm{NBNa}[\mathrm{M}+\mathrm{Na}]+:$ 490.3099, Found: 490.3103 .

tert-Butyl 1-phenyl-2-(4,4,5,5-tetramethyl-1,3,2-dioxaborolan-2-yl)-6-azaspiro[3.5]nonane-

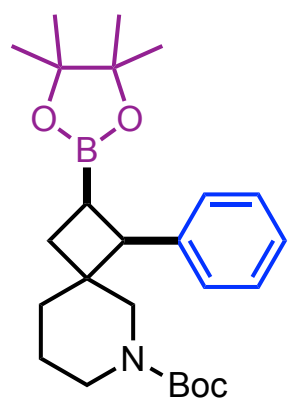
6-carboxylate (23): The title compound was prepared according to General Procedure A. Purification by silica-gel column chromatography (Gradient: hexane to 20\% EtOAc:hexanes) yields 23 as colorless oil.

Yield: $72 \mathrm{mg}(84 \%),>20: 1 \mathrm{rr}, 1: 1 d r$; IR (neat): 1690 (s), 1380 (s), 1145 (s) $\mathrm{cm}^{-1} ;{ }^{1} \mathbf{H}$ NMR (500 MHz, $\left.\mathbf{C D C l}_{3}\right): \delta 7.27-7.21(\mathrm{~m}, 4 \mathrm{H}), 7.16-7.13(\mathrm{~m}$, $1 \mathrm{H}), 3.75-3.57(\mathrm{~m}, 1 \mathrm{H}), 3.43-3.32(\mathrm{~m}, 2 \mathrm{H}), 3.26-3.24(\mathrm{~m}, 1 \mathrm{H}), 3.13-$ $2.95(\mathrm{~m}, 1 \mathrm{H}), 2.62-2.46(\mathrm{~m}, 1 \mathrm{H}), 2.12-2.06(\mathrm{~m}, 1 \mathrm{H}), 1.90-1.86(\mathrm{~m}, 1 \mathrm{H}), 1.49(\mathrm{~s}, 9 \mathrm{H}), 1.30-$ $1.23(\mathrm{~m}, 2 \mathrm{H}), 1.15(\mathrm{~s}, 6 \mathrm{H}), 1.11-1.03(\mathrm{~m}, 2 \mathrm{H}), 0.97(\mathrm{~s}, 6 \mathrm{H}) ;{ }^{13} \mathbf{C}$ NMR (126 MHz, CDCl$): \delta$ 155.3, 141.6, 129.2, 127.8, 126.2, 83.3, 79.4, 54.6, 53.4, 49.6, 44.8, 43.6, 42.7, 32.5, 30.5, 28.6, 25.1, 24.8; HRMS (ESI+): Calculated for $\mathrm{C}_{25} \mathrm{H}_{38} \mathrm{O}_{4} \mathrm{NBNa}[\mathrm{M}+\mathrm{Na}]+:$ 450.2786, Found: 450.2789. 
tert-Butyl 1-phenyl-2-(4,4,5,5-tetramethyl-1,3,2-dioxaborolan-2-yl)-6-azaspiro[3.4]octane-6-

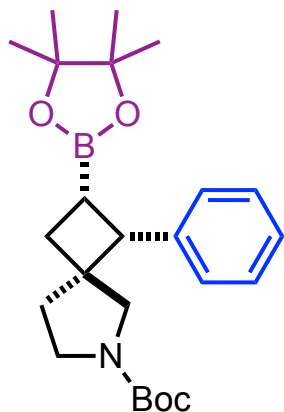

carboxylate (25): The title compound was prepared according to General Procedure A. Purification by silica-gel column chromatography (Gradient: hexane to $20 \%$ EtOAc:hexanes) yields 25 as colorless oil.

Yield: $26 \mathrm{mg}$ (31\%, minor), >20:1 rr, >20:1 $d r$ (99\% NMR yield, 1.7:1 dr); IR (neat): 1697 (s), 1402 (s), 1382 (s), 1143 (w) cm ${ }^{-1}$; 1 H NMR (400 MHz, $\mathrm{CDCl}_{3}$, mixture of rotamers): $\delta 7.31-7.24(\mathrm{~m}, 4 \mathrm{H}), 7.19-7.15(\mathrm{~m}, 1 \mathrm{H})$, $3.50-3.45(\mathrm{~m}, 3 \mathrm{H}), 3.25-2.99(\mathrm{~m}, 2 \mathrm{H}), 2.46(\mathrm{q}, J=9.8 \mathrm{~Hz}, 1 \mathrm{H}), 2.40-2.33(\mathrm{~m}, 1 \mathrm{H}), 2.12-$ $2.04(\mathrm{~m}, 1 \mathrm{H}), 1.67-1.60(\mathrm{~m}, 1 \mathrm{H}), 1.46$ (d, $J=13.5 \mathrm{~Hz}, 9 \mathrm{H}), 1.16$ (s, 6H), 1.01 (s, 6H), $0.98-$ $0.88(\mathrm{~m}, 1 \mathrm{H}) ;{ }^{13} \mathbf{C}$ NMR (126 $\mathbf{M H z}, \mathbf{C D C l}_{3}$, mixture of rotamers): $\delta 155.0,141.9,141.7,128.8$, $128.1,128.1,126.5,126.4,83.4,83.4,79.2,79.1,59.0,58.5,50.0,50.0,49.7,48.8,44.1,44.3$, 33.4, 32.6, 32.2, 32.2, 28.7, 28.7, 25.1, 25.1, 24.8, 24.8; HRMS (ESI+): Calculated for $\mathrm{C}_{24} \mathrm{H}_{36} \mathrm{O}_{4} \mathrm{NBNa}[\mathrm{M}+\mathrm{Na}]+:$ 436.2630, Found: 436.2630 .

tert-Butyl 1-phenyl-2-(4,4,5,5-tetramethyl-1,3,2-dioxaborolan-2-yl)-6-azaspiro[3.4]octane-6-

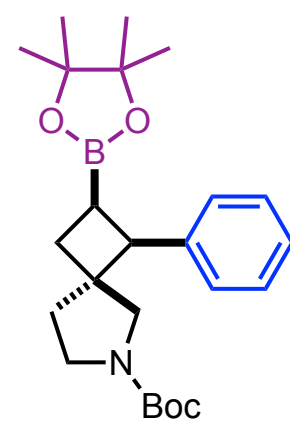
carboxylate (26): The title compound was prepared according to General Procedure A. Purification by silica-gel column chromatography (Gradient: hexane to 20\% EtOAc:hexanes) yields $\mathbf{2 6}$ as colorless oil.

Yield: $45 \mathrm{mg}$ (54\%, major), >20:1 rr, >20:1 $d r$ (99\% NMR yield, 1.7:1 dr) IR (neat): 1691 (s), 1379 (s), 1142 (s), cm ${ }^{-1} ;{ }^{1}$ H NMR (400 MHz, CDCl , mixture of rotamers): $\delta 7.31-7.14(\mathrm{~m}, 5 \mathrm{H}), 3.45-3.26(\mathrm{~m}, 3 \mathrm{H}), 3.16-2.85$ $(\mathrm{m}, 2 \mathrm{H}), 2.54-2.40(\mathrm{~m}, 2 \mathrm{H}), 2.15-2.00(\mathrm{~m}, 3 \mathrm{H}), 1.41(\mathrm{~s}, 9 \mathrm{H}$, minor $), 1.31$ (s, 9H, major), 1.15 (s, 6H, major), 1.14 (s, 6H, minor), 1.00 (s, 6H, major), 0.98 (s, 6H, minor); ${ }^{13} \mathrm{C}$ NMR (126 MHz, $\mathbf{C D C l}_{3}$, mixture of rotamers): $\delta$ 154.7, 154.5, 142.1, 142.0, 128.3, 128.3, 128.3, 128.2, 126.5, 126.3, 83.4, 78.9, 78.8, 53.6, 53.1, 51.6, 51.5, 49.3, 48.5, 44.8, 44.1, 39.4, 38.6, 33.3, 31.8, 28.6, 28.5, 25.1, 24.8; HRMS (ESI+): Calculated for $\mathrm{C}_{24} \mathrm{H}_{36} \mathrm{O}_{4} \mathrm{NBNa}[\mathrm{M}+\mathrm{Na}]+$ : 436.2630, Found: 436.2633. 
tert-Butyl 2-(4-chlorophenyl)-2-phenyl-1-(4,4,5,5-tetramethyl-1,3,2-dioxaborolan-2-yl)-7-

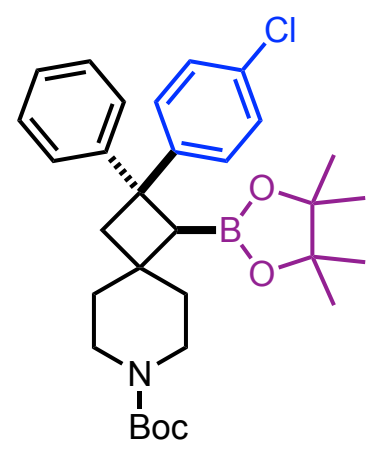
azaspiro[3.5]nonane-7-carboxylate (27): The title compound was prepared according to General Procedure B. Purification by silica-gel column chromatography (Gradient: hexane to 15\% EtOAc:hexanes) yields 27 as colorless oil.

Yield: $63 \mathrm{mg}$ (59\%), >20:1 rr, >20:1 $d r$ (99\% NMR yield, 2.4:1 rr); IR (neat): 1686 (s), 1141 (s), 731 (s) cm $\mathrm{cm}^{-1} ;{ }^{1}$ H NMR (400 MHz, CDCl 3 ): $\delta$ $7.38(\mathrm{~d}, J=7.0 \mathrm{~Hz}, 2 \mathrm{H}), 7.25(\mathrm{t}, J=7.8 \mathrm{~Hz}, 2 \mathrm{H}), 7.17-7.08(\mathrm{~m}, 5 \mathrm{H})$, $3.43-3.33(\mathrm{~m}, 2 \mathrm{H}), 3.22-3.09(\mathrm{~m}, 2 \mathrm{H}), 3.03(\mathrm{~d}, J=11.5 \mathrm{~Hz}, 1 \mathrm{H}), 2.78(\mathrm{~d}, J=2.7 \mathrm{~Hz}, 1 \mathrm{H}), 2.63$ $(\mathrm{dd}, J=11.5,2.8 \mathrm{~Hz}, 1 \mathrm{H}), 1.74-1.69(\mathrm{~m}, 2 \mathrm{H}), 1.42(\mathrm{~s}, 10 \mathrm{H}), 1.34-1.25(\mathrm{~m}, 1 \mathrm{H}), 1.11(\mathrm{~s}, 6 \mathrm{H})$, 0.98 (s, 6H); ${ }^{13} \mathbf{C}$ NMR (101 MHz, $\left.\mathbf{C D C l} 3\right): \delta$ 155.0, 151.6, 148.4, 131.0, 128.6, 128.2, 128.1, 126.3, 125.5, 83.3, 79.3, 47.3, 42.9, 38.4, 36.0, 35.0, 28.6, 25.2, 25.0; HRMS (ESI+): Calculated for $\mathrm{C}_{31} \mathrm{H}_{41} \mathrm{O}_{4} \mathrm{NBClNa}[\mathrm{M}+\mathrm{Na}]+:$ 560.2709, Found: 560.2710.

tert-Butyl 2-(4-(ethoxycarbonyl)phenyl)-2-phenyl-1-(4,4,5,5-tetramethyl-1,3,2-dioxaborolan

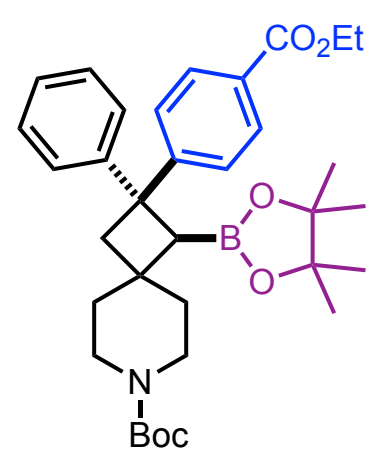

-2-yl)-7-azaspiro[3.5]nonane-7-carboxylate (28): The title compound was prepared according to General Procedure B. Purification by silicagel column chromatography (Gradient: hexane to 15\% EtOAc:hexanes) yields 28 as colorless oil.

Yield: $37 \mathrm{mg}(32 \%),>20: 1 r r,>20: 1 d r(62 \%$ NMR yield, 2.3:1 rr); IR (neat): 1714 (s), 1687 (s), 1271 (s), 1140 (s) cm ${ }^{-1}$; ${ }^{1}$ H NMR (400 MHz, $\left.\mathbf{C D C l}_{3}\right): \delta 7.82(\mathrm{~d}, J=8.4 \mathrm{~Hz}, 2 \mathrm{H}), 7.38(\mathrm{~d}, J=7.0 \mathrm{~Hz}, 2 \mathrm{H}), 7.28-7.20$ (m, 4H), $7.06(\mathrm{t}, J=7.3 \mathrm{~Hz}, 1 \mathrm{H}), 4.31-4.25(\mathrm{~m}, 2 \mathrm{H}), 3.40-3.34(\mathrm{~m}, 2 \mathrm{H}), 3.19-3.09(\mathrm{~m}, 2 \mathrm{H})$, $3.05(\mathrm{~d}, J=11.5 \mathrm{~Hz}, 1 \mathrm{H}), 2.79(\mathrm{~d}, J=2.8 \mathrm{~Hz}, 1 \mathrm{H}), 2.66(\mathrm{dd}, J=11.5,2.8 \mathrm{~Hz}, 1 \mathrm{H}), 1.70-1.66(\mathrm{~m}$, 2H), $1.39(\mathrm{~s}, 10 \mathrm{H}), 1.30(\mathrm{t}, J=7.1 \mathrm{~Hz}, 4 \mathrm{H}), 1.08(\mathrm{~s}, 6 \mathrm{H}), 0.92(\mathrm{~s}, 6 \mathrm{H}) ;{ }^{13} \mathrm{C}$ NMR (101 MHz, $\left.\mathbf{C D C l}_{3}\right): \quad \delta 166.7,155.2,155.1,151.2,129.6,128.6,127.4,126.4,126.4,125.6,83.4,79.3,60.8$, 48.0, 42.8, 38.3, 36.1 , 35.3, 28.6, 25.2, 25.0, 14.4; HRMS (ESI+): Calculated for $\mathrm{C}_{34} \mathrm{H}_{46} \mathrm{O}_{6} \mathrm{NBNa}$ [M+Na]+: 598.3310, Found: 598.3312. 
tert-butyl 2-(benzo[d][1,3]dioxol-5-yl)-2-phenyl-1-(4,4,5,5-tetramethyl-1,3,2-dioxaborolan-2-

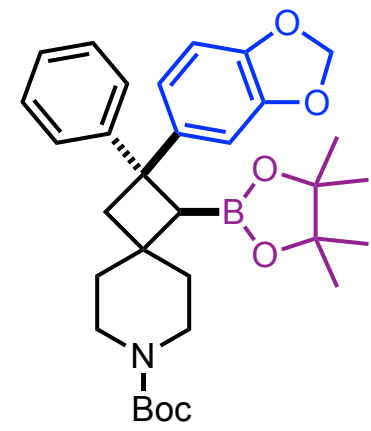
yl)-7-azaspiro[3.5]nonane-7-carboxylate (29): The title compound was prepared according to General Procedure B. Purification by silica-gel column chromatography (Gradient: hexane to 14\% EtOAc:hexanes) yields 29 as colorless oil.

Yield: $70 \mathrm{mg}(64 \%),>12: 1 r r,>20: 1 d r(95 \%$ NMR yield, 2.4:1 rr); IR (neat): 1686 (s), 1141 (s), 731 (w) $\mathrm{cm}^{-1} ;{ }^{1} \mathbf{H}$ NMR (400 MHz, CDCl 3 ): $\delta$ $7.38(\mathrm{~d}, J=6.8 \mathrm{~Hz}, 2 \mathrm{H}), 7.24(\mathrm{t}, J=7.8 \mathrm{~Hz}, 2 \mathrm{H}), 7.08(\mathrm{t}, J=7.3 \mathrm{~Hz}, 1 \mathrm{H})$, $6.72-6.69(\mathrm{~m}, 2 \mathrm{H}), 6.62(\mathrm{~d}, J=8.0 \mathrm{~Hz}, 1 \mathrm{H}), 5.80(\mathrm{dd}, J=5.3,1.4 \mathrm{~Hz}, 2 \mathrm{H}), 3.41-3.35(\mathrm{~m}, 2 \mathrm{H})$, $3.21-3.07(\mathrm{~m}, 2 \mathrm{H}), 2.98(\mathrm{~d}, J=11.5 \mathrm{~Hz}, 1 \mathrm{H}), 2.74(\mathrm{~d}, J=2.7 \mathrm{~Hz}, 1 \mathrm{H}), 2.60(\mathrm{dd}, J=11.5,2.8 \mathrm{~Hz}$, 1H), $1.76-1.67(\mathrm{~m}, 2 \mathrm{H}), 1.41(\mathrm{~s}, 10 \mathrm{H}), 1.31-1.27(\mathrm{~m}, 1 \mathrm{H}), 1.13(\mathrm{~s}, 6 \mathrm{H}), 1.02(\mathrm{~s}, 6 \mathrm{H}) ;{ }^{13} \mathbf{C}$ NMR (101 MHz, CDCl 3 ): $\delta$ 155.1, 152.2, 147.3, 145.1, 144.3, 128.4, 126.2, 125.2, 119.1, 107.8, 100.8, 83.2, 79.2, 47.6, 43.1, 40.9, 38.4, 36.0, 34.8, 28.6, 25.2, 25.1. HRMS (ESI+): Calculated for $\mathrm{C}_{32} \mathrm{H}_{42} \mathrm{O}_{6} \mathrm{NBNa}[\mathrm{M}+\mathrm{Na}]+:$ 570.2997, Found: 570.2999.

tert-Butyl 2,2-diphenyl-1-(4,4,5,5-tetramethyl-1,3,2-dioxaborolan-2-yl)-7-azaspiro[3.5]

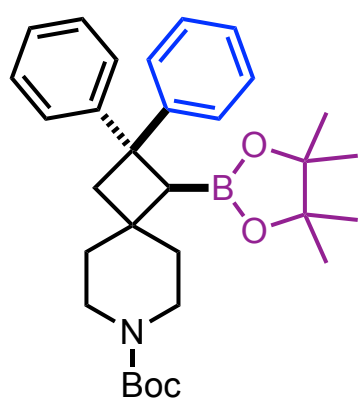

nonane-7-carboxylate (4): The title compound was prepared according to General Procedure B. Purification by silica-gel column chromatography (Gradient: hexane to 15\% EtOAc:hexanes) yields 4 as colorless oil.

Yield: $67 \mathrm{mg}(67 \%),>20: 1 r r,>20: 1 d r(99 \%$ NMR, 2.7:1 rr); IR (neat): 1689 (s), 1243 (w), 1142 (s) cm ${ }^{-1}$; ${ }^{1} \mathbf{H}$ NMR (400 MHz, CDCl$): \delta 7.42$ $(\mathrm{d}, J=7.6 \mathrm{~Hz}, 2 \mathrm{H}), 7.26-7.21(\mathrm{~m}, 4 \mathrm{H}), 7.15(\mathrm{t}, J=7.7 \mathrm{~Hz}, 2 \mathrm{H}), 7.07(\mathrm{t}, J=7.3 \mathrm{~Hz}, 1 \mathrm{H}), 7.00(\mathrm{t}$, $J=7.2 \mathrm{~Hz}, 1 \mathrm{H}), 3.41-3.35(\mathrm{~m}, 2 \mathrm{H}), 3.23-3.12(\mathrm{~m}, 2 \mathrm{H}), 3.08(\mathrm{~d}, J=11.5 \mathrm{~Hz}, 1 \mathrm{H}), 2.80(\mathrm{~d}, J=$ $2.8 \mathrm{~Hz}, 1 \mathrm{H}), 2.64(\mathrm{dd}, J=11.5,2.8 \mathrm{~Hz}, 1 \mathrm{H}), 1.79-1.68(\mathrm{~m}, 2 \mathrm{H}), 1.42(\mathrm{~s}, 9 \mathrm{H}), 1.35-1.25(\mathrm{~m}$, 2H), 1.09 (s, 6H), 0.94 (s, 6H); $\left.{ }^{13} \mathbf{C ~ N M R ~ ( 1 0 1 ~ M H z , ~ C D C l} 3\right): \delta 155.1,152.1,150.0,128.4,128.1$, $126.5,126.4,125.2,125.1,83.1,79.2,47.7,42.8,38.4,36.0,35.0,28.7,25.2,25.0$; HRMS (ESI+): Calculated for $\mathrm{C}_{31} \mathrm{H}_{42} \mathrm{O}_{4} \mathrm{NBNa}[\mathrm{M}+\mathrm{Na}]+:$ 526.3099, Found: 526.3097 . 
tert-Butyl 2-(2-methylprop-1-en-1-yl)-2-phenyl-1-(4,4,5,5-tetramethyl-1,3,2-dioxaborolan-2-

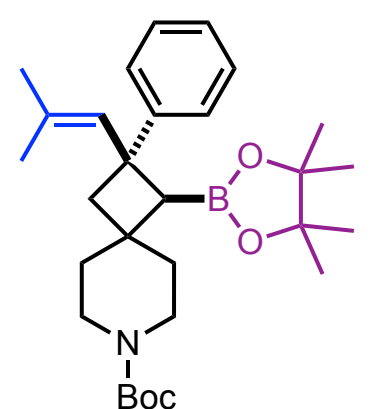
yl)-7-azaspiro[3.5]nonane-7-carboxylate (30): The title compound was prepared according to General Procedure B using 4:1 THF/DMA (0.07M) at $30{ }^{\circ} \mathrm{C}$. Purification by silica-gel column chromatography (Gradient: hexane to 6\% EtOAc:hexanes) yields 30 as colorless oil.

Yield: $55 \mathrm{mg}$ (57\%), 3:1 rr, > 20:1 dr; IR (neat): 1693 (s), 1364 (s), 1142 (s) $\mathrm{cm}^{-1} ;{ }^{1} \mathrm{H}$ NMR (400 $\mathrm{MHz}, \mathrm{CDCl}_{3}$, mixture of 3:1 regioisomers): $\delta$ $7.33-7.31(\mathrm{~m}, 2 \mathrm{H}), 7.25-7.21(\mathrm{~m}, 1 \mathrm{H}), 7.09-7.05(\mathrm{~m}, 2 \mathrm{H}), 5.88(\mathrm{~s}, 1 \mathrm{H}$, major $), 5.56(\mathrm{~d}, J=$ $10.9 \mathrm{~Hz}, 1 \mathrm{H}$, minor), $3.62-3.48(\mathrm{~m}, 2 \mathrm{H}), 3.03-2.91(\mathrm{~m}, 2 \mathrm{H}), 2.63(\mathrm{~d}, J=11.5 \mathrm{~Hz}, 1 \mathrm{H}), 2.43(\mathrm{~d}$, $J=11.4 \mathrm{~Hz}, 1 \mathrm{H}), 2.04(\mathrm{~s}, 1 \mathrm{H}), 1.80-1.78(\mathrm{~m}, 2 \mathrm{H}), 1.58$ (d, $J=1.4 \mathrm{~Hz}, 3 \mathrm{H}), 1.53-1.44$ (m, 2H), 1.42 (s, 9H, minor), 1.41 (s, 9H, major), $1.34(\mathrm{~d}, J=1.3 \mathrm{~Hz}, 3 \mathrm{H}), 1.26(\mathrm{~s}, 12 \mathrm{H}$, major), 1.12 (s, 6H, minor), 1.08 (s, 6H, minor). ${ }^{13} \mathbf{C}$ NMR (126 $\mathbf{M H z}, \mathbf{C D C l}_{3}$, mixture of 3:1 regioisomers): $\delta$ 155.2, 155.1, 151.7, 150.2, 134.2, 132.8, 130.8, 128.12, 128.08, 126.1, 125.6, 124.8, 124.6, 124.5, 83.6, 83.2, 79.22, 79.17, 53.5, 43.69, 43.65, 40.9, 39.9, 38.9, 35.4, 34.9, 29.8, 28.6, 26.8, 26.6, 25.2, 25.2, 24.7, 24.4, 19.0, 18.3. HRMS (ESI+): Calculated for $\mathrm{C}_{29} \mathrm{H}_{44} \mathrm{O}_{4} \mathrm{NBNa}[\mathrm{M}+\mathrm{Na}]+$ : 504.3256, Found: 504.3260.

tert-Butyl 2-phenyl-2-(prop-1-en-2-yl)-1-(4,4,5,5-tetramethyl-1,3,2-dioxaborolan-2-yl)-7-

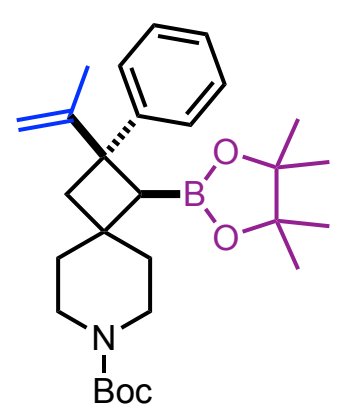
azaspiro[3.5]nonane-7-carboxylate (31): The title compound was prepared according to General Procedure B using 4:1 THF/DMA (0.07M) at $30{ }^{\circ} \mathrm{C}$. Purification by silica-gel column chromatography (Gradient: hexane to $6 \%$ EtOAc:hexanes) yields 31 as colorless oil.

Yield: 39 mg (42\%), 2.3:1 rr, > 20:1 dr; IR (neat): 1696 (s), 1342 (w), 1145 (s) $\mathrm{cm}^{-1}$; ${ }^{1} \mathrm{H}$ NMR (500 MHz, $\mathbf{C D C l}_{3}$, major regioisomer): $\delta 7.35(\mathrm{~d}, J=$ $7.6 \mathrm{~Hz}, 2 \mathrm{H}), 7.30-7.25(\mathrm{~m}, 2 \mathrm{H}), 7.15$ (t, $J=7.2 \mathrm{~Hz}, 1 \mathrm{H}), 4.99$ (s, 1H), 4.74 (s, 1H), $3.49-3.45$ (m, 1H), $3.39-3.36(\mathrm{~m}, 1 \mathrm{H}), 3.15-3.10(\mathrm{~m}, 1 \mathrm{H}), 3.06-3.00(\mathrm{~m}, 1 \mathrm{H}), 2.78(\mathrm{~d}, J=11.5 \mathrm{~Hz}, 1 \mathrm{H})$, $2.45(\mathrm{~s}, 1 \mathrm{H}), 2.35(\mathrm{dd}, J=11.5,2.6 \mathrm{~Hz}, 1 \mathrm{H}), 1.78-1.74(\mathrm{~m}, 1 \mathrm{H}), 1.71-1.66(\mathrm{~m}, 1 \mathrm{H}), 1.43(\mathrm{~s}$, 12H), 1.35 - $1.33(\mathrm{~m}, 2 \mathrm{H}), 1.29(\mathrm{~s}, 6 \mathrm{H}), 1.27$ (s, 6H); ${ }^{13} \mathbf{C}$ NMR (126 MHz, CDCl, major regioisomer): $\delta 155.1,150.9,150.1,128.0,126.8,125.4,109.4,83.0,79.2,49.5,42.0,38.6,36.2$, 34.1, 28.6, 25.3, 25.2, 20.2; HRMS (ESI+): Calculated for $\mathrm{C}_{28} \mathrm{H}_{42} \mathrm{O}_{4} \mathrm{NBNa}[\mathrm{M}+\mathrm{Na}]+$ : 490.3099, Found: 490.3098 . 
tert-Butyl (Z)-2-phenyl-2-(prop-1-en-1-yl)-1-(4,4,5,5-tetramethyl-1,3,2-dioxaborolan-2-yl)-7-

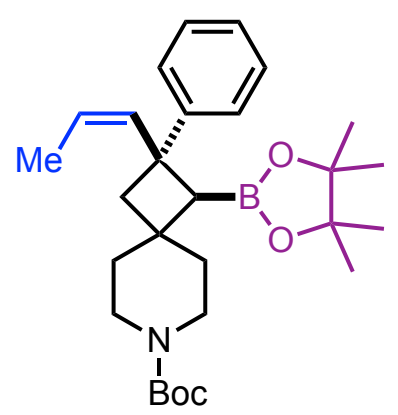
azaspiro[3.5]nonane-7-carboxylate (32): The title compound was prepared according to General Procedure B using 4:1 THF/DMA $(0.07 \mathrm{M})$ at $30{ }^{\circ} \mathrm{C}$. Purification by silica-gel column chromatography (Gradient: hexane to 6\% EtOAc:hexanes) yields 32 as colorless oil.

Yield: $32 \mathrm{mg}$ (34\%), 2.9:1 rr, > 20:1 dr; IR (neat): 1694 (s), 1366 (w), $1144(\mathrm{~s}) \mathrm{cm}^{-1} ;{ }^{1} \mathrm{H}$ NMR (500 $\mathrm{MHz}, \mathrm{CDCl}_{3}$, mixture of 2.9:1 regioisomers): $\delta 7.27(\mathrm{~d}, J=8.1 \mathrm{~Hz}, 2 \mathrm{H}), 7.21-7.18(\mathrm{~m}, 1 \mathrm{H}), 7.06-7.02(\mathrm{~m}, 2 \mathrm{H}), 6.12(\mathrm{~d}, J=$ $11.1 \mathrm{~Hz}, 1 \mathrm{H}$, major $), 5.83-5.78(\mathrm{~m}, 1 \mathrm{H}$, minor $), 5.58-5.51(\mathrm{~m}, 1 \mathrm{H}$, minor $), 5.23-5.17(\mathrm{~m}, 1 \mathrm{H})$, $3.63-3.45(\mathrm{~m}, 2 \mathrm{H}), 2.95-2.83(\mathrm{~m}, 2 \mathrm{H}), 2.58(\mathrm{~d}, J=11.4 \mathrm{~Hz}, 1 \mathrm{H}), 2.46(\mathrm{~d}, J=11.4 \mathrm{~Hz}, 1 \mathrm{H})$, $1.99(\mathrm{~s}, 1 \mathrm{H}), 1.79-1.63(\mathrm{~m}, 2 \mathrm{H}), 1.49-1.40(\mathrm{~m}, 2 \mathrm{H}), 1.37(\mathrm{~s}, 9 \mathrm{H}), 1.31(\mathrm{~d}, J=7.2 \mathrm{~Hz}, 3 \mathrm{H}), 1.21$ (s, 12H, major), 1.06 (s, 6H, minor), 1.01 (s, 6H, minor); ${ }^{13} \mathbf{C ~ N M R ~ ( 1 2 6 ~ M H z , ~ C D C l ~} 3$, mixture of 2.9:1 regioisomers): $\delta 155.2,155.1,151.3,149.8,139.6,130.0,128.2,128.1,125.9,125.6$, $125.1,124.8,123.7,83.6,83.2,79.3,79.2,52.2$, 44.0, 43.5, 40.0, 39.9, 39.8, 38.9, 35.2, 29.8, 28.6, 25.2, 25.2, 25.1, 24.6, 24.5, 14.3, 13.4; HRMS (ESI+): Calculated for $\mathrm{C}_{28} \mathrm{H}_{42} \mathrm{O}_{4} \mathrm{NBNa}[\mathrm{M}+\mathrm{Na}]+$ : 490.3099, Found: 490.3103 .

tert-Butyl 2-phenyl-1-(4,4,5,5-tetramethyl-1,3,2-dioxaborolan-2-yl)-2-(4-(trifluoromethyl)

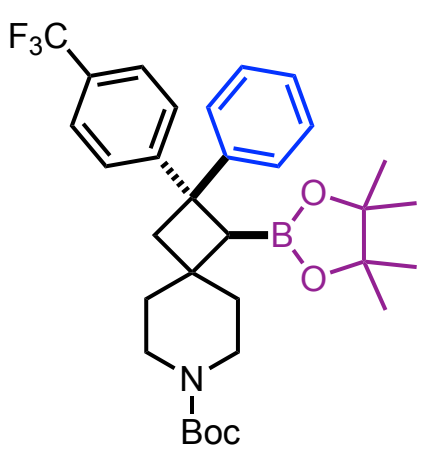
phenyl)-7-azaspiro[3.5]nonane-7-carboxylate (33): The title compound was prepared according to General Procedure B. Purification by silica-gel column chromatography (Gradient: hexane to $15 \%$ EtOAc:hexanes) yields $\mathbf{3 3}$ as colorless oil.

Yield: $48 \mathrm{mg}$ (42\%), >20:1 rr, >20:1 $d r$ (72\% NMR yield, 2.9:1 rr); IR (neat): 1688 (s), 1324 (s), 1121 (s) cm $\mathrm{cm}^{-1}{ }^{1}$ H NMR (400 MHz, $\left.\mathbf{C D C l}_{3}\right): \delta 7.54-7.49(\mathrm{~m}, 4 \mathrm{H}), 7.23-7.16(\mathrm{~m}, 4 \mathrm{H}), 7.06-7.02(\mathrm{~m}$, $1 \mathrm{H}), 3.43-3.37(\mathrm{~m}, 2 \mathrm{H}), 3.21-3.11(\mathrm{~m}, 3 \mathrm{H}), 2.77(\mathrm{~d}, J=2.7 \mathrm{~Hz}, 1 \mathrm{H}), 2.63(\mathrm{dd}, J=11.6,2.7 \mathrm{~Hz}$, $1 \mathrm{H}), 1.78-1.71(\mathrm{~m}, 2 \mathrm{H}), 1.42(\mathrm{~s}, 9 \mathrm{H}), 1.38-1.27(\mathrm{~m}, 2 \mathrm{H}), 1.10(\mathrm{~s}, 6 \mathrm{H}), 0.95(\mathrm{~s}, 6 \mathrm{H}) ;{ }^{13} \mathbf{C}$ NMR (126 MHz, CDCI3): $\delta$ 156.3, 155.1, 148.8, 128.4, 127.6 (q, $J=32.5$ Hz), 126.8, 126.5, 125.6, 125.5 (q, $J=3.5 \mathrm{~Hz}), 125.5$ (q, $J=270.0 \mathrm{~Hz}), 83.3,79.4,47.9,43.0,38.5,35.9,35.2,28.6,25.2$, 25.0; ${ }^{19}$ F NMR (376 MHz, $\mathbf{C D C l}_{3}$ ): $\delta$-62.29; HRMS (ESI+): Calculated for $\mathrm{C}_{32} \mathrm{H}_{41} \mathrm{O}_{4} \mathrm{NBF}_{3} \mathrm{Na}$ [M+Na]+: 594.2973, Found: 594.2973. 
tert-Butyl 2-(2-methylprop-1-en-1-yl)-1-(4,4,5,5-tetramethyl-1,3,2-dioxaborolan-2-yl)-2-(4-

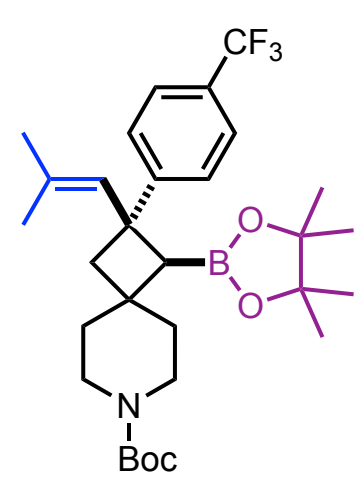
(trifluoromethyl)phenyl)-7-azaspiro[3.5]nonane-7-carboxylate (34): The title compound was prepared according to General Procedure B using 4:1 THF/DMA $(0.07 \mathrm{M})$ at $30{ }^{\circ} \mathrm{C}$. Purification by silica-gel column chromatography (Gradient: hexane to 6\% EtOAc:hexanes) yields 34 as colorless oil.

Yield: $60 \mathrm{mg}$ (53\%), 2.9:1 rr, > 20:1 dr; IR (neat): 1693 (s), 1324 (s), 1142 (s), $1122(\mathrm{~s}) \mathrm{cm}^{-1} ; \mathbf{1 H}$ NMR (400 $\mathrm{MHz}, \mathrm{CDCl}_{3}$, mixture of 2.9:1 regioisomers): $\delta 7.51(\mathrm{~d}, J=8.2 \mathrm{~Hz}, 2 \mathrm{H}), 7.44(\mathrm{~d}, J=8.4 \mathrm{~Hz}, 2 \mathrm{H}), 7.18(\mathrm{~d}, J=8.0 \mathrm{~Hz}, 2 \mathrm{H}$, minor), 5.93 (s, 1H, major), 5.57 (d, $J=10.9 \mathrm{~Hz}, 1 \mathrm{H}$, minor), $3.69-3.57(\mathrm{~m}, 2 \mathrm{H}), 3.02-2.90(\mathrm{~m}, 2 \mathrm{H})$, $2.64(\mathrm{~d}, J=11.0 \mathrm{~Hz}, 1 \mathrm{H}), 2.46(\mathrm{~d}, J=11.4 \mathrm{~Hz}, 1 \mathrm{H}), 1.98(\mathrm{~s}, 1 \mathrm{H}), 1.83-1.79(\mathrm{~m}, 2 \mathrm{H}), 1.61$ (s, $3 \mathrm{H}), 1.48(\mathrm{~s}, 2 \mathrm{H}), 1.44(\mathrm{~s}, 9 \mathrm{H}), 1.34(\mathrm{~s}, 3 \mathrm{H}), 1.28$ (s, 12H, major), 1.15 (s, 6H, minor), 1.11 (s, 6H, minor). ${ }^{13} \mathrm{C}$ NMR (126 $\mathrm{MHz} \mathrm{CDCl}_{3}$, mixture of 2.9:1 regioisomers): $\delta$ 155.7, 155.2, 155.0, 154.6, 133.4, 132.1, 127.2, 126.9, 126.3, 125.9, 125.7, 125.1 (q, $J=3.8 \mathrm{~Hz}$, major), 125.0 (q, $J=$ 3.7 Hz, minor), 124.0, 123.5, 83.9, 83.3, 79.32, 79.28, 54.0, 43.7, 43.6, 40.8, 40.1, 40.0, 38.8, 35.1, 32.1, 29.8, 28.6, 26.7, 26.5, 25.2, 25.17, 24.8, 24.5, 19.1, 18.3; ${ }^{19}$ F NMR (471 MHz, CDCl 3 ): $\delta$ 62.07 (minor), -62.13 (major); HRMS (ESI+): Calculated for $\mathrm{C}_{30} \mathrm{H}_{43} \mathrm{O}_{4} \mathrm{NBF}_{3} \mathrm{Na}[\mathrm{M}+\mathrm{Na}]+$ : 572.3129, Found: 572.3133.

tert-butyl 2-(benzo[d][1,3]dioxol-5-yl)-2-phenyl-1-(4,4,5,5-tetramethyl-1,3,2-dioxaborolan-2-

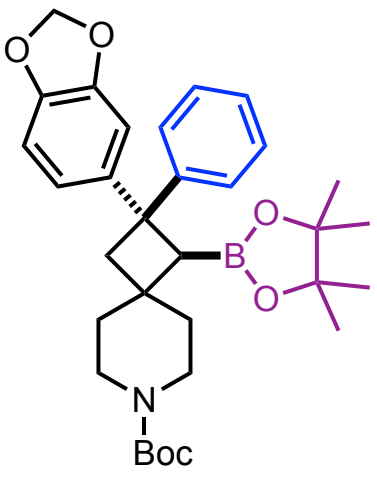
yl)-7-azaspiro[3.5]nonane-7-carboxylate (35): The title compound was prepared according to General Procedure B. Purification by silicagel column chromatography (Gradient: hexane to 14\% EtOAc:hexanes) yields 35 as colorless oil.

Yield: $66 \mathrm{mg}(60 \%),>20: 1 r r,>20: 1 d r(89 \%$ NMR yield, 2.3:1 rr); IR (neat): 1686 (s), 1141 (s), 700 (w) cm ${ }^{-1} ;{ }^{1}$ H NMR (400 MHz, CDCl 3 ): $\delta 7.04-7.00(\mathrm{~m}, 1 \mathrm{H}), 6.91(\mathrm{dd}, J=8.2,1.9 \mathrm{~Hz}, 1 \mathrm{H}), 6.86(\mathrm{~d}, J=1.9 \mathrm{~Hz}$, $1 \mathrm{H}), 6.69(\mathrm{~d}, J=8.1 \mathrm{~Hz}, 1 \mathrm{H}), 5.86(\mathrm{dd}, J=8.1,1.5 \mathrm{~Hz}, 2 \mathrm{H}), 3.41-3.35(\mathrm{~m}, 2 \mathrm{H}), 3.19-3.12(\mathrm{~m}$, 2H), 3.02 (d, $J=11.5 \mathrm{~Hz}, 1 \mathrm{H}), 2.69$ (d, $J=2.8 \mathrm{~Hz}, 1 \mathrm{H}), 2.56(\mathrm{dd}, J=11.5,2.9 \mathrm{~Hz}, 1 \mathrm{H}), 1.73-$ 1.69 (m, 2H), 1.42 (s, 11H), 1.08 (s, 6H), 0.93 (s, 6H); ${ }^{13} \mathbf{C}$ NMR (101 MHz, CDCl $): \delta$ 155.1, $147.7,146.4,145.1,128.1,126.4,125.2$, 119.2, 108.0, 107.4, 100.9, 83.2, 79.2, 47.6, 42.8, 38.4, 
36.0, 34.9, 28.6, 25.2, 25.0. HRMS (ESI+): Calculated for $\mathrm{C}_{32} \mathrm{H}_{42} \mathrm{O}_{6} \mathrm{NBNa}[\mathrm{M}+\mathrm{Na}]+$ : 570.2997, Found: 570.2994 .

tert-Butyl 2-(6-methoxypyridin-2-yl)-2-phenyl-1-(4,4,5,5-tetramethyl-1,3,2-dioxaborolan-2-

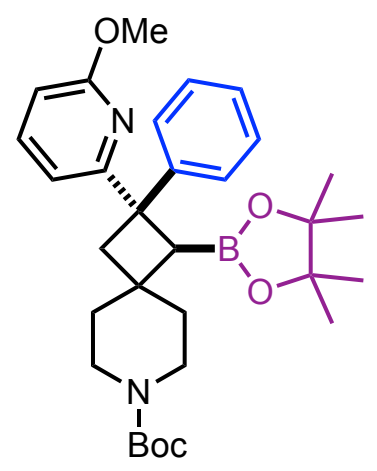
yl)-7-azaspiro[3.5]nonane-7-carboxylate (36): The title compound was prepared according to General Procedure B. Purification by silica-gel column chromatography (Gradient: hexane to 15\% EtOAc:hexanes) yields 36 as colorless oil.

Yield: $22 \mathrm{mg}(21 \%),>20: 1 r r,>20: 1 d r(42 \%$ NMR yield, 1.5:1 rr); IR (neat): 1692 (s), 1364 (s), 1144 (s) cm ${ }^{-1} ;{ }^{1} \mathbf{H}$ NMR (400 MHz, CDCl 3 ): $\delta$ $7.50(\mathrm{~d}, J=1.4 \mathrm{~Hz}, 2 \mathrm{H}), 7.32-7.28(\mathrm{~m}, 3 \mathrm{H}), 7.14(\mathrm{t}, J=7.3 \mathrm{~Hz}, 1 \mathrm{H})$, 6.49 (s, 1H), 6.39 (d, $J=8.1 \mathrm{~Hz}, 1 \mathrm{H}), 3.93(\mathrm{~s}, 3 \mathrm{H}), 3.55-3.50(\mathrm{~m}, 1 \mathrm{H}), 3.41(\mathrm{~s}, 1 \mathrm{H}), 3.17-3.07$ (m, 3H), $2.66-2.63(\mathrm{~m}, 2 \mathrm{H}), 1.94-1.86(\mathrm{~m}, 1 \mathrm{H}), 1.80-1.74(\mathrm{~m}, 1 \mathrm{H}), 1.43(\mathrm{~s}, 10 \mathrm{H}), 1.35-1.34$ (m, 1H), 1.07 (s, 6H), 0.99 (s, 6H); ${ }^{13} \mathbf{C}$ NMR (126 MHz, CDCl $): \delta 165.9,163.1,155.1,150.5$, $138.6,128.1,127.2$, 125.5, 113.6, 107.1, 82.8, 79.2, 53.5, 49.2, 42.2, 39.4, 35.6, 34.6, 28.6, 25.1, 25.1; HRMS (ESI+): Calculated for $\mathrm{C}_{31} \mathrm{H}_{44} \mathrm{O}_{5} \mathrm{~N}_{2} \mathrm{~B}$ [M+H]+: 535.3338, Found: 535.3345.

tert-Butyl 2-ethyl-2-phenyl-1-(4,4,5,5-tetramethyl-1,3,2-dioxaborolan-2-yl)-7-azaspiro

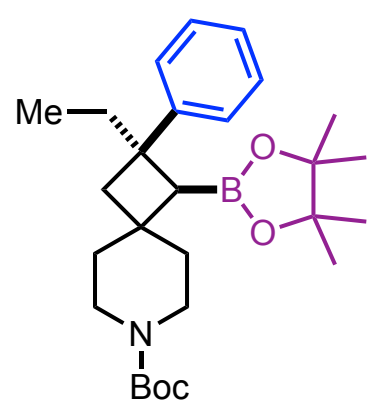

[3.5]nonane-7-carboxylate (37): The title compound was prepared according to General Procedure B. Purification by silica-gel column chromatography (Gradient: hexane to 14\% EtOAc:hexanes) yields 37 as colorless oil.

Yield: 49 mg (54\%), >20:1 rr, >20:1 $d r$ (99\% NMR yield, 1.1:1 rr); IR (neat): 1690 (s), 1242 (s), 1142 (s) cm ${ }^{-1} ;{ }^{1}$ H NMR (400 MHz, CDCl 3 ): $\delta 7.23(\mathrm{t}, J=7.6 \mathrm{~Hz}, 2 \mathrm{H}), 7.15(\mathrm{~d}, J=6.8 \mathrm{~Hz}, 2 \mathrm{H}), 7.10(\mathrm{t}, J=7.2 \mathrm{~Hz}, 1 \mathrm{H}), 3.55-3.52(\mathrm{~m}, 1 \mathrm{H})$, $3.44-3.41(\mathrm{~m}, 1 \mathrm{H}), 3.23-3.17(\mathrm{~m}, 1 \mathrm{H}), 3.12-3.06(\mathrm{~m}, 1 \mathrm{H}), 2.48(\mathrm{~d}, J=12.1 \mathrm{~Hz}, 1 \mathrm{H}), 1.99-$ $1.89(\mathrm{~m}, 4 \mathrm{H}), 1.73-1.68(\mathrm{~m}, 2 \mathrm{H}), 1.64-1.60(\mathrm{~m}, 2 \mathrm{H}), 1.44(\mathrm{~s}, 9 \mathrm{H}), 1.09(\mathrm{~s}, 6 \mathrm{H}), 0.93(\mathrm{~s}, 6 \mathrm{H})$, 0.47 (t, $J=7.2 \mathrm{~Hz}, 3 \mathrm{H}) ;{ }^{13} \mathbf{C}$ NMR (101 MHz, $\left.\mathbf{C D C l}_{3}\right): \delta 155.2,148.0,127.8,126.8,125.1,82.9$, 79.3, 42.7, 40.7, 40.1, 38.3, 36.9, 33.1, 28.6, 25.4, 25.0, 8.5; HRMS (ESI+): Calculated for $\mathrm{C}_{27} \mathrm{H}_{42} \mathrm{O}_{4} \mathrm{NBNa}[\mathrm{M}+\mathrm{Na}]+:$ 478.3099, Found: 478.3102. 


\section{Further Transformations:}

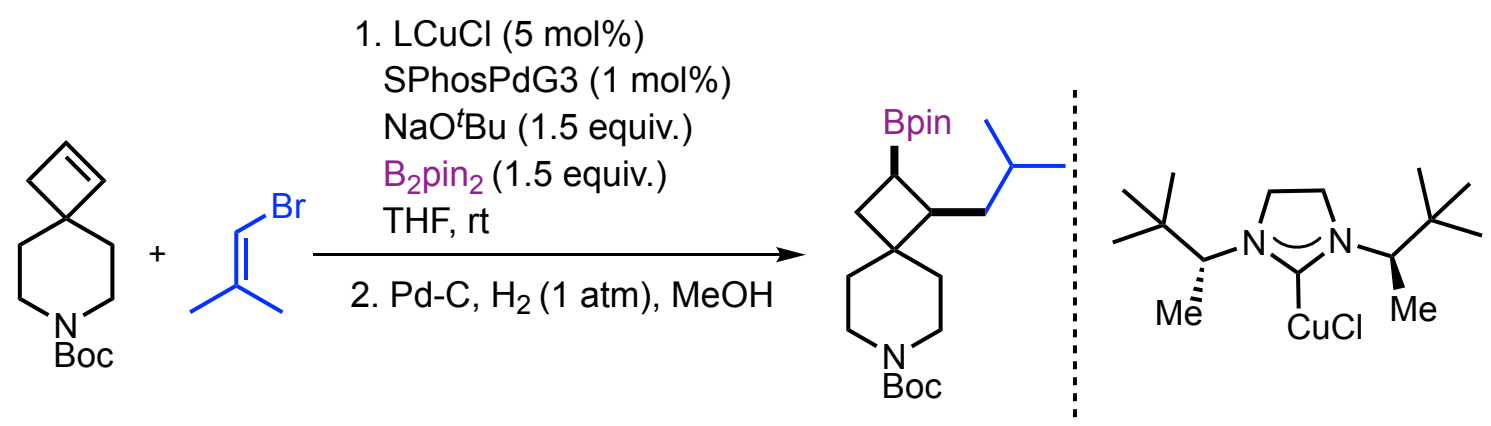

tert-Butyl 1-isobutyl-2-(4,4,5,5-tetramethyl-1,3,2-dioxaborolan-2-yl)-7-azaspiro[3.5]nonane-

7-carboxylate (38): The alkenylboration was performed according to General Procedure A. Purification by silica-gel column chromatography (Gradient: hexane to 14\% EtOAc:hexanes) afforded the alkenylboration product as colorless oil which was used in the next step without any characterization. In an oven dried 10-mL round-bottom flask, the alkene was taken in $\mathrm{MeOH}(2.0$ $\mathrm{mL}$ ) under positive pressure of $\mathrm{N}_{2}$. Pd-C (5 mol\%) was added and hydrogen gas was bubbled through the solution for $5 \mathrm{~min}$. The heterogeneous mixture was stirred at room temperature until TLC shows complete consumption of starting alkene. The reaction contents were filtered over celite and washed with EtOAc to obtain $\mathbf{3 8}$ as colorless oil.

Yield: 46 mg (57\%), >20:1 rr, >20:1 dr; IR (neat): 1693 (s), 1242 (w), 1143 (s) cm ${ }^{-1}$; ${ }^{1}$ H NMR (400 MHz, $\left.\mathbf{C D C l}_{3}\right): \delta 3.81(\mathrm{~s}, 2 \mathrm{H}), 2.84-2.77(\mathrm{~m}, 2 \mathrm{H}), 2.32-2.25(\mathrm{~m}, 1 \mathrm{H}), 1.90-1.72(\mathrm{~m}, 4 \mathrm{H})$, $1.49-1.45(\mathrm{~m}, 3 \mathrm{H}), 1.43(\mathrm{~s}, 9 \mathrm{H}), 1.40-1.30(\mathrm{~m}, 2 \mathrm{H}), 1.23(\mathrm{~d}, J=2.5 \mathrm{~Hz}, 12 \mathrm{H}), 1.19-1.14(\mathrm{~m}$, $1 \mathrm{H}), 0.84(\mathrm{~d}, J=6.6 \mathrm{~Hz}, 3 \mathrm{H}), 0.81(\mathrm{~d}, J=6.5 \mathrm{~Hz}, 3 \mathrm{H}) ;{ }^{13} \mathbf{C}$ NMR (126 MHz, CDCl $): \delta 155.1$, 83.1, 79.1, 43.3, 39.6, 39.2, 38.4, 31.1, 29.5, 28.6, 26.1, 25.1, 23.1, 22.7; HRMS (ESI+): Calculated for $\mathrm{C}_{23} \mathrm{H}_{42} \mathrm{O}_{4} \mathrm{NBNa}[\mathrm{M}+\mathrm{Na}]+:$ 430.3099, Found: 430.3106 .

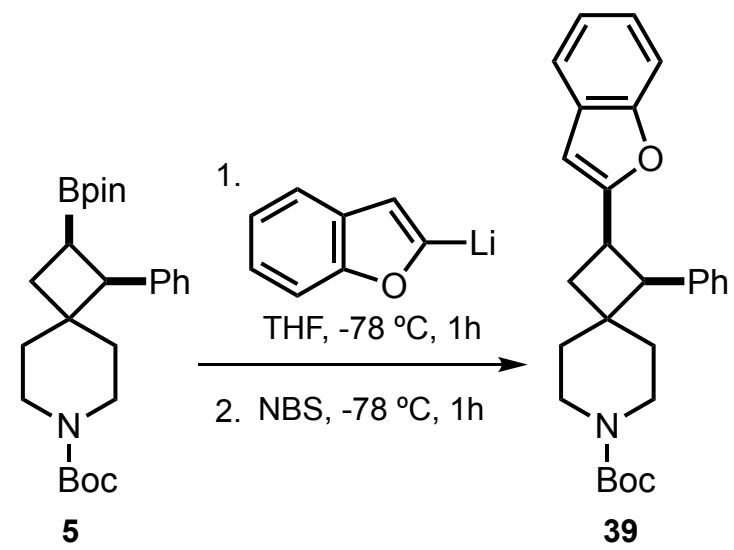


tert-Butyl 2-(benzofuran-2-yl)-1-phenyl-7-azaspiro[3.5]nonane-7-carboxylate (39): To an oven-dried 13 x 100 mm screw-cap vial was added benzofuran (24.0 $\mu \mathrm{L}, 0.22$ mmol, 2.2 equiv.). The vial was then inerted with three evacuation/backfill $\left(\mathrm{N}_{2}\right)$ cycles and sealed with a septum. THF $(0.5 \mathrm{~mL})$ was then added via syringe and the solution was cooled to $-78{ }^{\circ} \mathrm{C}$ before the addition of $2.0 \mathrm{M} n$-BuLi in hexane $(0.10 \mathrm{~mL}, 0.20 \mathrm{mmol}, 2.0$ equiv. $)$ dropwise over $5 \mathrm{~min}$. The resulting mixture was warmed to $\mathrm{rt}$ and stirred for $1 \mathrm{~h}$. Then a solution of 5 (43.0 mg, $0.10 \mathrm{mmol}, 1.0$ equiv.) was added dropwise to the solution of lithiated benzofuran at $-78^{\circ} \mathrm{C}$. After $1 \mathrm{~h}$ at $-78{ }^{\circ} \mathrm{C}$, a solution of NBS (36.0 mg, $0.20 \mathrm{mmol}, 2.0$ equiv.) in THF (0.5 mL) was added dropwise over $5 \mathrm{~min}$. After stirring at $-78{ }^{\circ} \mathrm{C}$ for $1 \mathrm{~h}$, a $20 \%$ aqueous solution of $\mathrm{Na}_{2} \mathrm{~S}_{2} \mathrm{O}_{3}(1.00 \mathrm{~mL})$ was added. The mixture was warmed to room temperature and extracted with ethyl acetate $(3 \times 10 \mathrm{~mL})$. The combined organic layers were dried over $\mathrm{Na}_{2} \mathrm{SO}_{4}$ and concentrated in vacuo. The crude material was purified by flash column chromatography (Gradient: hexane to 5\% EtOAc:hexanes) to obtain 39 as a white solid (13.0 mg 31\% yield).

IR (neat): 1692 (s), 1173 (s), 1152 (s) cm ${ }^{-1} ;{ }^{1} \mathbf{H}$ NMR (500 MHz, CDCl $): \delta 7.46-7.44$ (m, 1H), $7.28-7.26(\mathrm{~m}, 1 \mathrm{H}), 7.16-7.13(\mathrm{~m}, 2 \mathrm{H}), 7.08-7.04(\mathrm{~m}, 5 \mathrm{H}), 6.41(\mathrm{~s}, 1 \mathrm{H}), 4.22(\mathrm{dd}, J=9.6,1.4$ $\mathrm{Hz}, 1 \mathrm{H}), 3.66(\mathrm{dd}, J=9.7,1.9 \mathrm{~Hz}, 2 \mathrm{H}), 3.36-3.31(\mathrm{~m}, 1 \mathrm{H}), 3.19-3.16(\mathrm{~m}, 1 \mathrm{H}), 3.06-3.00(\mathrm{~m}$, $1 \mathrm{H}), 2.51(\mathrm{dd}, J=11.8,9.3 \mathrm{~Hz}, 1 \mathrm{H}), 2.44-2.39(\mathrm{~m}, 1 \mathrm{H}), 1.88(\mathrm{t}, J=5.7 \mathrm{~Hz}, 2 \mathrm{H}), 1.53-1.48(\mathrm{~m}$, 1H), 1.42 (s, 9H), 1.27 - $1.21(\mathrm{~m}, 1 \mathrm{H}) ;{ }^{13} \mathbf{C}$ NMR (126 MHz, CDCl $): \delta 159.1,155.0,154.9$, 138.0, 129.6, 128.7, 127.8, 126.5, 123.3, 122.5, 120.4, 110.9, 103.8, 79.4, 53.9, 40.0, 38.8, 34.0, 33.6, 32.7, 28.6; HRMS (ESI+): Calculated for $\mathrm{C}_{27} \mathrm{H}_{31} \mathrm{O}_{3} \mathrm{NNa}[\mathrm{M}+\mathrm{Na}]+$ : 440.2196, Found: 440.2196 .

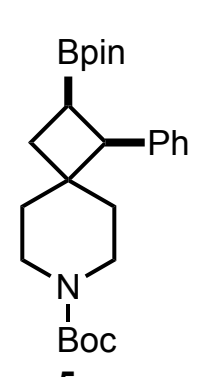

5

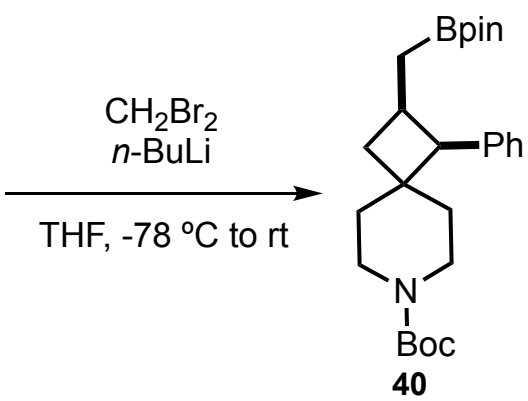

40

tert-Butyl 1-phenyl-2-((4,4,5,5-tetramethyl-1,3,2-dioxaborolan-2-yl)methyl)-7-azaspiro

[3.5]nonane-7-carboxylate (40): To an oven-dried 13 x $100 \mathrm{~mm}$ screw-cap vial was added boronic ester 5 (95.0 $\mathrm{mg}, 0.22 \mathrm{mmol}, 1.0$ equiv.). The vial was then inerted with three evacuation/backfill $\left(\mathrm{N}_{2}\right)$ cycles and sealed with a septum. THF $(2.2 \mathrm{~mL})$, and $\mathrm{CH}_{2} \mathrm{Br}_{2}(39.0 \mu \mathrm{L}$, 
$0.56 \mathrm{mmol}, 2.5$ equiv.) were then added via syringe. The solution was cooled to $-78{ }^{\circ} \mathrm{C}$ before the addition of $2.0 \mathrm{M} \mathrm{n}$-BuLi in hexane ( $0.24 \mathrm{~mL}, 0.48 \mathrm{mmol}, 2.2$ equiv.) dropwise over $5 \mathrm{~min}$. The reaction was stirred for $5 \mathrm{~min}$ at $-78^{\circ} \mathrm{C}$ before warming to $25^{\circ} \mathrm{C}$. The reaction was allowed to stir for $18 \mathrm{~h}$ at $25^{\circ} \mathrm{C}$ before being quenched upon the addition of $\mathrm{H}_{2} \mathrm{O}(3.0 \mathrm{~mL})$. The aqueous layer was then extracted with EtOAc $(3 \times 5 \mathrm{~mL})$, and the combined organic layers were dried over $\mathrm{Na}_{2} \mathrm{SO}_{4}$, filtered, and concentrated under reduced pressure. Purification via silica gel chromatography (Gradient: hexane to 14\% EtOAc:hexanes) yield 40 as a colorless oil (96.0 mg, $99 \%$ yield).

IR (neat): 1699 (s), 1145 (s), cm ${ }^{-1} ;{ }^{1} \mathbf{H}$ NMR (400 MHz, CDCl 3$): \delta 7.29-7.21$ (m, 4H), 7.19 $7.15(\mathrm{~m}, 1 \mathrm{H}), 3.52-3.46(\mathrm{~m}, 1 \mathrm{H}), 3.42-3.36(\mathrm{~m}, 1 \mathrm{H}), 3.28(\mathrm{dd}, J=9.3,2.6 \mathrm{~Hz}, 1 \mathrm{H}), 3.07-2.91$ $(\mathrm{m}, 3 \mathrm{H}), 2.16-2.10(\mathrm{~m}, 1 \mathrm{H}), 1.82-1.78(\mathrm{~m}, 2 \mathrm{H}), 1.72(\mathrm{t}, J=10.7 \mathrm{~Hz}, 1 \mathrm{H}), 1.40(\mathrm{~s}, 10 \mathrm{H}), 1.31-$ $1.24(\mathrm{~m}, 1 \mathrm{H}), 1.10(\mathrm{~s}, 6 \mathrm{H}), 1.05(\mathrm{~s}, 6 \mathrm{H}), 1.01(\mathrm{dd}, J=9.2,7.5 \mathrm{~Hz}, 1 \mathrm{H}), 0.85(\mathrm{dd}, J=16.1,8.6 \mathrm{~Hz}$, 1H); ${ }^{13} \mathbf{C}$ NMR (101 MHz, $\left.\mathbf{C D C l}_{3}\right): \delta$ 155.0, 139.3, 131.0, 127.7, 126.0, 82.8, 79.1, 54.3, 40.8, 39.9, 38.5, 33.5, 29.6, 28.5, 24.9, 24.6. HRMS (ESI+): Calculated for $\mathrm{C}_{26} \mathrm{H}_{40} \mathrm{O}_{4} \mathrm{NBNa}[\mathrm{M}+\mathrm{Na}]+$ : 464.2943, Found: 464.2946.

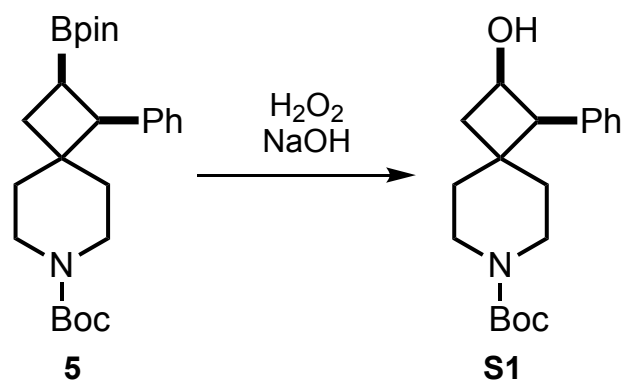

tert-Butyl 2-hydroxy-1-phenyl-7-azaspiro[3.5]nonane-7-carboxylate (S1): Bpin 5 (78.0 mg, $0.18 \mathrm{mmol}, 1.0$ equiv.) was taken in $2 \mathrm{~mL} \mathrm{THF}$, cooled to $0{ }^{\circ} \mathrm{C}$ (ice bath) followed by the addition of $1.0 \mathrm{~mL} 2 \mathrm{M} \mathrm{NaOH}$ (aq.) and $0.5 \mathrm{~mL} \mathrm{H}_{2} \mathrm{O}_{2}$. After $1 \mathrm{~h}, 10 \mathrm{~mL}$ water was added, and the aqueous layer was extracted with EtOAc $(3 \times 10 \mathrm{~mL})$. Combined organic layer was washed with brine (10 $\mathrm{mL}$ ), dried over $\mathrm{Na}_{2} \mathrm{SO}_{4}$ and concentrated in reduced pressure to obtain $\mathbf{S 1}$ as a clear oil (55.0 mg, $0.173 \mathrm{mmol}, 96 \%$ avg. isolated yield).

IR (neat): 3411 (w), 1666 (s), 1425 (s), 1243 (s) cm ${ }^{-1} ;{ }^{1}$ H NMR (400 MHz, CDCl $)$ ): $\delta 7.31$ $7.20(\mathrm{~m}, 4 \mathrm{H}), 7.21-7.12(\mathrm{~m}, 1 \mathrm{H}), 4.66-4.56(\mathrm{~m}, 1 \mathrm{H}), 3.52-3.43(\mathrm{~m}, 1 \mathrm{H}), 3.31(\mathrm{~d}, J=7.2 \mathrm{~Hz}$, $1 \mathrm{H}), 3.19-3.03(\mathrm{~m}, 2 \mathrm{H}), 2.96-2.86(\mathrm{~m}, 1 \mathrm{H}), 2.30-2.21(\mathrm{~m}, 1 \mathrm{H}), 2.10(\mathrm{~s}, 1 \mathrm{H}), 1.98-1.90(\mathrm{~m}$, 1H), $1.65-1.54(\mathrm{~m}, 2 \mathrm{H}), 1.42-1.38(\mathrm{~m}, 1 \mathrm{H}), 1.32(\mathrm{~s}, 9 \mathrm{H}), 1.24-1.18(\mathrm{~m}, 1 \mathrm{H}) ;{ }^{13} \mathbf{C}$ NMR (101 
MHz, $\left.\mathbf{C D C l}_{3}\right): \delta 155.0,136.6,130.7,128.2,126.7,79.4,65.4,56.4,41.2,39.1,37.3,33.4,28.5$; HRMS (ESI+): Calculated for $\mathrm{C}_{19} \mathrm{H}_{27} \mathrm{O}_{3} \mathrm{NNa}[\mathrm{M}+\mathrm{Na}]+:$ 340.1883, Found: 340.1884 .

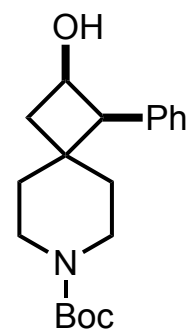

S1

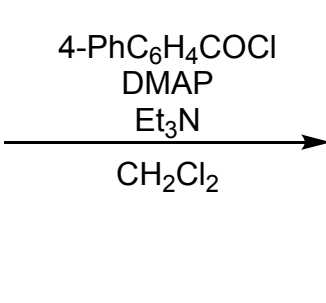

$\mathrm{Et}_{3} \mathrm{~N}$

$\mathrm{CH}_{2} \mathrm{Cl}_{2}$

tert-Butyl

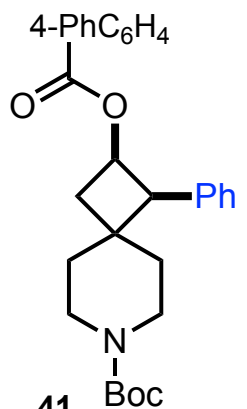

41 Boc

carboxylate (41): In an oven dried 2 dram vial, alcohol S1 (20.0 mg, $0.063 \mathrm{mmol}, 1.0$ equiv.), [1,1'-biphenyl]-4-carbonyl chloride (23.0 mg, 0.107 mmol, 1.7 equiv.) and DMAP (1.5 mg, 0.0126 mmol, 0.20 equiv.) were taken under positive pressure of $\mathrm{N}_{2}$. Dichloromethane $(1.0 \mathrm{~mL})$ and triethylamine $(26.0 \mu \mathrm{L}, 0.189 \mathrm{mmol}, 3.0$ equiv.) were added and the reaction mixture was stirred at room temperature for $24 \mathrm{~h}$. Solvent was removed and purified using silica-gel column chromatography to obtain the ester 41 (20.0 mg, 64\% yield). This compound was crystallized from EtOAc/Pentane at room temperature.

IR (neat): 1714 (w), 1686 (s), 1265 (s), 1096 (s) cm ${ }^{-1} ;{ }^{1}$ H NMR (400 MHz, CDCl 3 ): $\delta 7.89$ (d, $J$ $=8.2 \mathrm{~Hz}, 2 \mathrm{H}), 7.53-7.51(\mathrm{~m}, 4 \mathrm{H}), 7.38(\mathrm{t}, J=7.6 \mathrm{~Hz}, 2 \mathrm{H}), 7.31(\mathrm{~d}, J=7.5 \mathrm{~Hz}, 3 \mathrm{H}), 7.20(\mathrm{t}, J=$ $8.4 \mathrm{~Hz}, 2 \mathrm{H}), 7.12$ (t, $J=7.3 \mathrm{~Hz}, 1 \mathrm{H}), 5.62$ (q, $J=7.7 \mathrm{~Hz}, 1 \mathrm{H}), 3.68$ (dd, $J=8.0,2.7 \mathrm{~Hz}, 1 \mathrm{H}), 3.56$ - $3.49(\mathrm{~m}, 1 \mathrm{H}), 3.28$ - $3.22(\mathrm{~m}, 1 \mathrm{H}), 3.15-3.09(\mathrm{~m}, 1 \mathrm{H}), 2.98-2.91(\mathrm{~m}, 1 \mathrm{H}), 2.53-2.47(\mathrm{~m}$, 1H), $2.32-2.27(\mathrm{~m}, 1 \mathrm{H}), 1.81-1.73(\mathrm{~m}, 2 \mathrm{H}), 1.35(\mathrm{~s}, 11 \mathrm{H}) ;{ }^{13} \mathbf{C}$ NMR (101 MHz, CDCl$): \delta$ $165.7,155.0,145.8,140.1,136.1,130.5,130.2,129.1,128.9,128.3,128.2,127.4,127.1,126.8$, 79.5, 67.8, 55.4, 39.1, 38.8, 38.2, 28.6; HRMS (ESI+): Calculated for $\mathrm{C}_{32} \mathrm{H}_{35} \mathrm{O}_{4} \mathrm{NNa}[\mathrm{M}+\mathrm{Na}]+$ : 520.2458, Found: 520.2457.

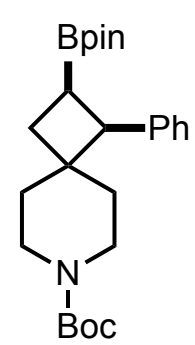

5

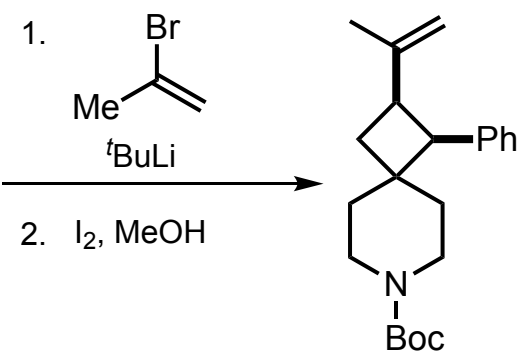

42 
tert-Butyl 1-phenyl-2-(prop-1-en-2-yl)-7-azaspiro[3.5]nonane-7-carboxylate (42): A flamedried 13 x 100 reaction vial under $\mathrm{N}_{2}$ was charged with 2- bromoprop-1-ene (55.0 $\mu \mathrm{L}, 0.615 \mathrm{mmol}$, 4.10 equiv.). THF $(1.5 \mathrm{~mL})$ was added, and the solution cooled to $-78{ }^{\circ} \mathrm{C}$ in a dry ice/acetone bath. $t$-BuLi ( $0.46 \mathrm{~mL}, 1.3 \mathrm{M}$ in pentane, $0.60 \mathrm{mmol}, 4.00$ equiv.) was added dropwise and the solution stirred at $-78{ }^{\circ} \mathrm{C}$ for $30 \mathrm{~min}$. At this time, a solution of Bpin 5 (64 mg, $0.150 \mathrm{mmol}, 1.00$ equiv.) dissolved in THF $(0.50 \mathrm{~mL})$ was added dropwise at $-78^{\circ} \mathrm{C}$ and the mixture was allowed to stir at $-78^{\circ} \mathrm{C}$ for 1 hour. The reaction was then warmed to $-40{ }^{\circ} \mathrm{C}$ by switching to a dry ice/acetonitrile bath and allowed to stir for 1 hour. The reaction was cooled back to $-78{ }^{\circ} \mathrm{C}$ and a solution of $\mathrm{I}_{2}$ (152.0 mg, $0.60 \mathrm{mmol}, 4.00$ equiv.) dissolved in $\mathrm{MeOH}(2.50 \mathrm{~mL})$ was added dropwise down the side of the flask. Upon complete addition, the reaction was allowed to stir at $-78{ }^{\circ} \mathrm{C}$ for 45 minutes then allowed to warm to room temperature and stir for an additional 1 hour. The reaction was quenched upon the addition of $\mathrm{Na}_{2} \mathrm{~S}_{2} \mathrm{O}_{3}$ (sat. solution, $10.0 \mathrm{~mL}$ ) and stirred for another $1 \mathrm{~h}$. The organic layer was separated, and the aqueous phase extracted with EtOAc $(3 \mathrm{x} 15 \mathrm{~mL})$. The combined organic layers were washed with brine $(15 \mathrm{~mL})$, dried over $\mathrm{MgSO}_{4}$, gravity filtered, and concentrated. Crude material was purified by silica gel column chromatography with pentane as the eluent to give 42 as a colorless oil (46.0 mg, 90\% yield). IR (neat): 1688 (s), 1241 (s), 1149 (s) $\mathrm{cm}^{-1}$; ${ }^{1} \mathbf{H}$ NMR (400 MHz, $\left.\mathbf{C D C l}_{3}\right): \delta 7.23-7.15(\mathrm{~m}, 5 \mathrm{H}), 4.86(\mathrm{~s}, 1 \mathrm{H}), 4.77(\mathrm{~s}, 1 \mathrm{H}), 3.58-$ $3.52(\mathrm{~m}, 1 \mathrm{H}), 3.45-3.31(\mathrm{~m}, 3 \mathrm{H}), 3.00(\mathrm{t}, J=5.7 \mathrm{~Hz}, 2 \mathrm{H}), 2.18(\mathrm{t}, J=10.8 \mathrm{~Hz}, 1 \mathrm{H}), 2.10-2.05$ $(\mathrm{m}, 1 \mathrm{H}), 1.84(\mathrm{t}, J=5.7 \mathrm{~Hz}, 2 \mathrm{H}), 1.41(\mathrm{~s}, 9 \mathrm{H}), 1.26(\mathrm{~s}, 3 \mathrm{H}), 1.23-1.17(\mathrm{~m}, 1 \mathrm{H}), 1.14-1.08(\mathrm{~m}$, $1 \mathrm{H}) ;{ }^{13} \mathbf{C}$ NMR (101 MHz, $\left.\mathbf{C D C l}_{3}\right): \delta$ 155.0, 146.2, 139.3, 129.7, 127.6, 126.2, 110.1, 79.3, 52.8, 40.1, 38.3, 37.7, 34.6, 33.8, 28.6, 21.3; HRMS (ESI+): Calculated for $\mathrm{C}_{22} \mathrm{H}_{31} \mathrm{O}_{2} \mathrm{NNa}[\mathrm{M}+\mathrm{Na}]+$ : 364.2247, Found: 364.2249.

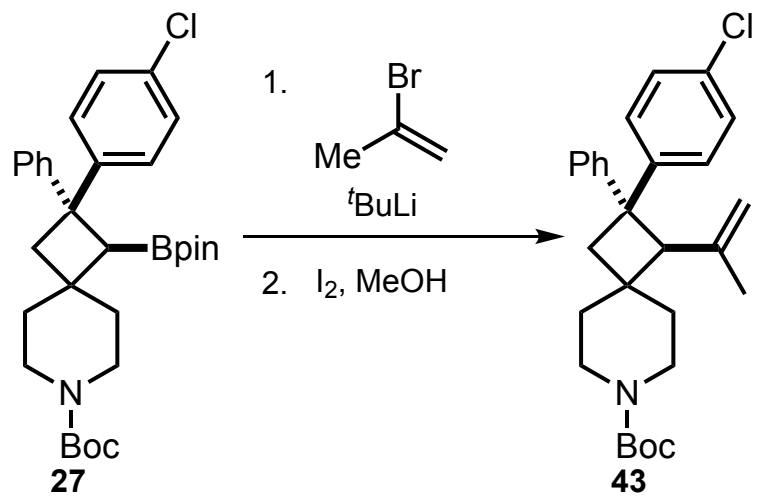

tert-Butyl

2-(4-chlorophenyl)-2-phenyl-1-(prop-1-en-2-yl)-7-azaspiro[3.5]nonane-7carboxylate (43): A flame-dried 13 x 100 reaction vial under $\mathrm{N}_{2}$ was charged with 2- bromoprop- 
1-ene (43.0 $\mu \mathrm{L}, 0.480 \mathrm{mmol}, 4.10$ equiv.). THF (1.5 mL) was added, and the solution cooled to $78^{\circ} \mathrm{C}$ in a dry ice/acetone bath. $t$-BuLi $(0.36 \mathrm{~mL}, 1.3 \mathrm{M}$ in pentane, $0.468 \mathrm{mmol}, 4.00$ equiv. $)$ was added dropwise and the solution stirred at $-78^{\circ} \mathrm{C}$ for $30 \mathrm{~min}$. At this time, a solution of Bpin 27 (63.0 mg, $0.117 \mathrm{mmol}, 1.00$ equiv.) dissolved in THF $(0.50 \mathrm{~mL})$ was added dropwise at $-78{ }^{\circ} \mathrm{C}$ and the mixture was allowed to stir at $-78{ }^{\circ} \mathrm{C}$ for 1 hour. The reaction was then warmed to $-40{ }^{\circ} \mathrm{C}$ by switching to a dry ice/acetonitrile bath and allowed to stir for 1 hour. The reaction was cooled back to $-78^{\circ} \mathrm{C}$ and a solution of $\mathrm{I}_{2}(119.0 \mathrm{mg}, 0.468 \mathrm{mmol}, 4.00$ equiv.) dissolved in $\mathrm{MeOH}(2.50$ $\mathrm{mL}$ ) was added dropwise down the side of the flask. Upon complete addition, the reaction was allowed to stir at $-78{ }^{\circ} \mathrm{C}$ for 45 minutes then allowed to warm to room temperature and stir for an additional 1 hour. The reaction was quenched upon the addition of $\mathrm{Na}_{2} \mathrm{~S}_{2} \mathrm{O}_{3}$ (sat. solution, 10.0 $\mathrm{mL}$ ) and stirred for another $1 \mathrm{~h}$. The organic layer was separated, and the aqueous phase extracted with EtOAc ( $3 \times 15 \mathrm{~mL})$. The combined organic layers were washed with brine $(15 \mathrm{~mL})$, dried over $\mathrm{MgSO}_{4}$, gravity filtered, and concentrated. Crude material was purified by silica gel column chromatography (Gradient: hexane to $15 \% \mathrm{Et}_{2} \mathrm{O}$ :hexanes) to obtain 43 as a colorless oil (41.0 $\mathrm{mg}$, 78\% yield). IR (neat): 1686 (s), 1423 (w), 1145 (s), 731 (w) $\mathrm{cm}^{-1} ;{ }^{1}$ H NMR (400 MHz, CDCl $)$ ): $\delta 7.36-7.34(\mathrm{~m}, 2 \mathrm{H}), 7.30-7.26(\mathrm{~m}, 2 \mathrm{H}), 7.18-7.10(\mathrm{~m}, 5 \mathrm{H}), 4.96(\mathrm{~s}, 1 \mathrm{H}), 4.55(\mathrm{~s}, 1 \mathrm{H}), 3.69$ (s, 1H), $3.66-3.55(\mathrm{~m}, 2 \mathrm{H}), 3.13-3.02(\mathrm{~m}, 2 \mathrm{H}), 2.98(\mathrm{~d}, J=12.0 \mathrm{~Hz}, 1 \mathrm{H}), 2.68(\mathrm{dd}, J=12.1,2.0$ $\mathrm{Hz}, 1 \mathrm{H}), 1.64-1.61(\mathrm{~m}, 1 \mathrm{H}), 1.59(\mathrm{~s}, 3 \mathrm{H}), 1.56-1.47(\mathrm{~m}, 2 \mathrm{H}), 1.45(\mathrm{~s}, 9 \mathrm{H}), 1.42$ - $1.39(\mathrm{~m}$, 1H); ${ }^{13} \mathbf{C}$ NMR (101 MHz, $\left.\mathbf{C D C l}_{3}\right): \delta$ 155.0, 152.3, 145.3, 142.1, 131.3, 128.8, 128.7, 128.1, 125.9, 125.6, 117.5, 79.4, 61.8, 50.0, 41.5, 39.1, 37.5, 33.7, 28.6, 24.8; HRMS (ESI+): Calculated for $\mathrm{C}_{28} \mathrm{H}_{34} \mathrm{O}_{2} \mathrm{NClNa}[\mathrm{M}+\mathrm{Na}]+:$ 474.2170, Found: 474.2169.
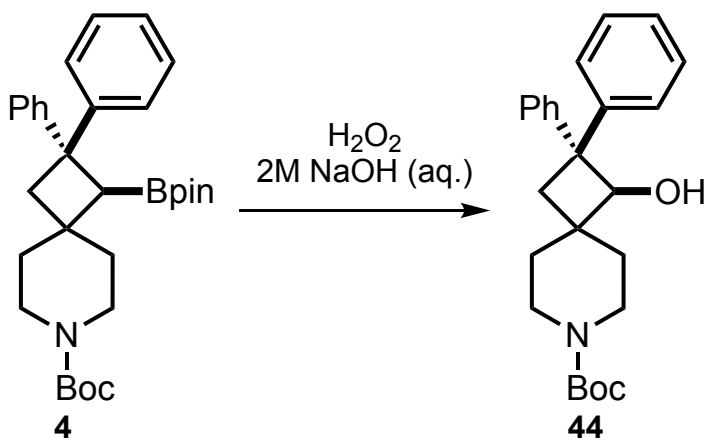

tert-Butyl 1-hydroxy-2,2-diphenyl-7-azaspiro[3.5]nonane-7-carboxylate (44): Bpin 4 (50.0 $\mathrm{mg}, 0.099 \mathrm{mmol}, 1.0$ equiv.) was taken in $2.0 \mathrm{~mL} \mathrm{THF}$, cooled to $0{ }^{\circ} \mathrm{C}$ (ice bath) followed by the addition of $1.0 \mathrm{~mL} 2 \mathrm{M} \mathrm{NaOH}$ (aq.) and $0.5 \mathrm{~mL} \mathrm{H} \mathrm{O}_{2}$. After $1 \mathrm{~h}, 10 \mathrm{~mL}$ water was added, and the aqueous layer was extracted with EtOAc $(3 \times 10 \mathrm{~mL})$. Combined organic layer was washed with 
brine $(10 \mathrm{~mL})$, dried over $\mathrm{Na}_{2} \mathrm{SO}_{4}$ and concentrated in reduced pressure to obtain 44 as a clear oil (35.0 mg, $0.089 \mathrm{mmol}, 90 \%$ isolated yield). IR (neat): 3417 (w), 1661 (s), 1149 (s), 704 (s) cm ${ }^{-1}$; ${ }^{1}$ H NMR (400 MHz, CDCl 3$): \delta 7.42-7.39(\mathrm{~m}, 2 \mathrm{H}), 7.33-7.22(\mathrm{~m}, 6 \mathrm{H}), 7.17$ (t, J= $\left.7.3 \mathrm{~Hz}, 1 \mathrm{H}\right)$, $7.12-7.08(\mathrm{~m}, 1 \mathrm{H}), 4.65(\mathrm{~d}, J=10.5 \mathrm{~Hz}, 1 \mathrm{H}), 3.68-3.65(\mathrm{~m}, 2 \mathrm{H}), 3.17-3.01(\mathrm{~m}, 3 \mathrm{H}), 2.00(\mathrm{~d}$, $J=12.4 \mathrm{~Hz}, 1 \mathrm{H}), 1.83(\mathrm{~d}, J=10.8 \mathrm{~Hz}, 1 \mathrm{H}), 1.69-1.62(\mathrm{~m}, 1 \mathrm{H}), 1.50-1.45(\mathrm{~m}, 2 \mathrm{H}), 1.42(\mathrm{~s}, 9 \mathrm{H})$, 1.36 - 1.25 (m, 1H); ${ }^{13} \mathbf{C}$ NMR (101 $\left.\mathbf{M H z}, \mathbf{C D C l}_{3}\right): \delta 155.0,151.3,141.4,128.9,128.6,128.4$, 126.6, 126.0, 125.8, 80.5, 79.5, 54.2, 41.3, 38.2, 36.8, 29.8, 28.6; HRMS (ESI+): Calculated for $\mathrm{C}_{25} \mathrm{H}_{31} \mathrm{O}_{3} \mathrm{NNa}[\mathrm{M}+\mathrm{Na}]+:$ 416.2196, Found: 416.2197.

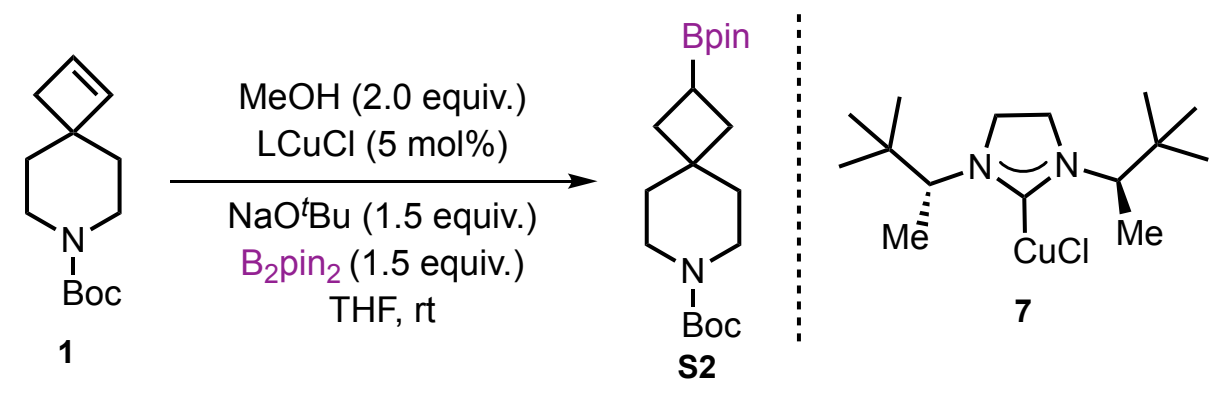

\section{tert-butyl}

2-(4,4,5,5-tetramethyl-1,3,2-dioxaborolan-2-yl)-7-azaspiro[3.5]nonane-7-

carboxylate (S2): The compound was prepared following the general procedure A. MeOH (16.0 $\mu \mathrm{L}, 0.40$ mmol, 2.0 equiv.) was used as electrophile. Purification by silica-gel column chromatography (Gradient: hexane to 20\% EtOAc:hexanes) yields protoboration product S2 (54 $\mathrm{mg}, 77 \%$ isolated yield) as white solid. This Bpin was oxidized to corresponding alcohol to confirm the regioselectivity.

IR (neat): 1687 (s), 1242 (s), 1144 (s) cm ${ }^{-1} ;{ }^{1} \mathbf{H}$ NMR (400 MHz, CDCl $): \delta 3.30-3.27$ (m, 2H), $3.25-3.22(\mathrm{~m}, 2 \mathrm{H}), 1.89-1.83(\mathrm{~m}, 2 \mathrm{H}), 1.82-1.73(\mathrm{~m}, 3 \mathrm{H}), 1.55-1.52(\mathrm{~m}, 2 \mathrm{H}), 1.47-1.44$ (m, 2H), 1.42 (s, 9H), 1.22 (s, 12H); ${ }^{13} \mathbf{C}$ NMR (126 MHz, $\left.\mathbf{C D C l}_{3}\right): \delta$ 155.1, 83.2, 79.2, 38.1, 37.2, 36.7, 33.1, 28.6, 24.8; HRMS (ESI+): Calculated for $\mathrm{C}_{19} \mathrm{H}_{34} \mathrm{O}_{4} \mathrm{NBNa}[\mathrm{M}+\mathrm{Na}]+$ : 374.2473 , Found: 374.2474. 


\section{Single crystal X-ray diffraction analysis of 26:}

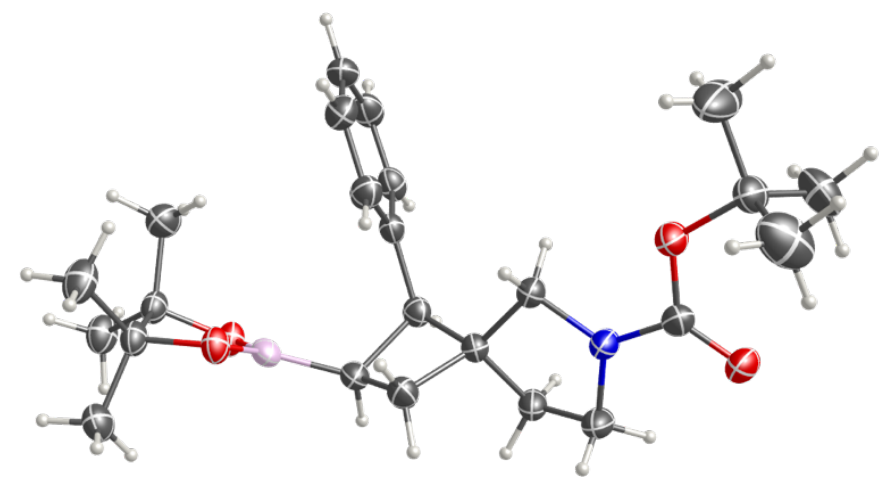

Table S1 Crystal data and structure refinement for 26.

Identification code

Empirical formula

Formula weight

Temperature/K

Crystal system

Space group

Volume

$\mathrm{Z}$

$\rho_{\text {calc }}$

$\mu$

$\mathrm{F}(000)$

Crystal size

Radiation

$2 \Theta$ range for data collection

Index ranges

Reflections collected

Independent reflections

Data/restraints/parameters

Goodness-of-fit on $\mathrm{F}^{2}$

Final $\mathrm{R}$ indexes $[\mathrm{I}>=2 \sigma(\mathrm{I})]$

Final $\mathrm{R}$ indexes [all data]

Largest diff. peak/hole
26

$\mathrm{C}_{24} \mathrm{H}_{36} \mathrm{BNO}_{4}$

413.35

173(2)

triclinic

$\mathrm{P}-1$

$\mathrm{a}=9.9066(4) \AA$.

$\mathrm{b}=11.0766(4) \AA$

$\mathrm{c}=11.6203(5) \AA$

$1171.31(8) \AA^{3}$

2

$1.172 \mathrm{~g} / \mathrm{cm}^{3}$

$0.078 \mathrm{~mm}^{-1}$

448.0

$0.301 \times 0.175 \times 0.143$

$\mathrm{mm}^{3}$

$\operatorname{MoK} \alpha(\lambda=0.71073)$

3.812 to $52.82^{\circ}$

$-12 \leq \mathrm{h} \leq 12,-13 \leq \mathrm{k} \leq 13,-14 \leq 1 \leq 14$

51225

$4810\left[R_{\text {int }}=0.0835, R \sigma=0.0360\right]$

$4810 / 0 / 278$

1.021

$R_{1}=0.0458, \omega R_{2}=0.1031$

$R_{1}=0.0740, \omega R_{2}=0.1180$

$0.21 /-0.21$ e $\AA^{-3}$ 


\section{Single crystal X-ray diffraction analysis of 41:}

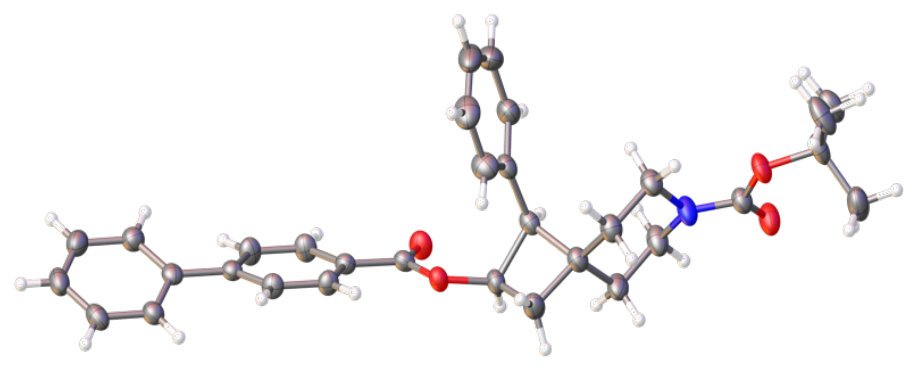

Table S2 Crystal data and structure refinement for 41.

Identification code

Empirical formula

Formula weight

Temperature/K

Crystal system

Space group

Volume

Z

$\rho_{\text {calc }}$

$\mu$

$\mathrm{F}(000)$

Crystal size

Radiation

$2 \Theta$ range for data collection

Index ranges

Reflections collected

Independent reflections

Data/restraints/parameters

Goodness-of-fit on $\mathrm{F}^{2}$

Final $R$ indexes $[\mathrm{I}>=2 \sigma(\mathrm{I})]$

Final $\mathrm{R}$ indexes [all data]

Largest diff. peak/hole
41

$\mathrm{C}_{32} \mathrm{H}_{35} \mathrm{NO}_{4}$

497.61

153.0

triclinic

P-1

$a=10.956(5) \AA$

$\mathrm{b}=11.975(5) \AA$

$\mathrm{c}=22.501(9) \AA$

2684.6(19) $\AA^{3}$

4

$1.231 \mathrm{~g} / \mathrm{cm}^{3}$

$0.080 \mathrm{~mm}^{-1}$

1064.0

$0.17 \times 0.09 \times 0.06 \mathrm{~mm}^{3}$

$\operatorname{MoK} \alpha(\lambda=0.71073)$

3.676 to $52.794^{\circ}$

$-13 \leq \mathrm{h} \leq 13,-14 \leq \mathrm{k} \leq 14,-28 \leq 1 \leq 28$

135959

$10950\left[R_{\text {int }}=0.0780, R \sigma=0.0301\right]$

$10950 / 0 / 673$

1.032

$R_{1}=0.0463, \mathrm{w} R_{2}=0.1089$

$R_{1}=0.0672, \mathrm{w} R_{2}=0.1215$

$0.45 /-0.22$ e $\AA^{-3}$ $\alpha=86.546(10)^{\circ}$

$\beta=79.404(7)^{\circ}$

$\gamma=67.694(10)^{\circ}$ 


\section{Spectra}
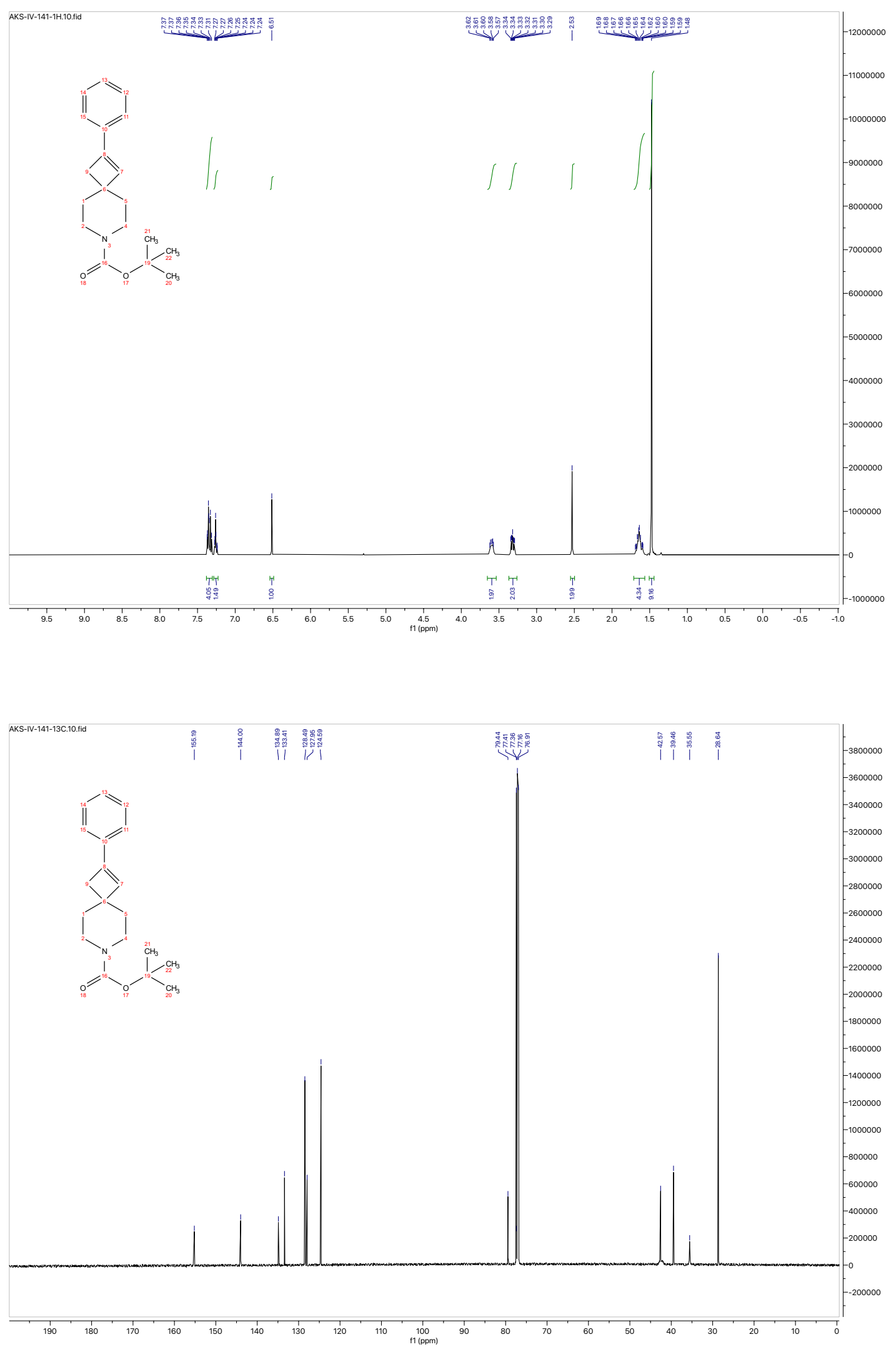

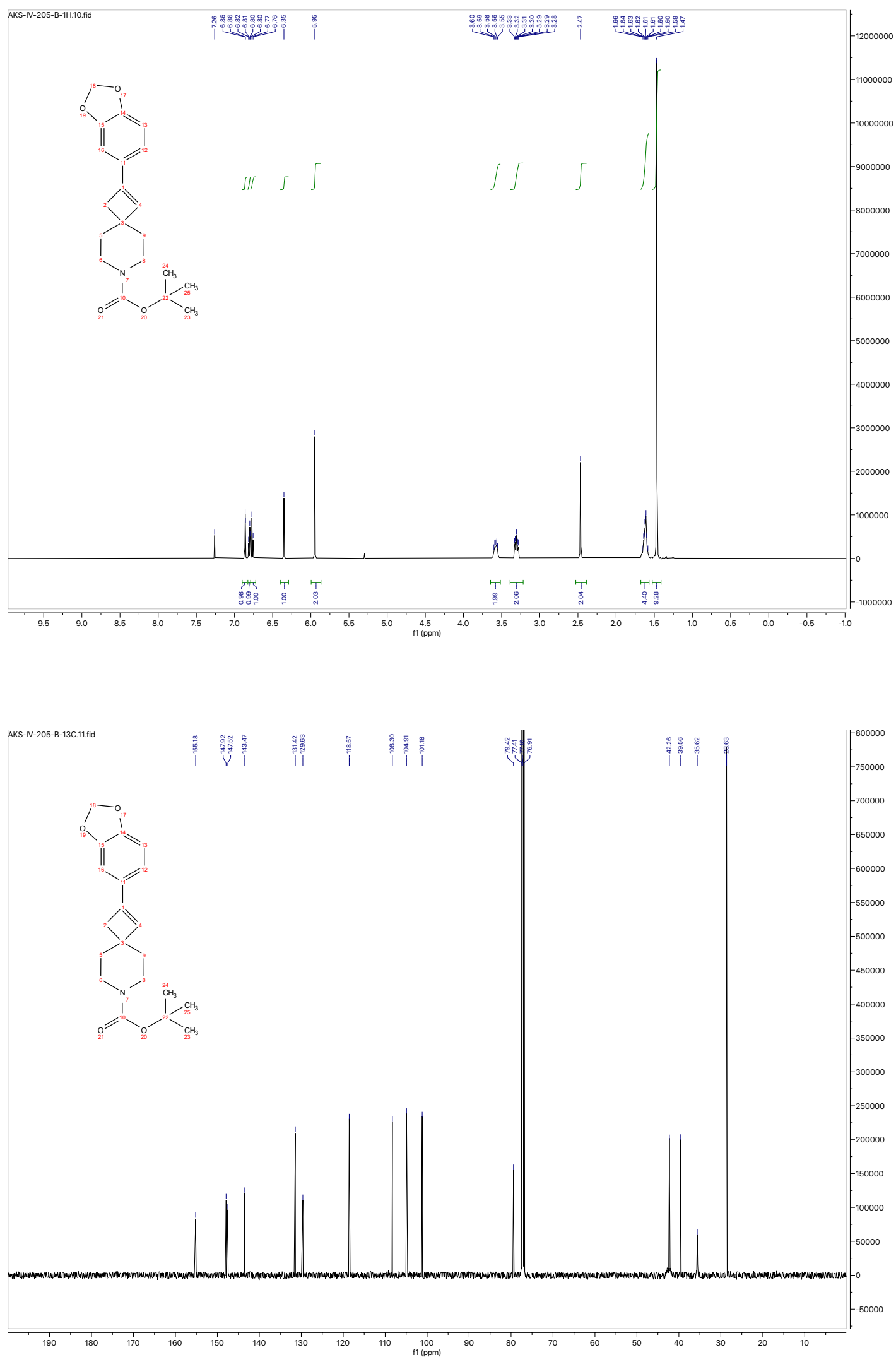

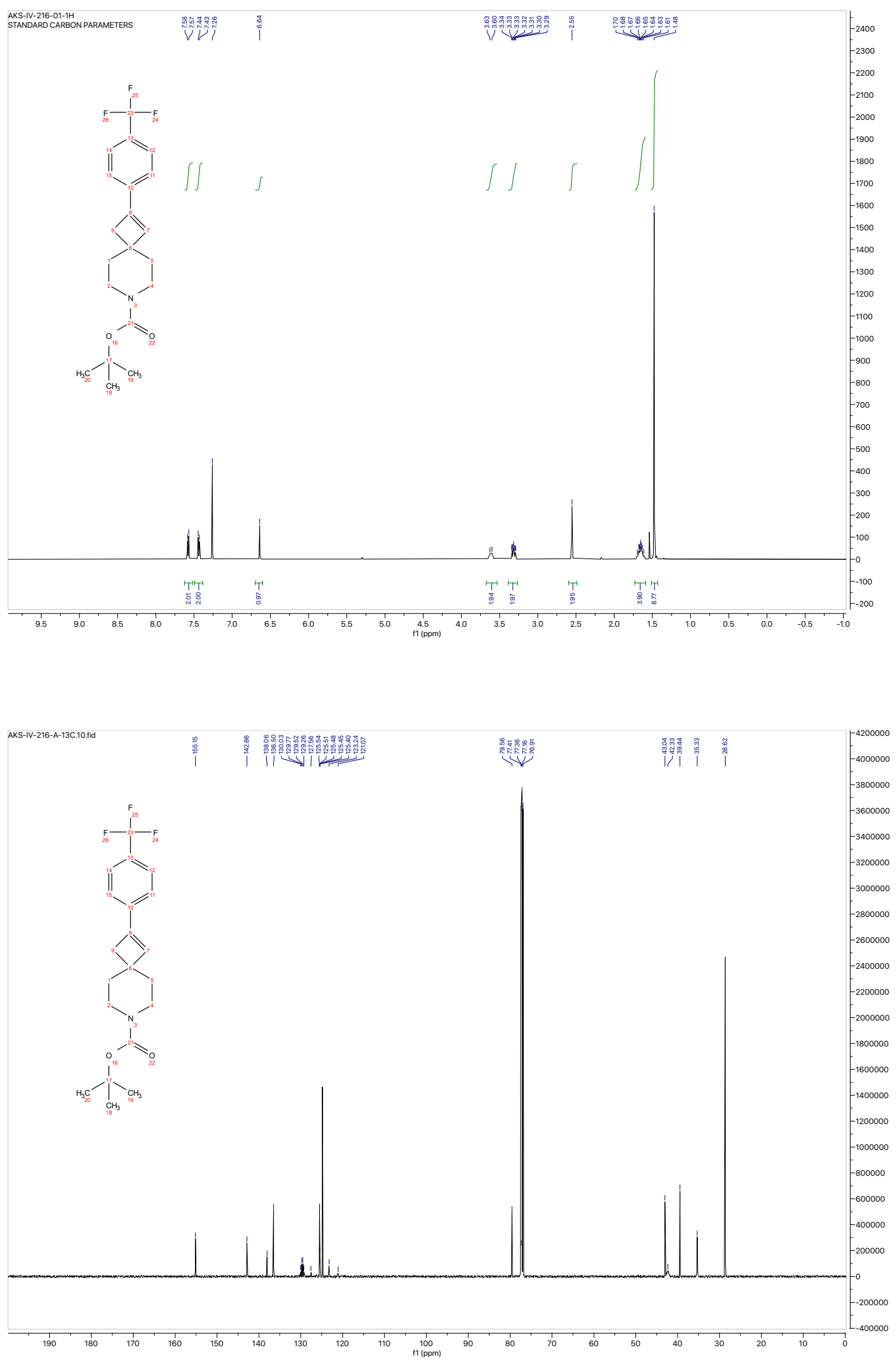
Arylboration of Spirocyclic Cyclobutenes

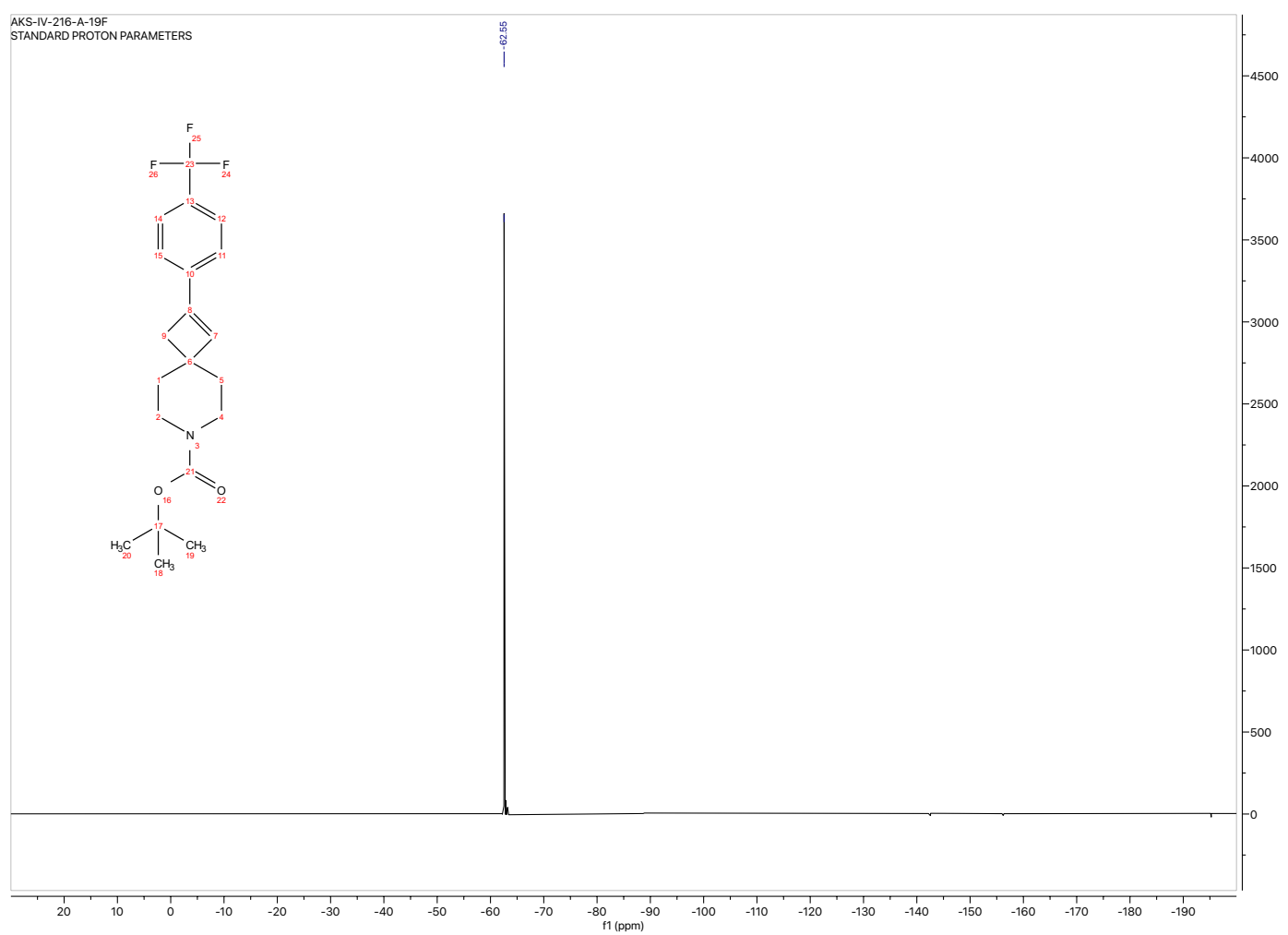


Arylboration of Spirocyclic Cyclobutenes
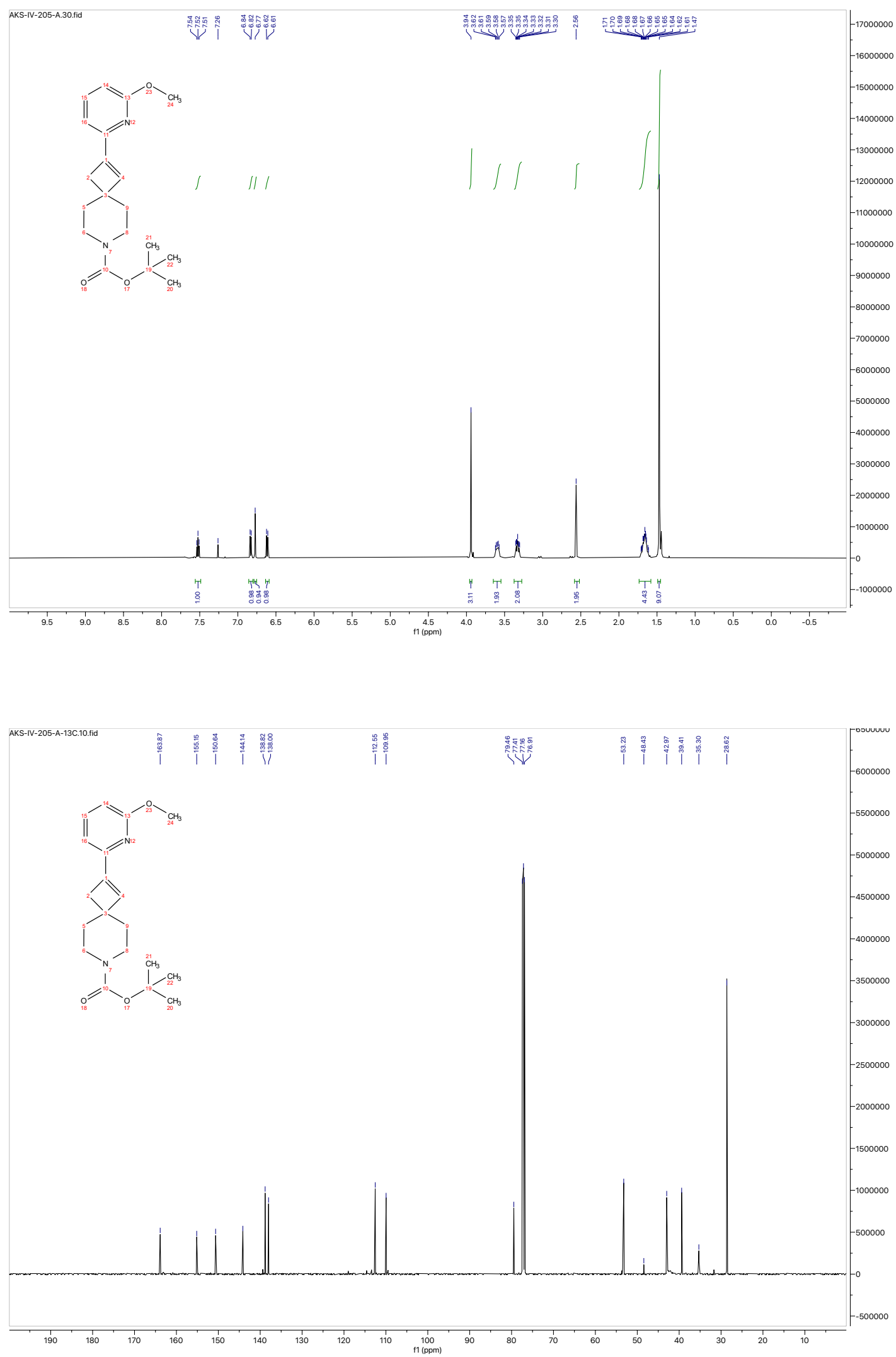
Arylboration of Spirocyclic Cyclobutenes
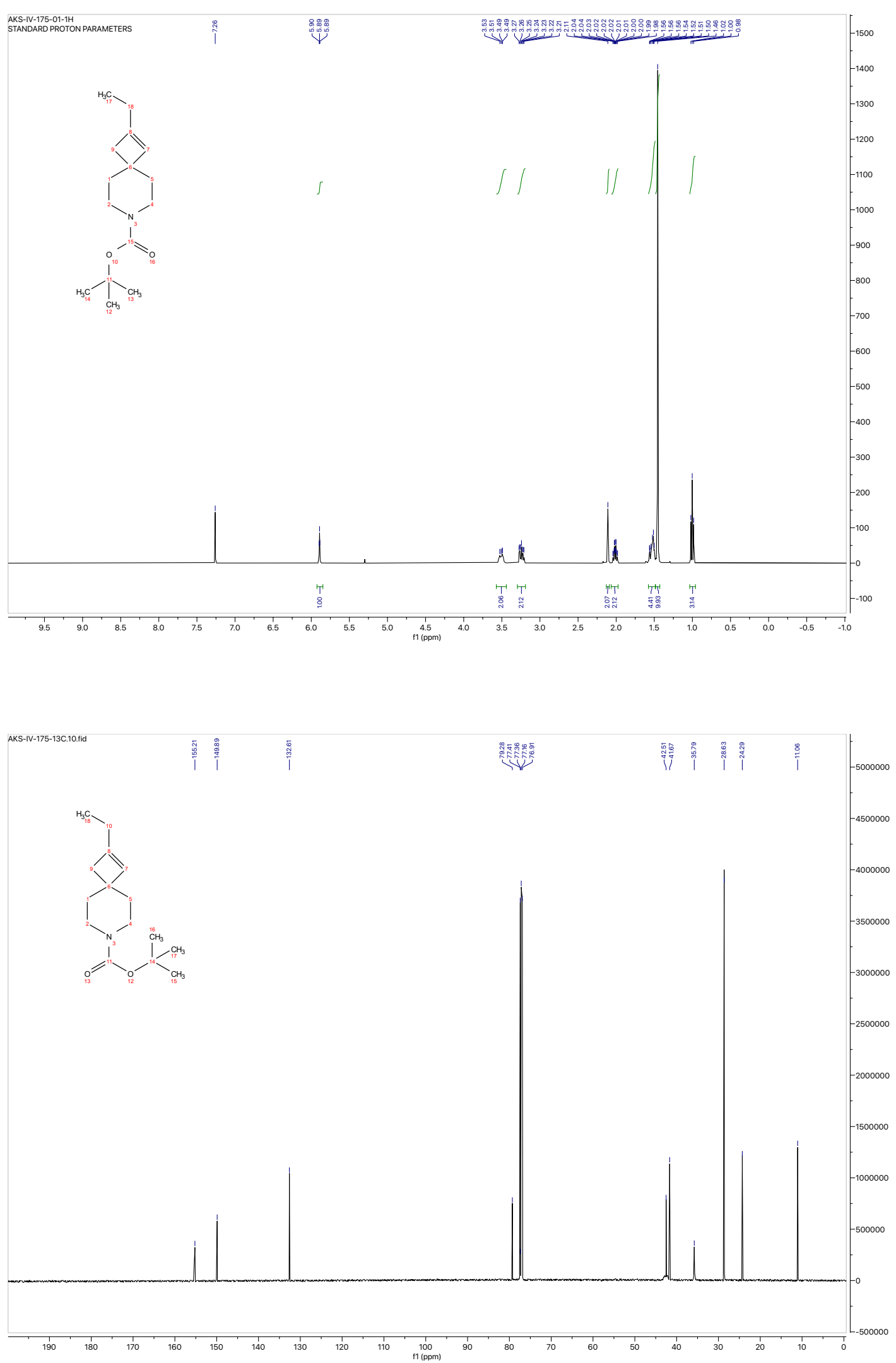

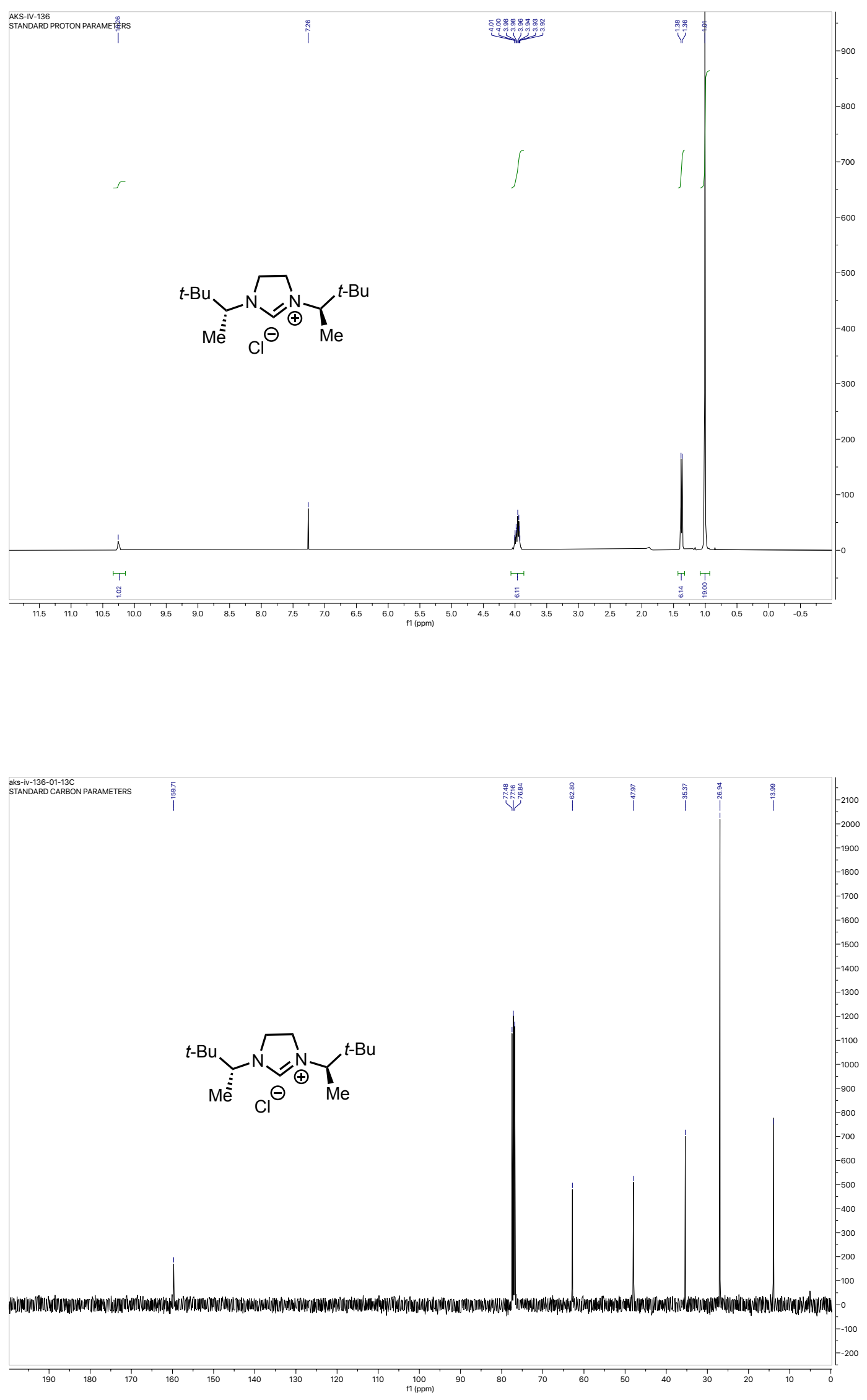

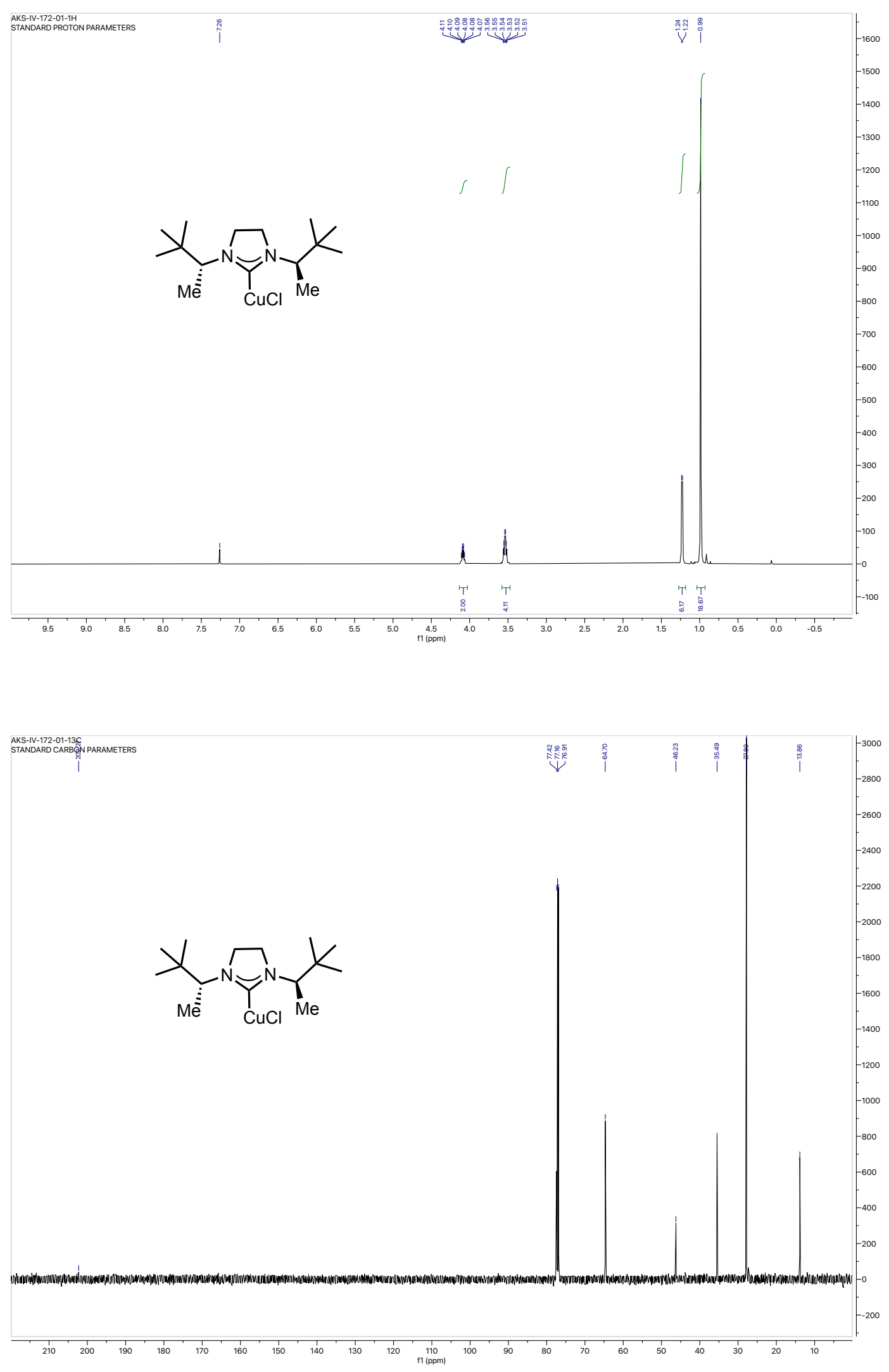

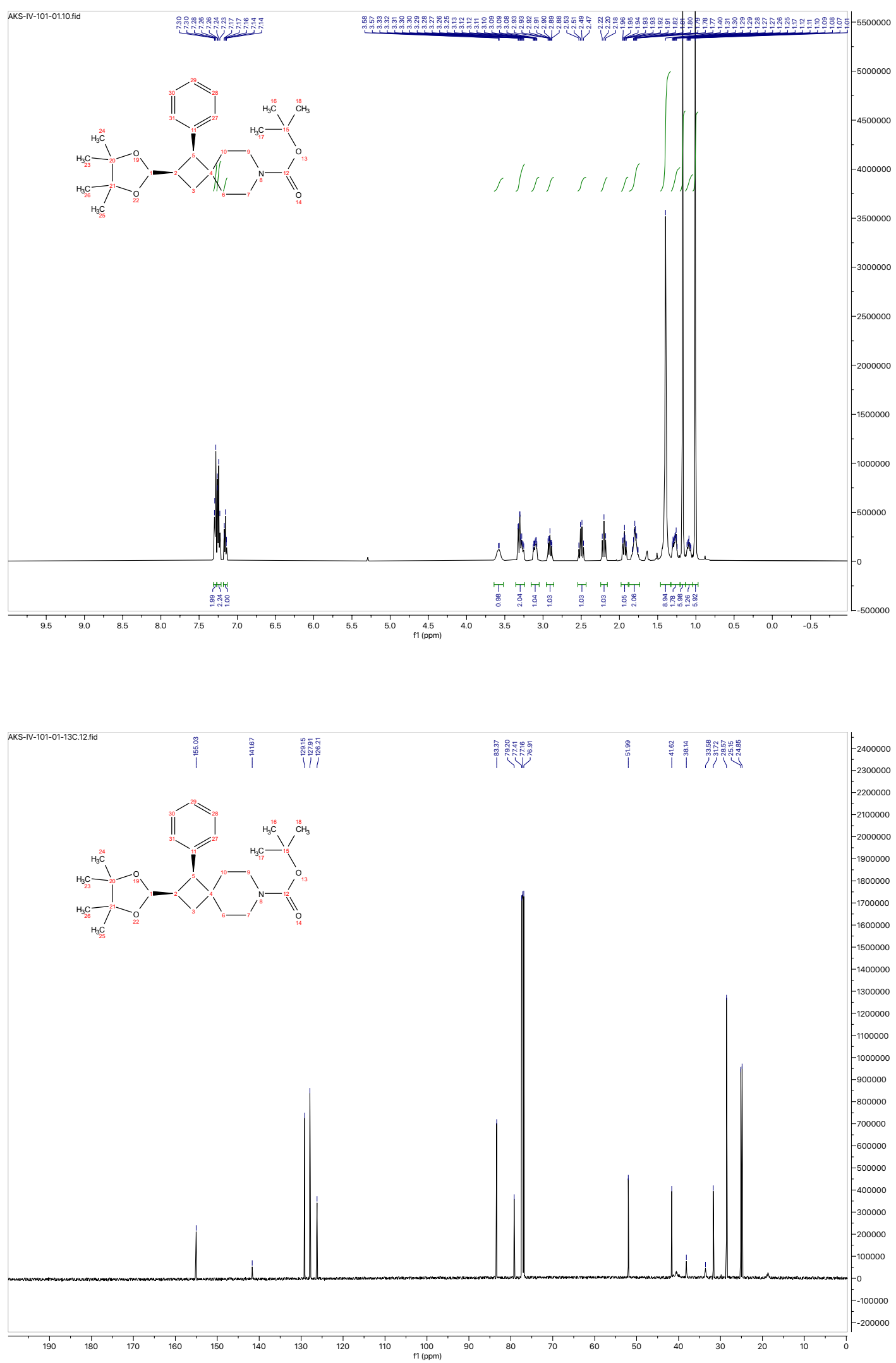
Arylboration of Spirocyclic Cyclobutenes

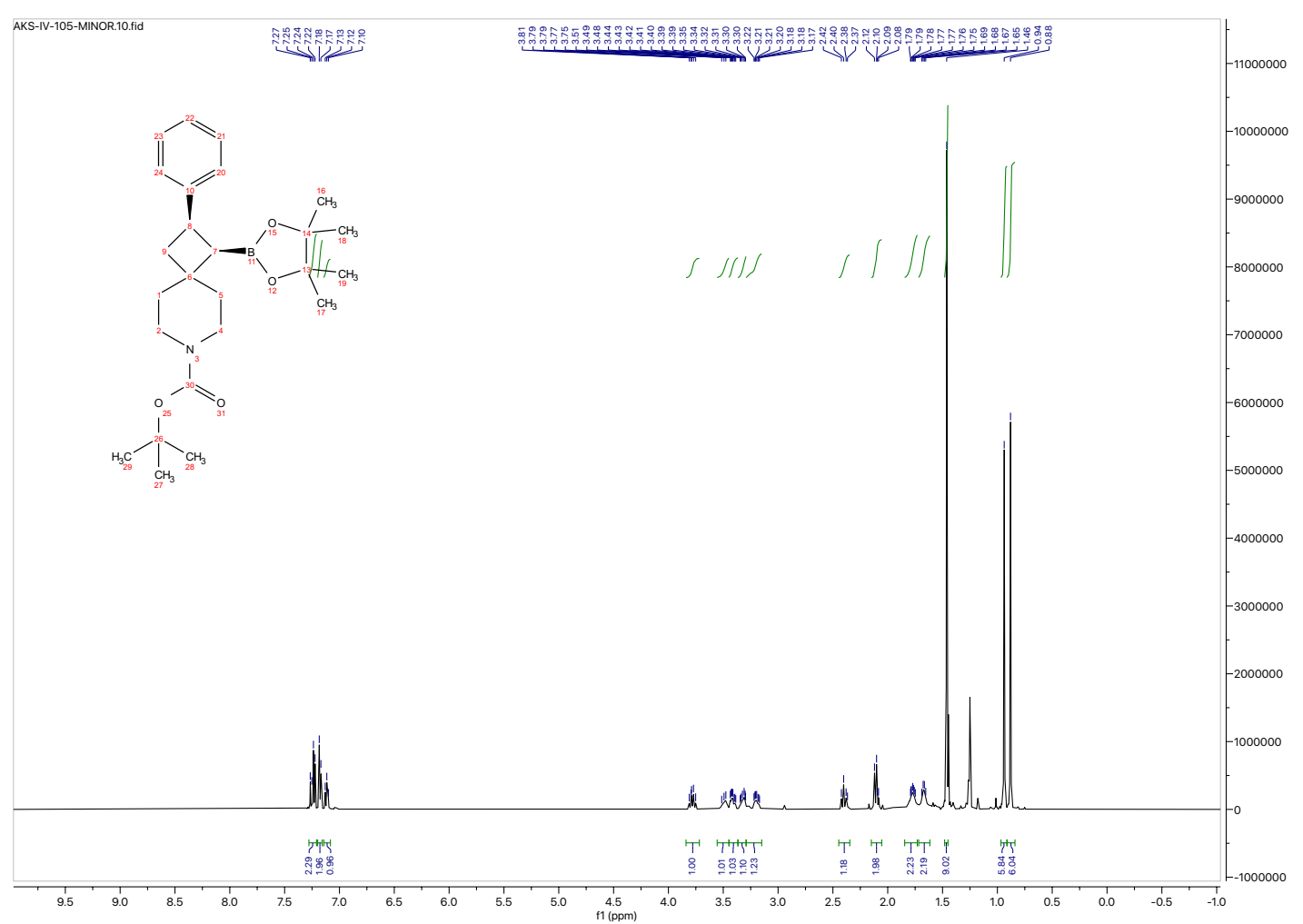

Minor regioisomer 

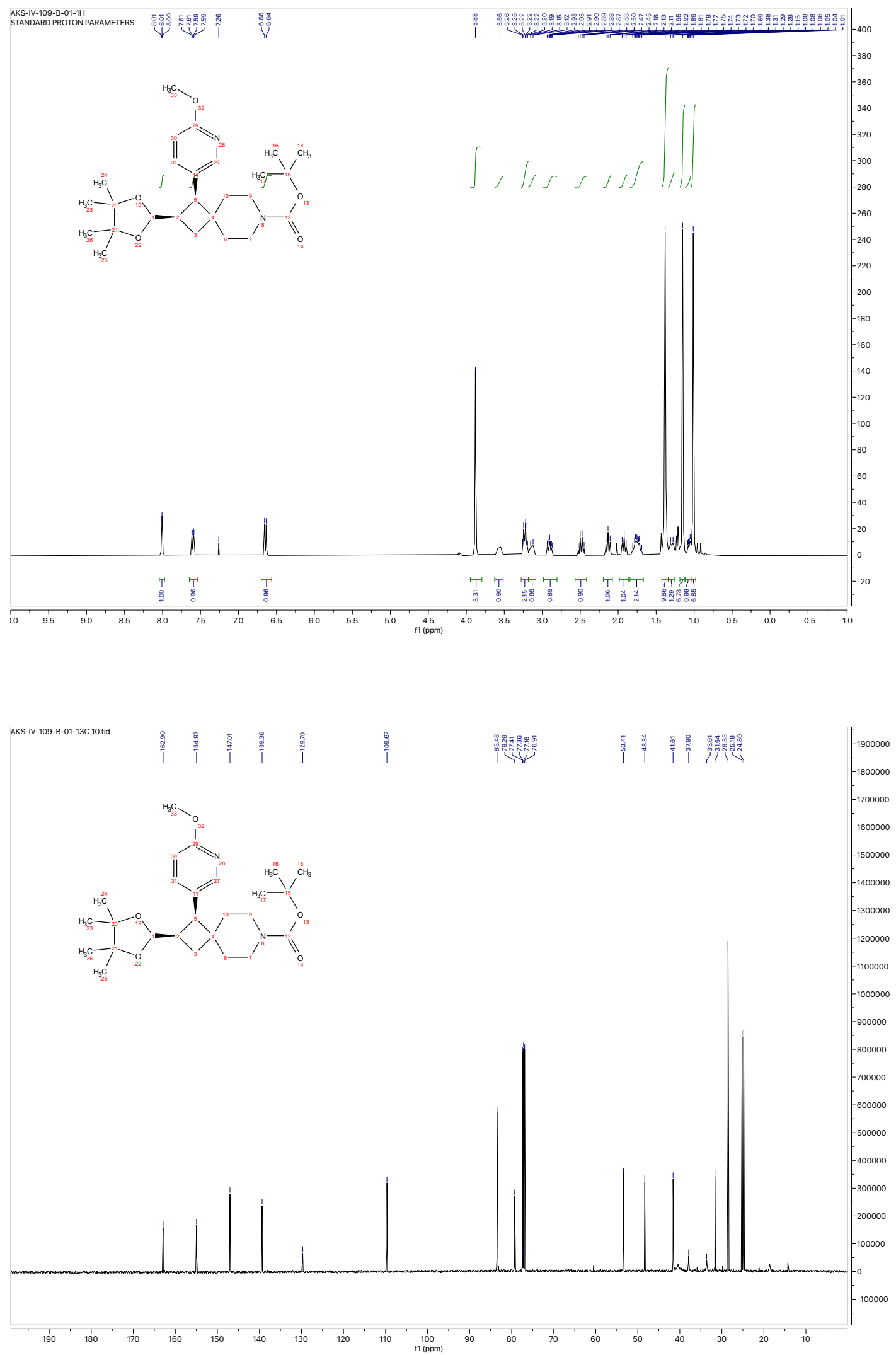

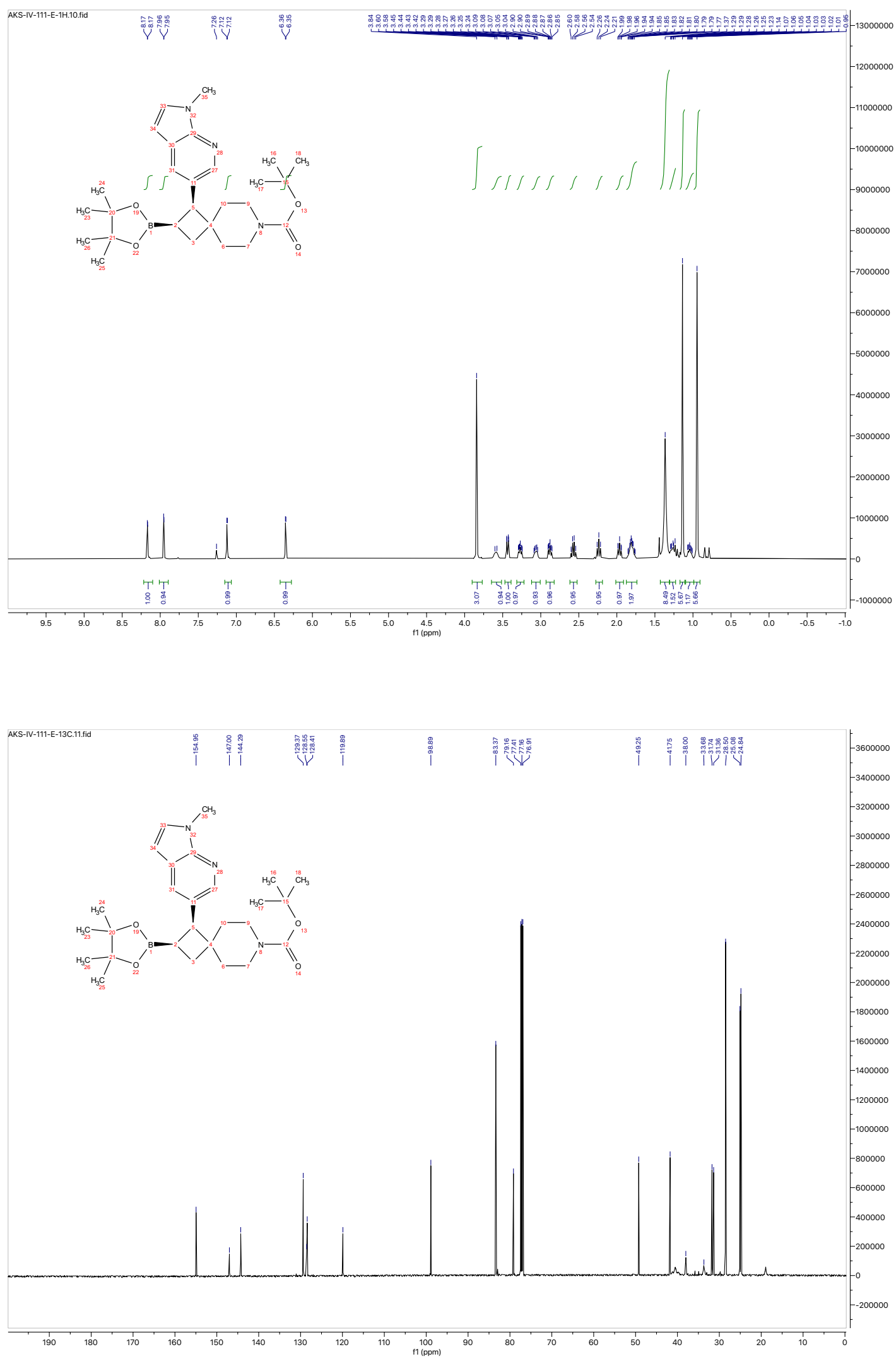

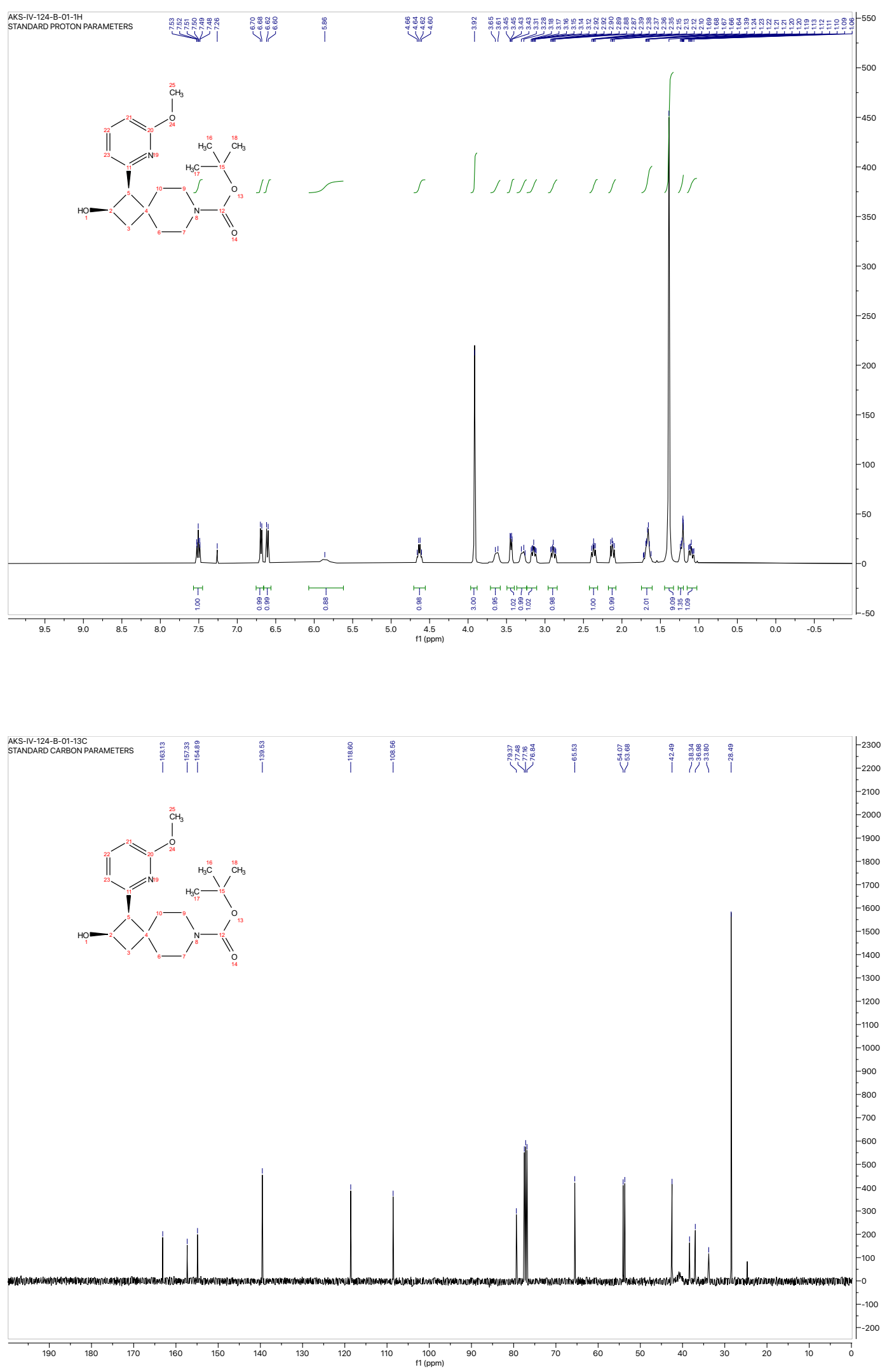

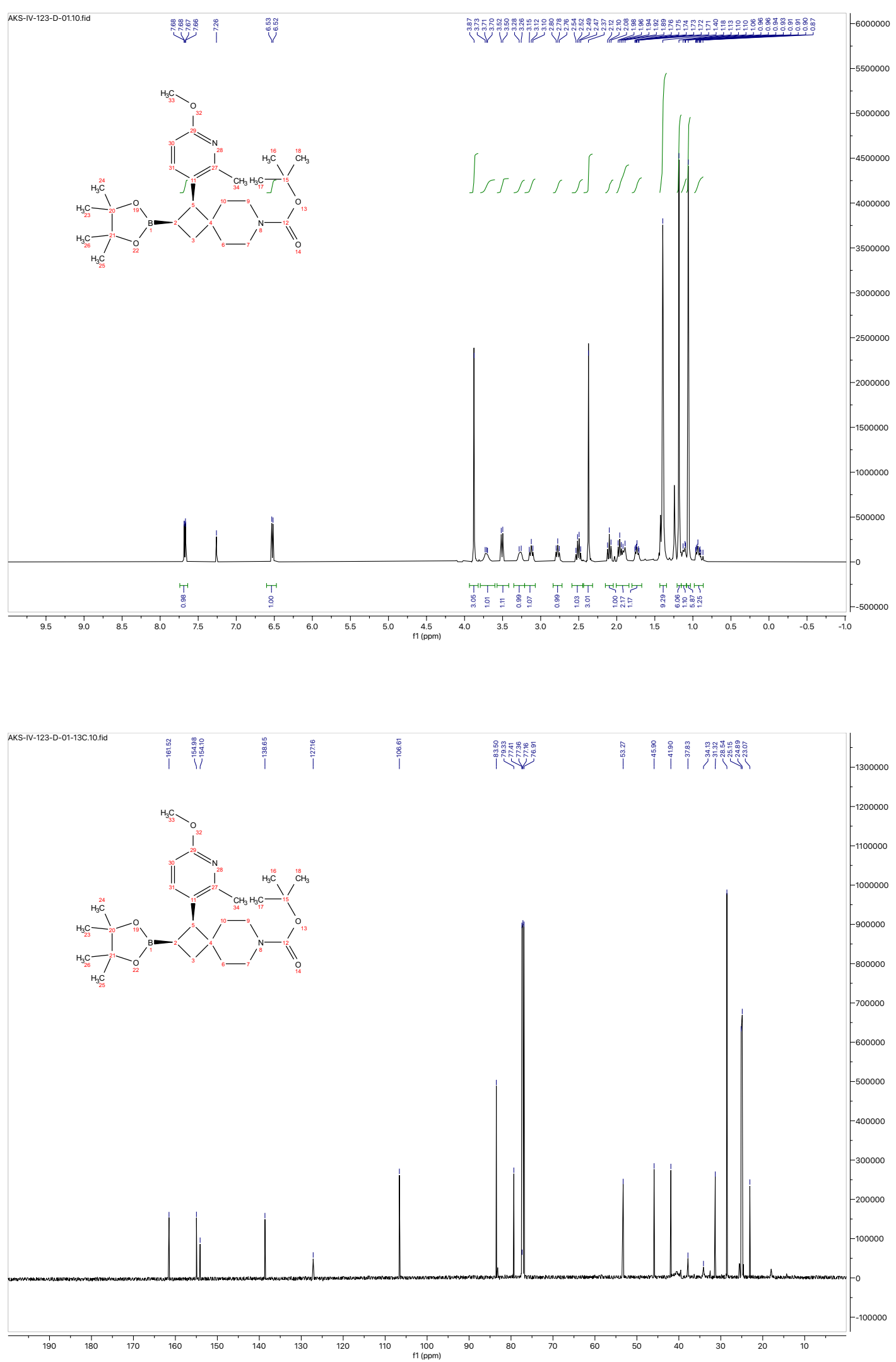

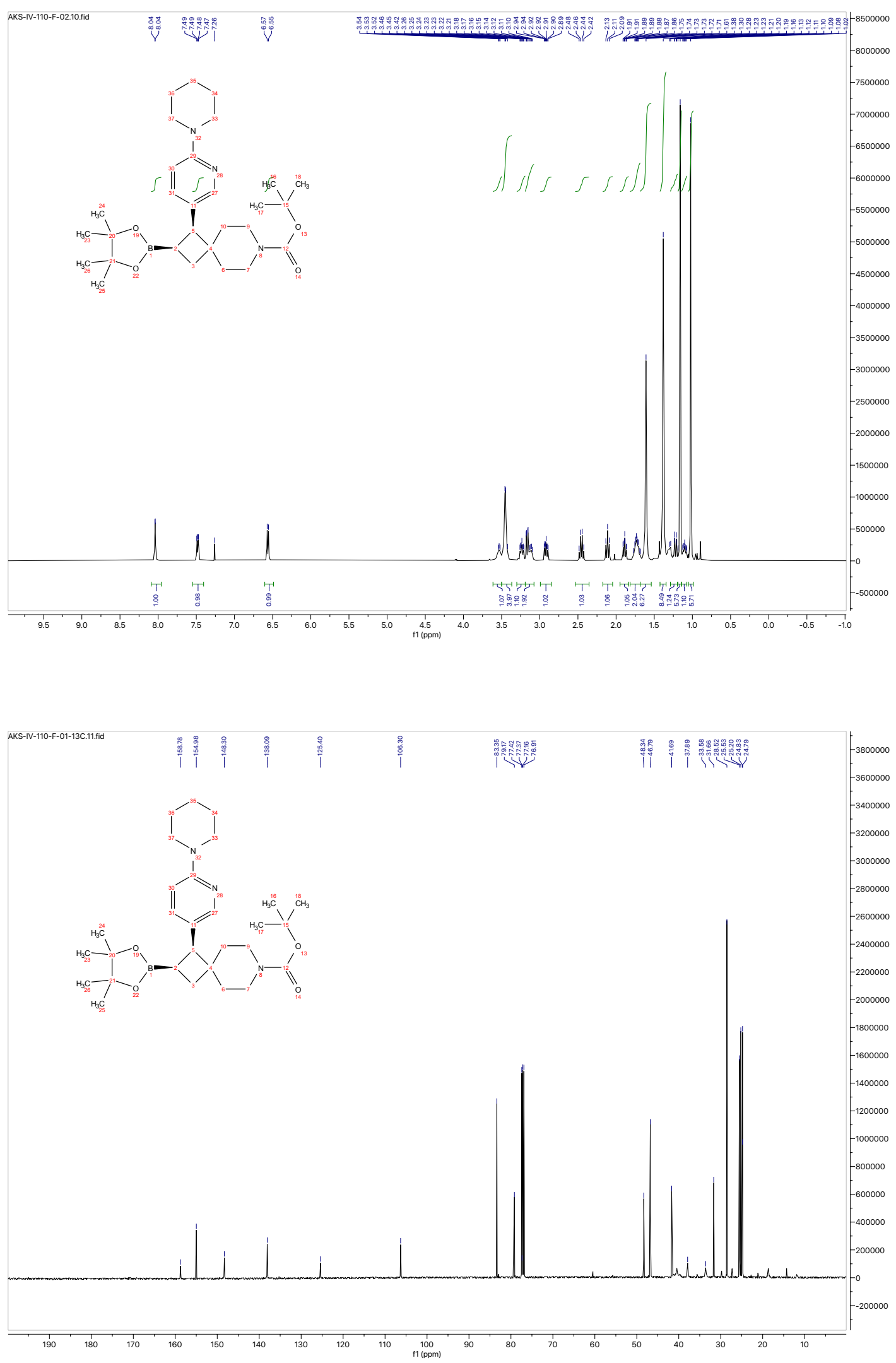

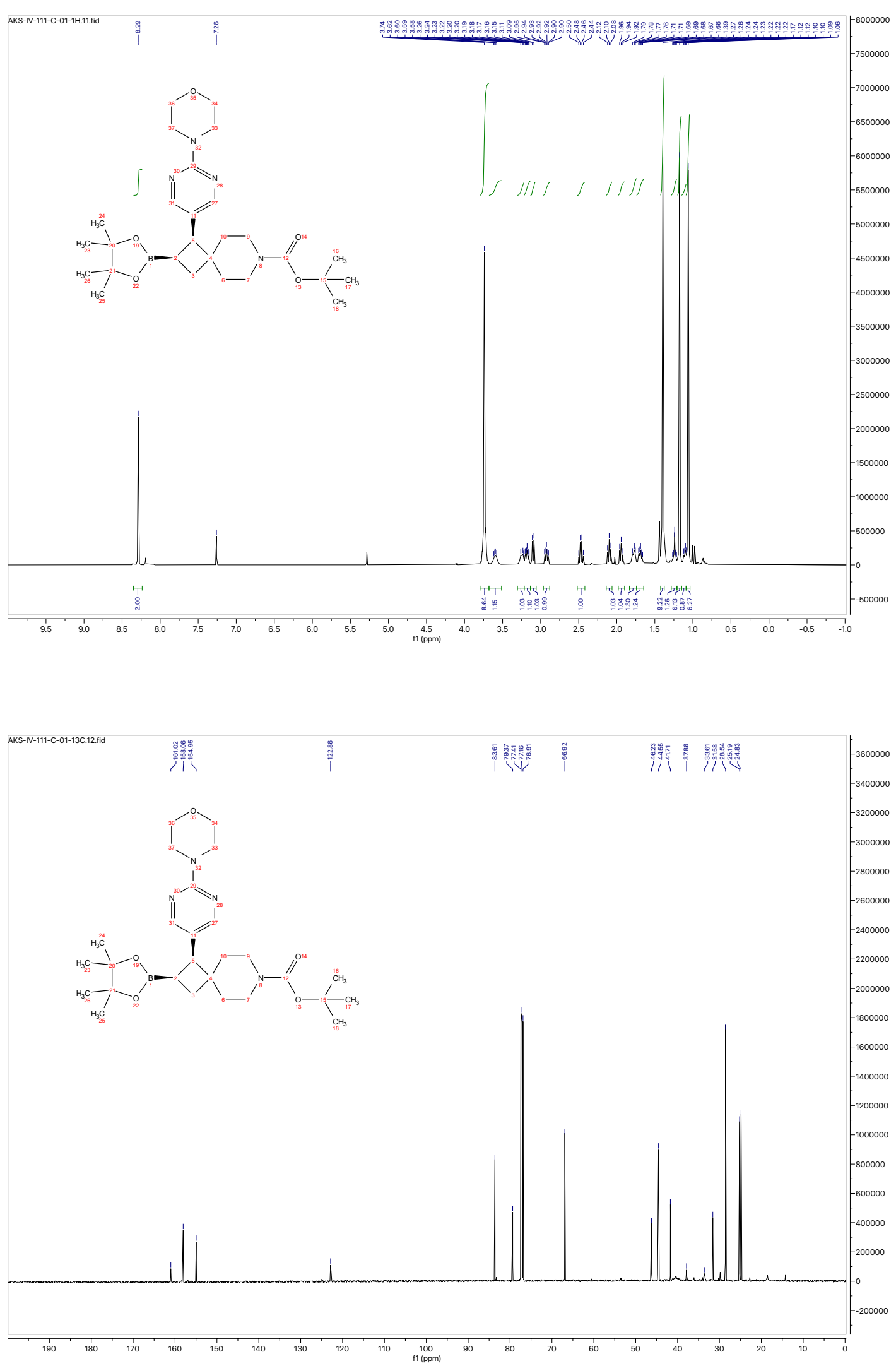

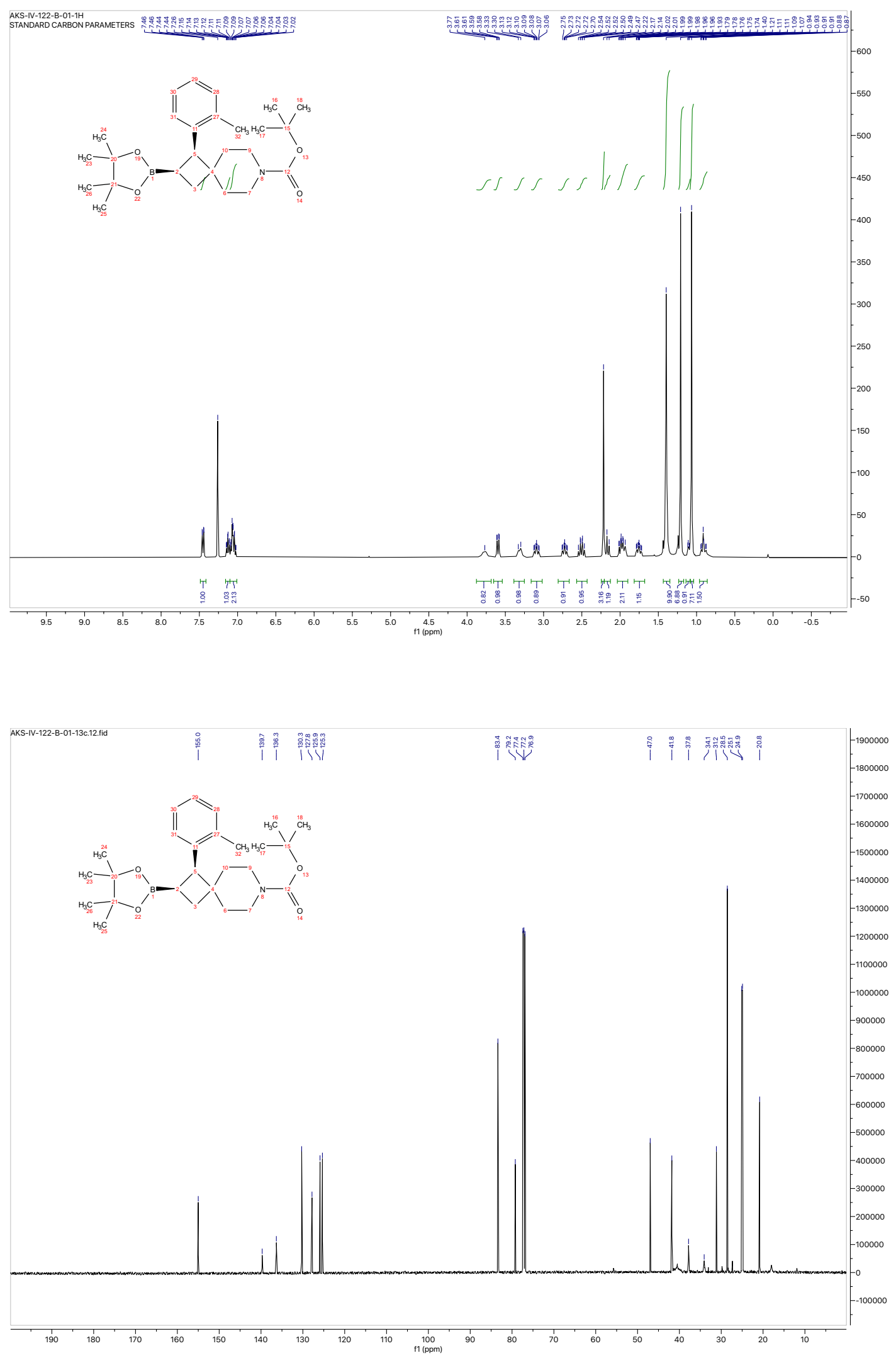

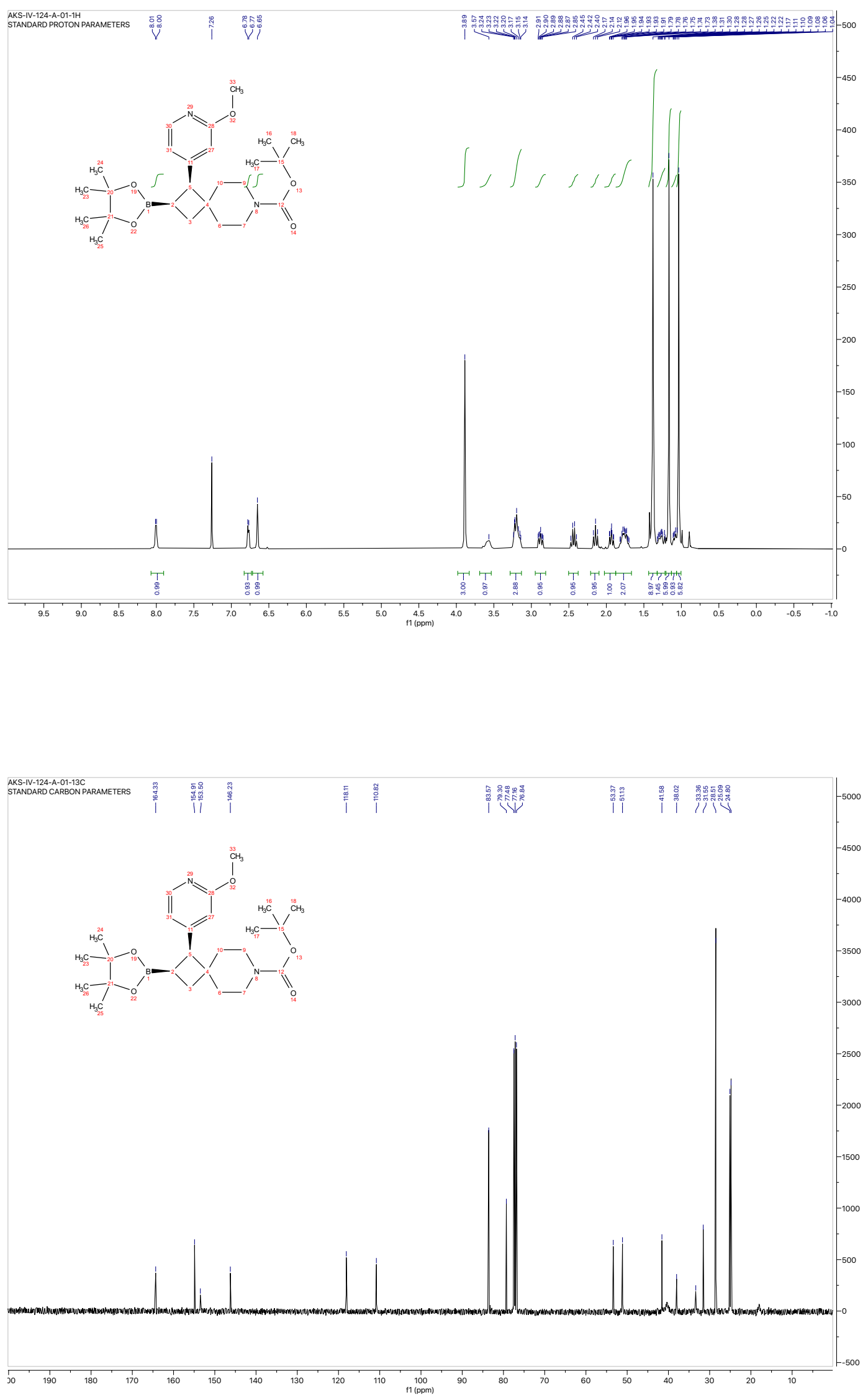

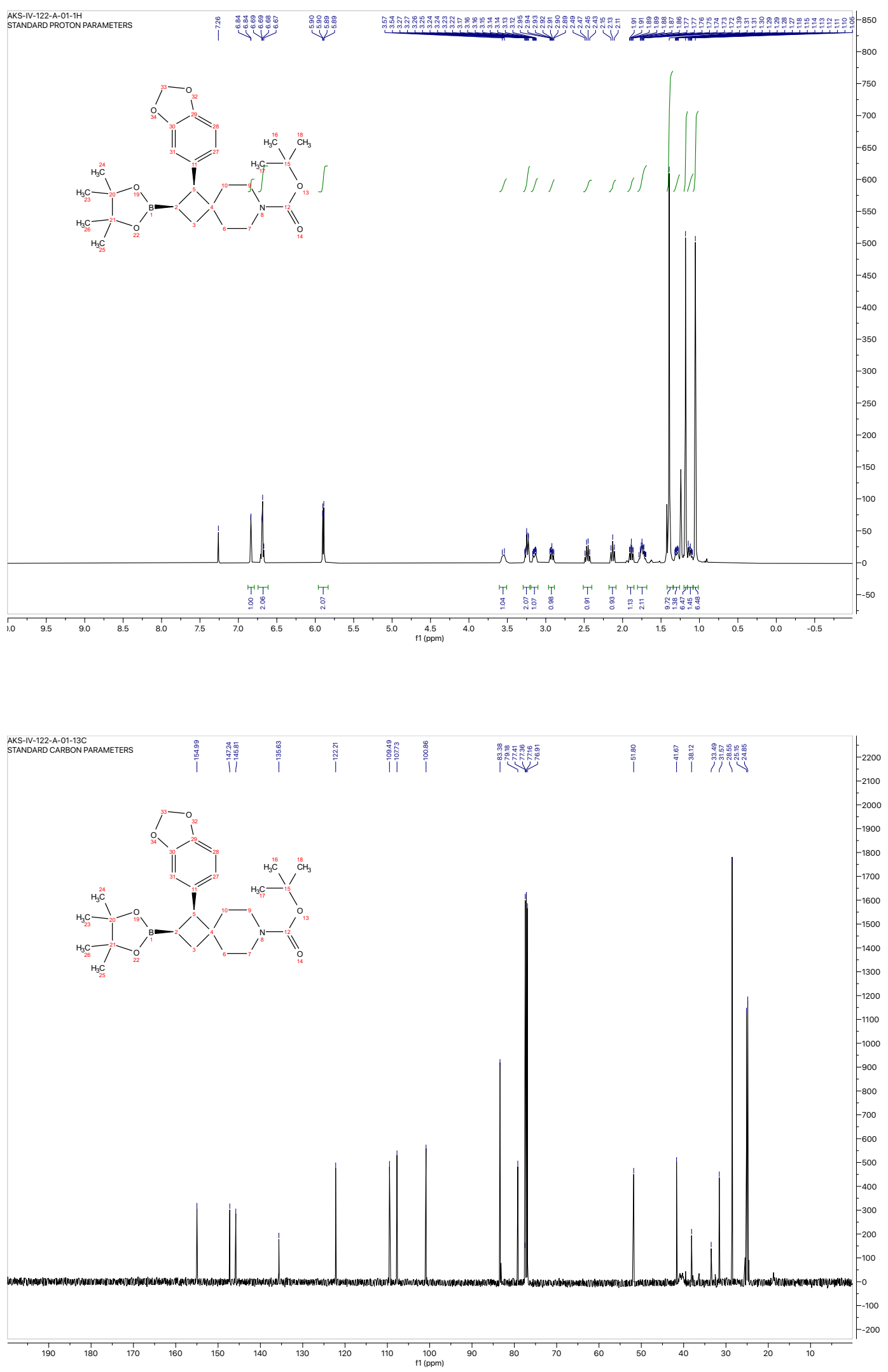

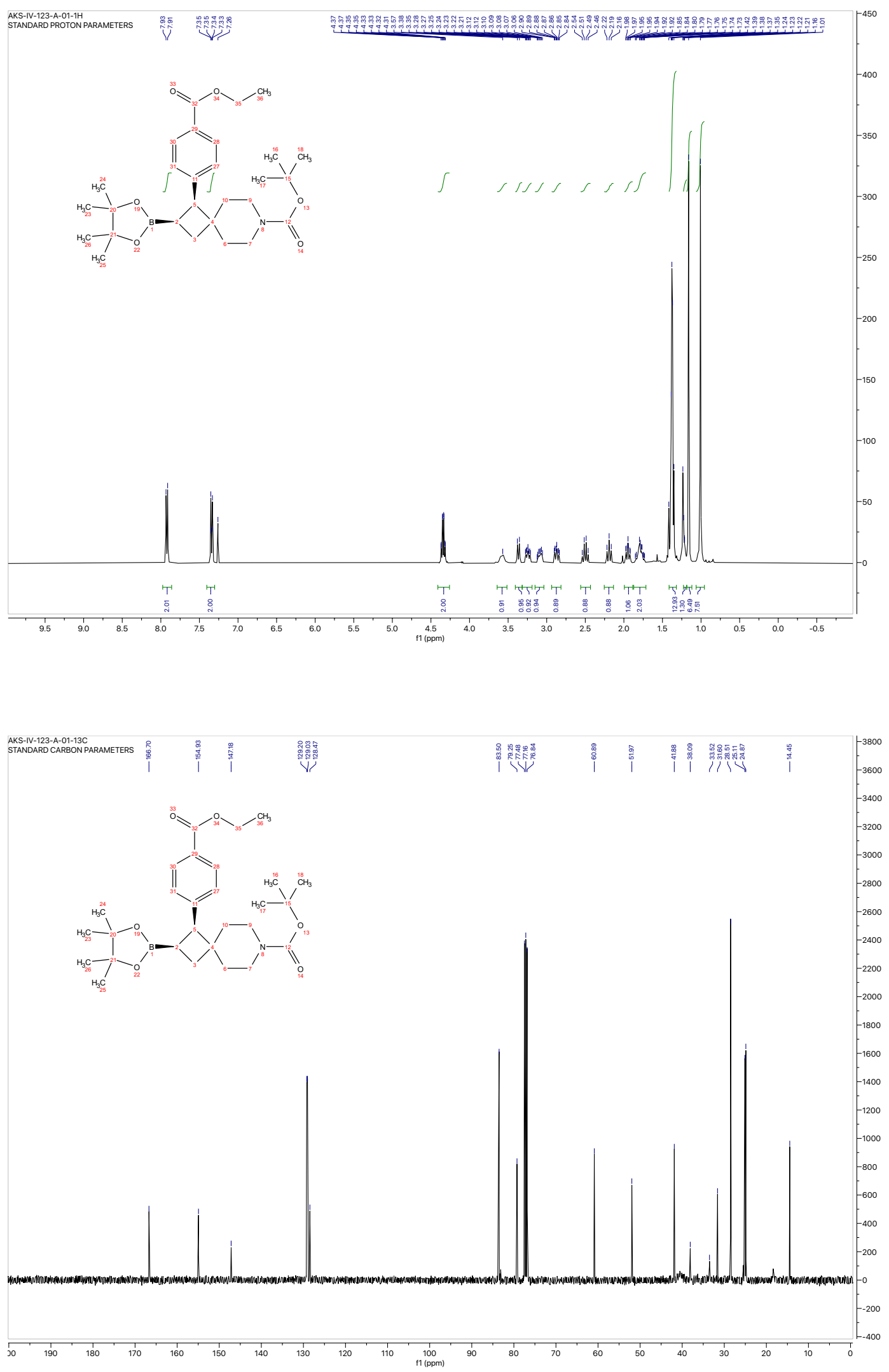

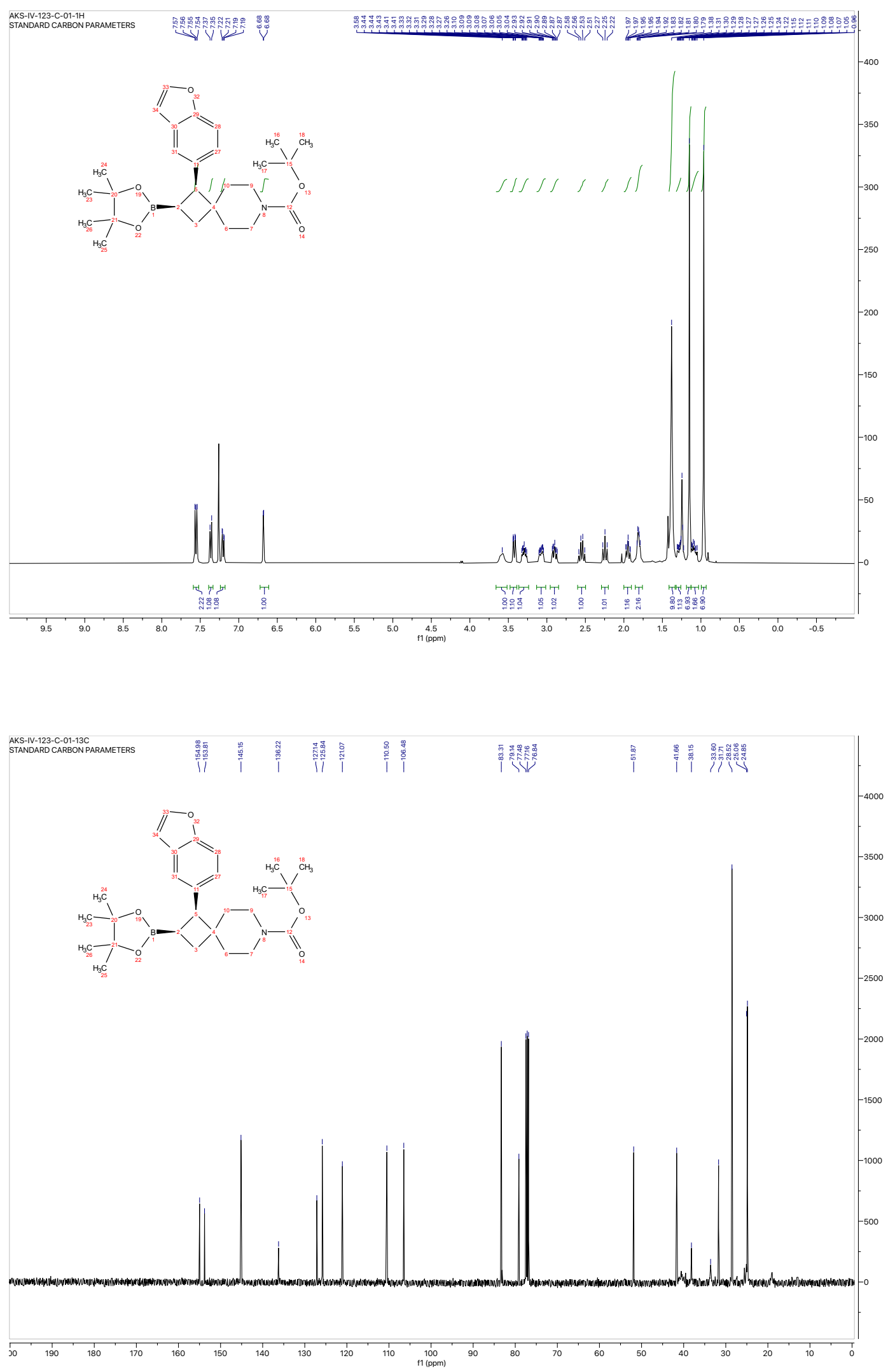

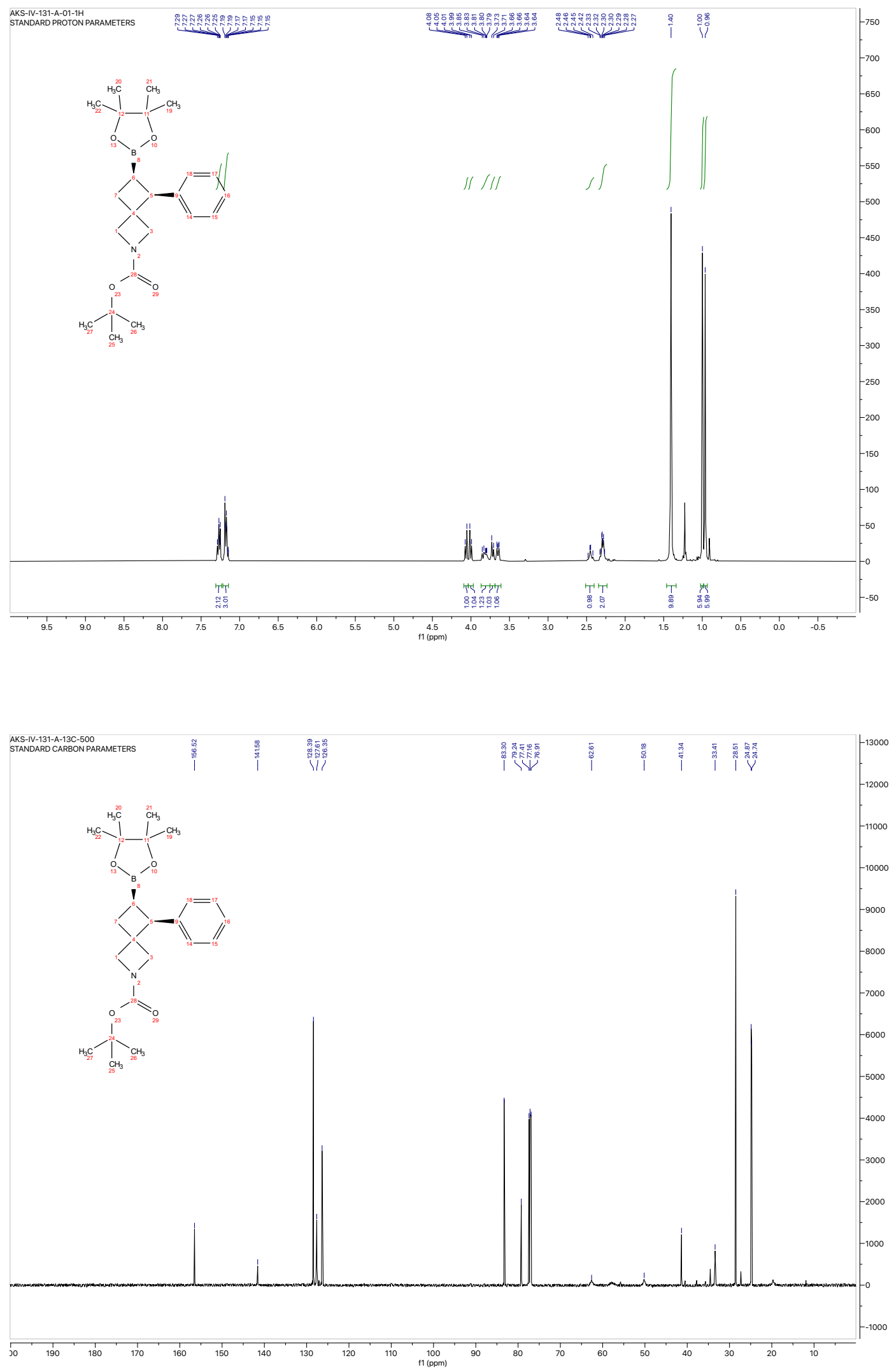

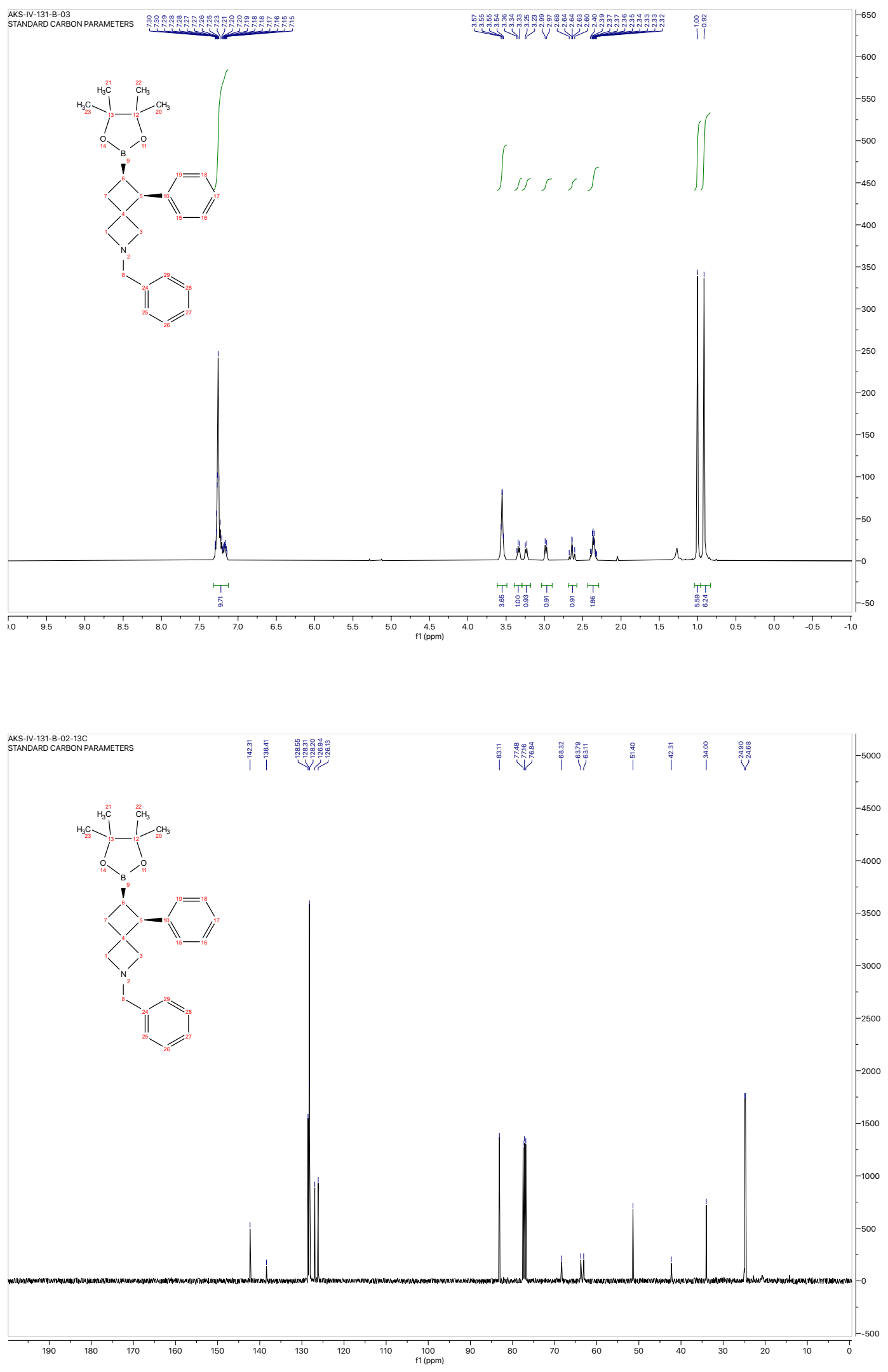

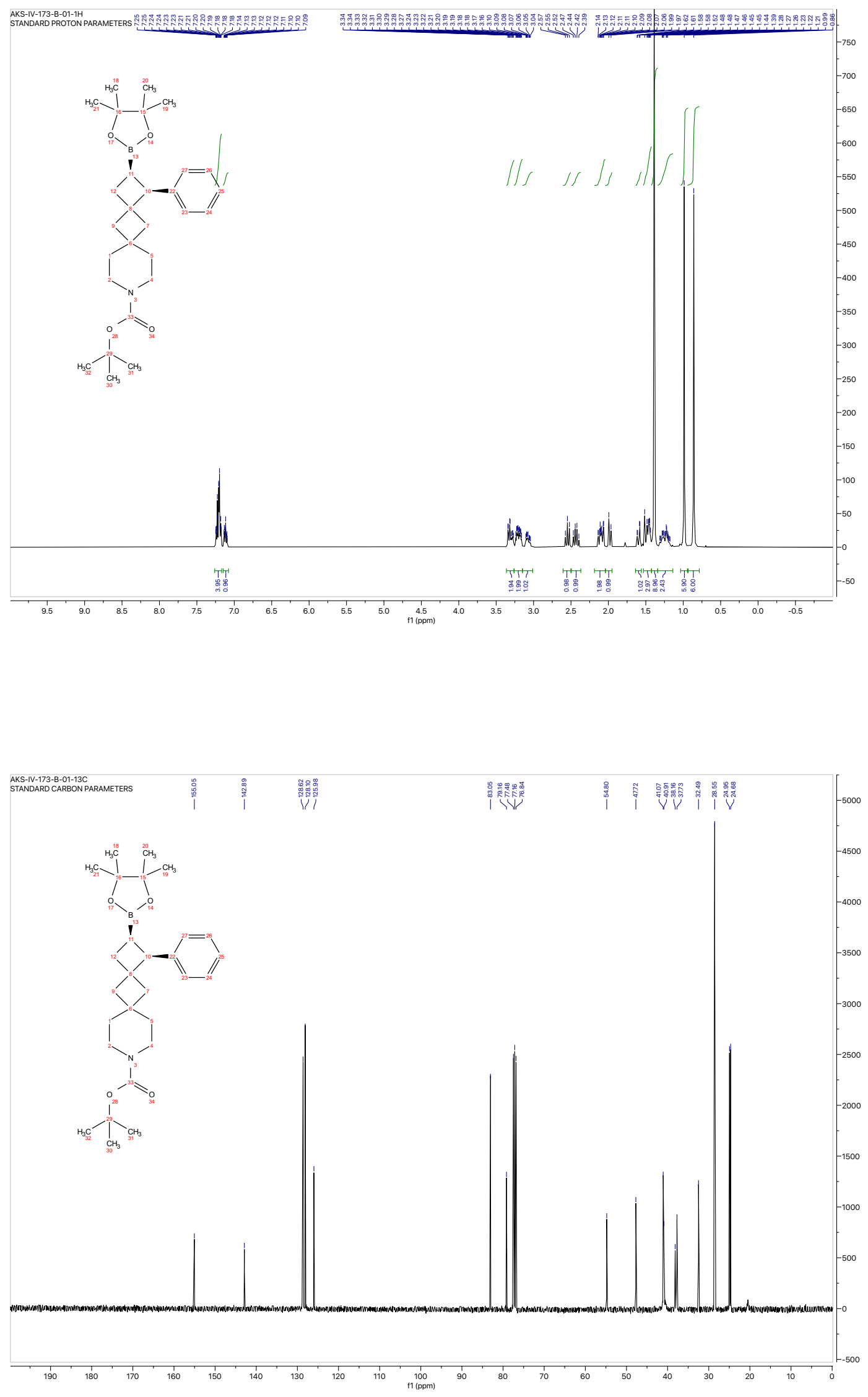

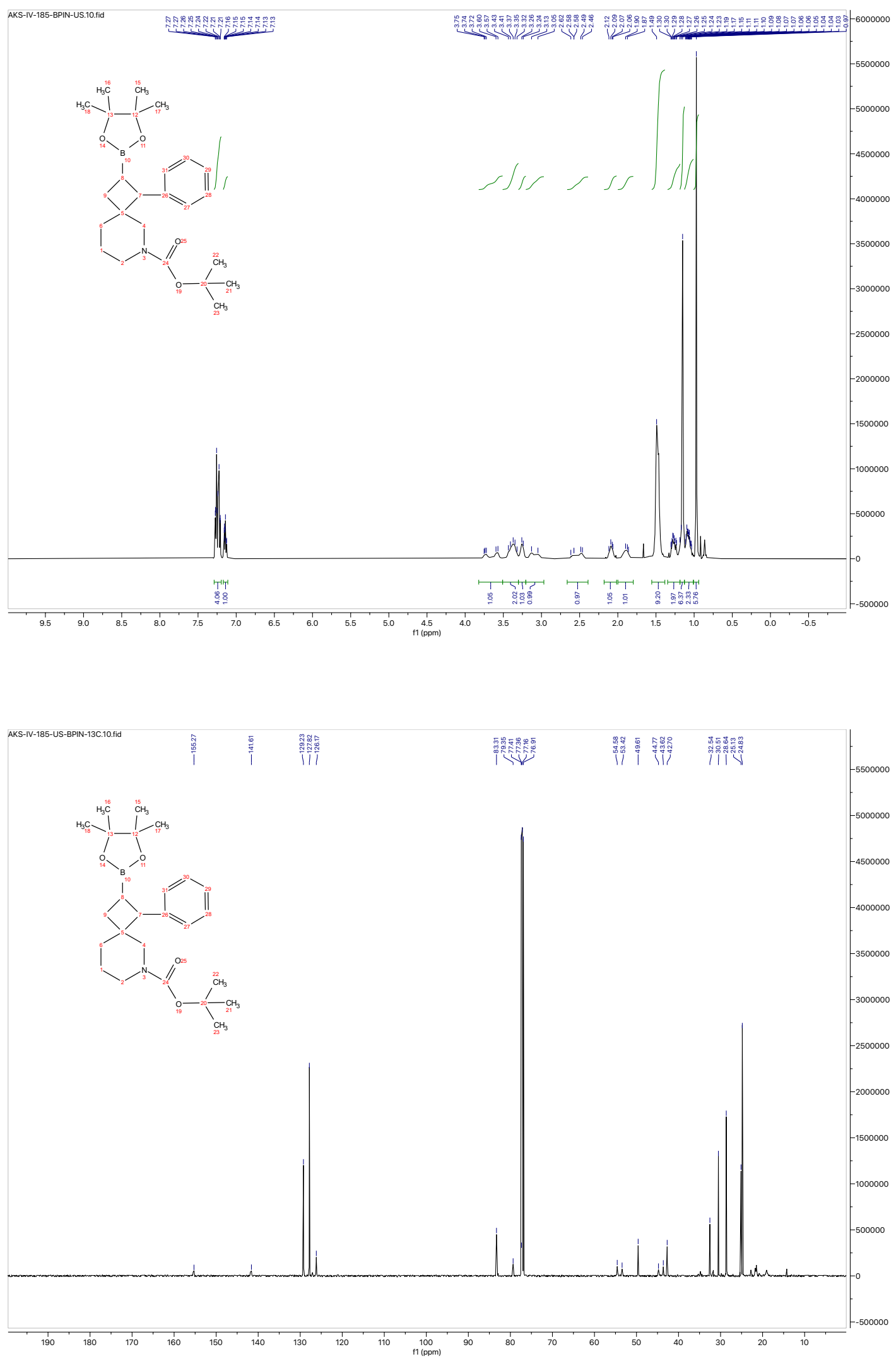

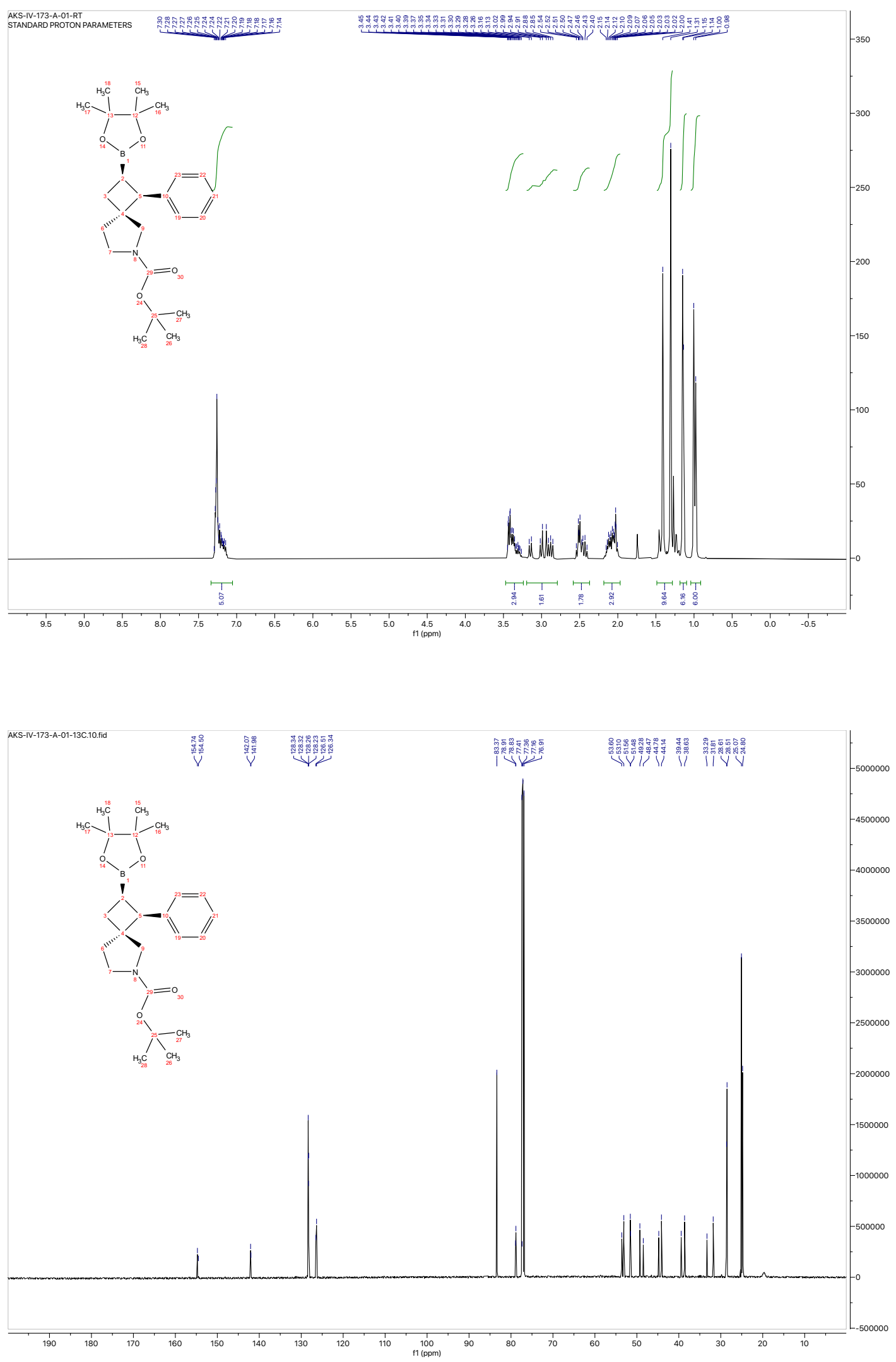

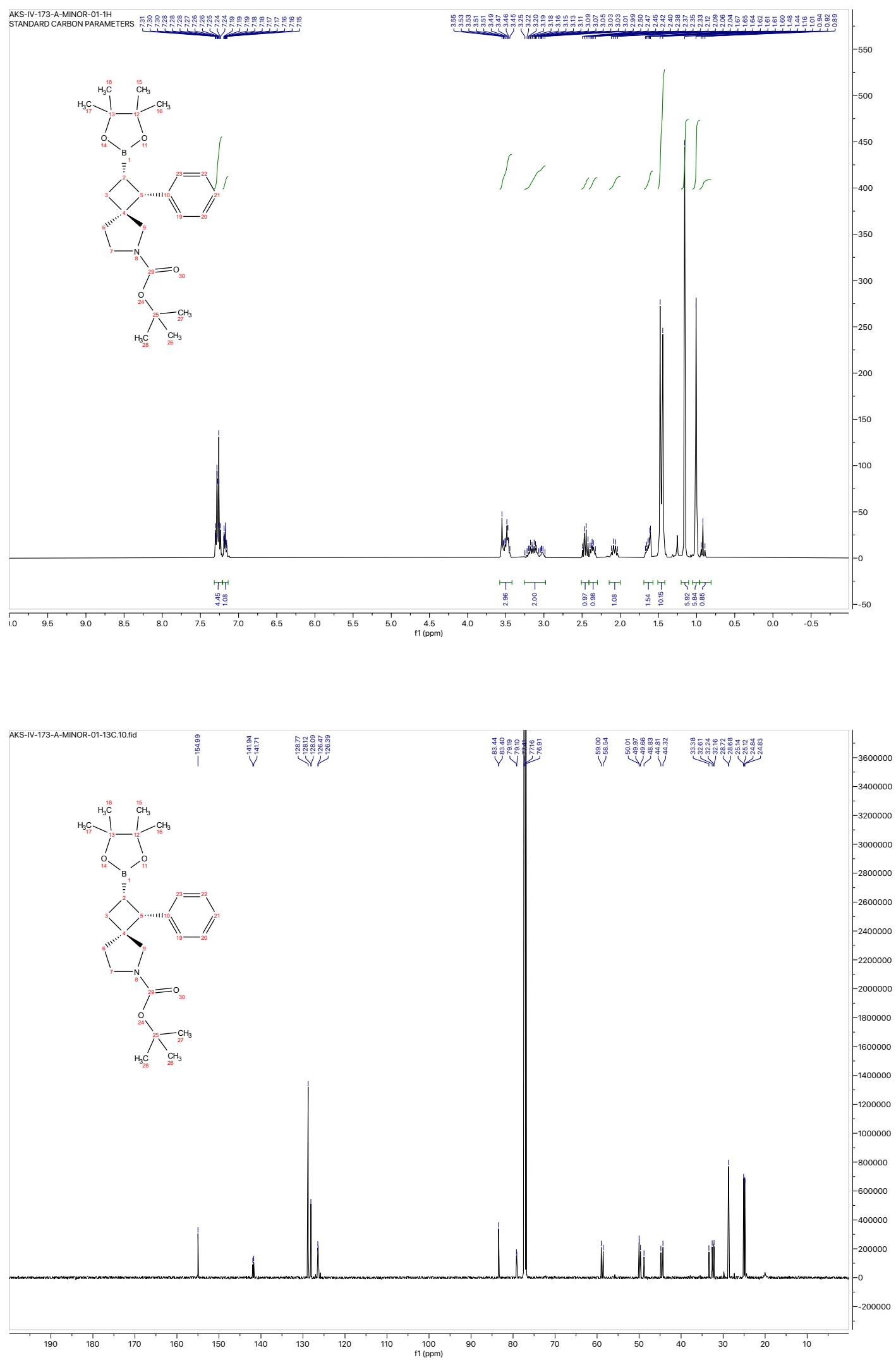

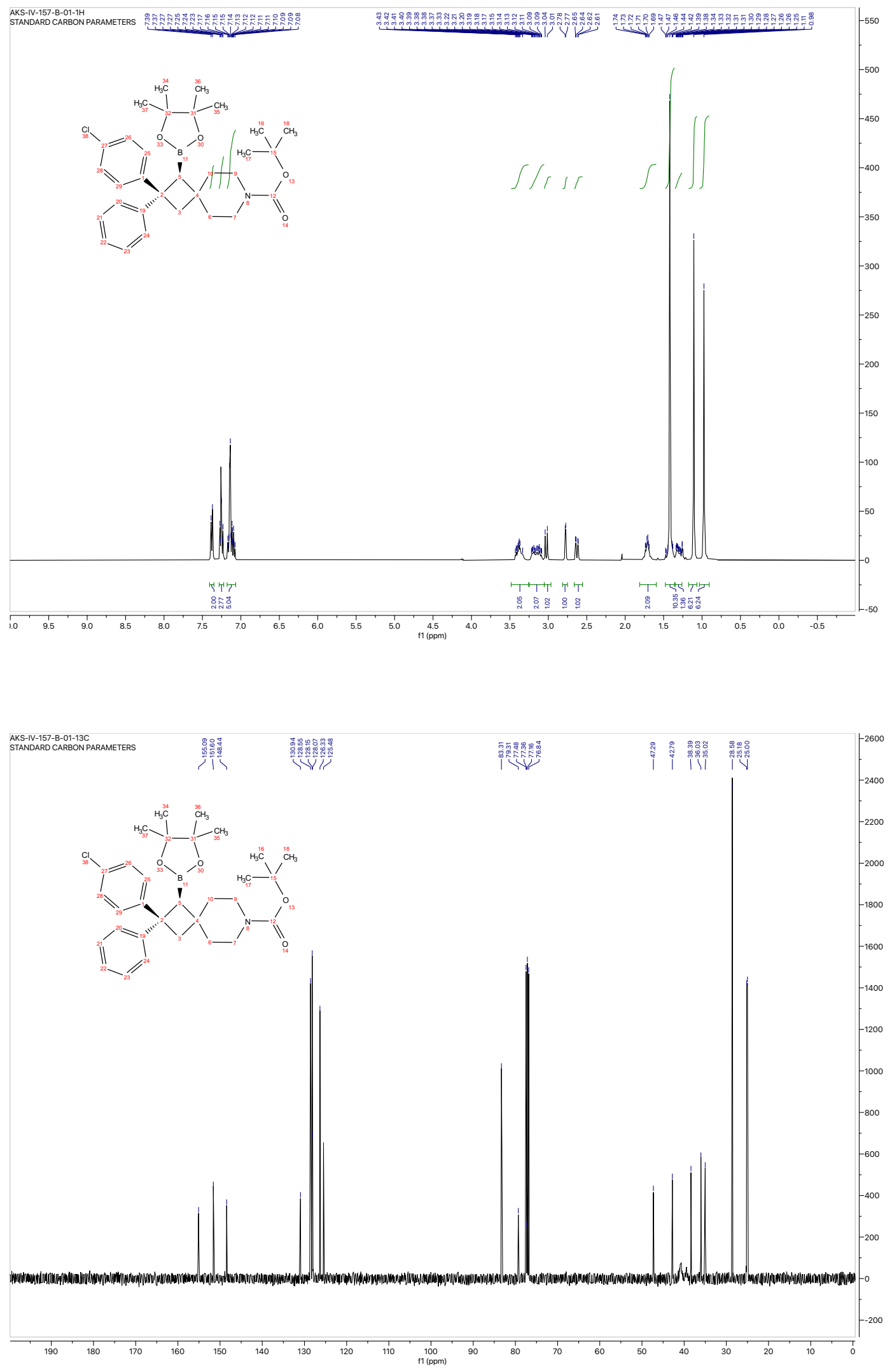

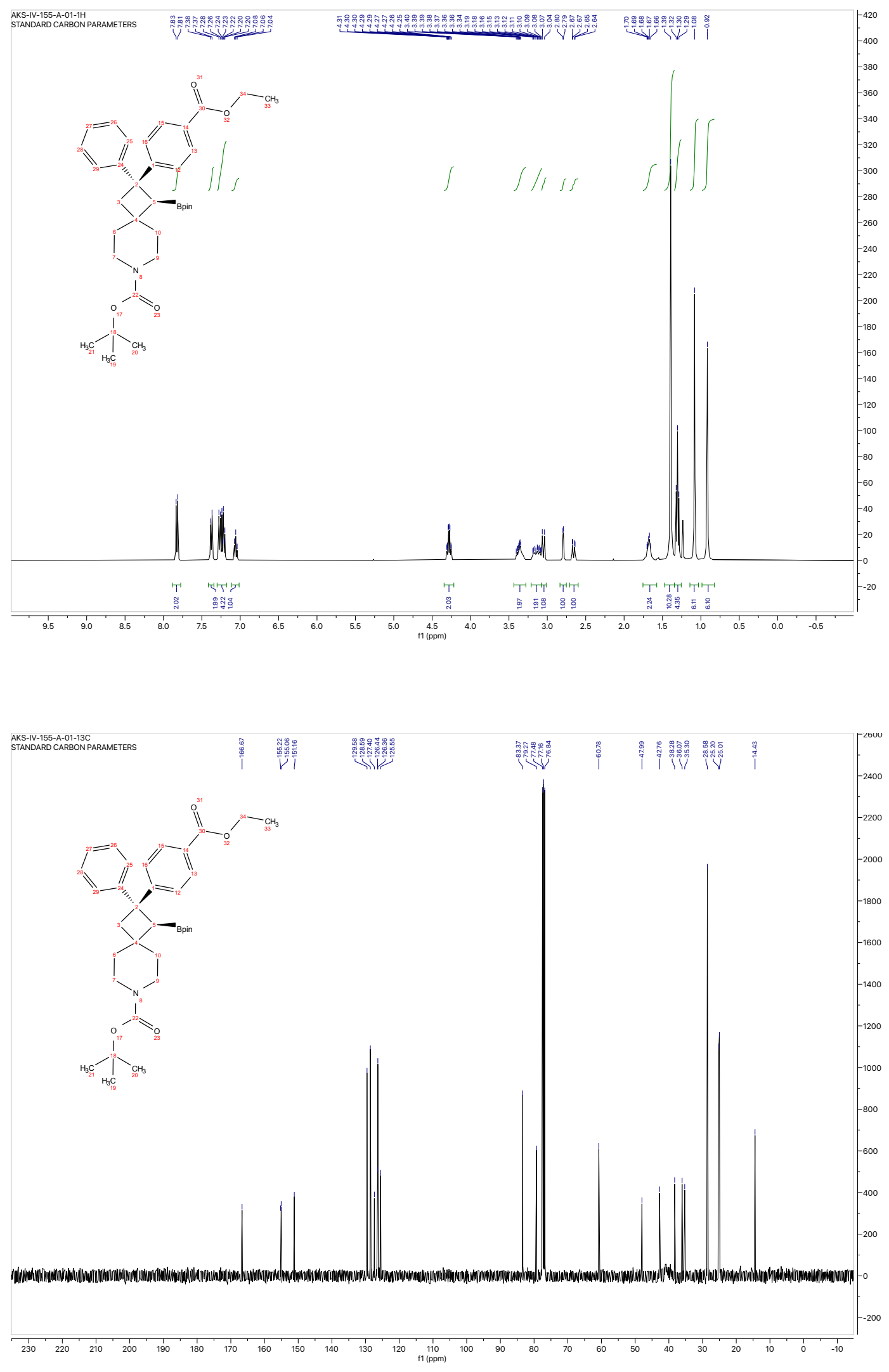

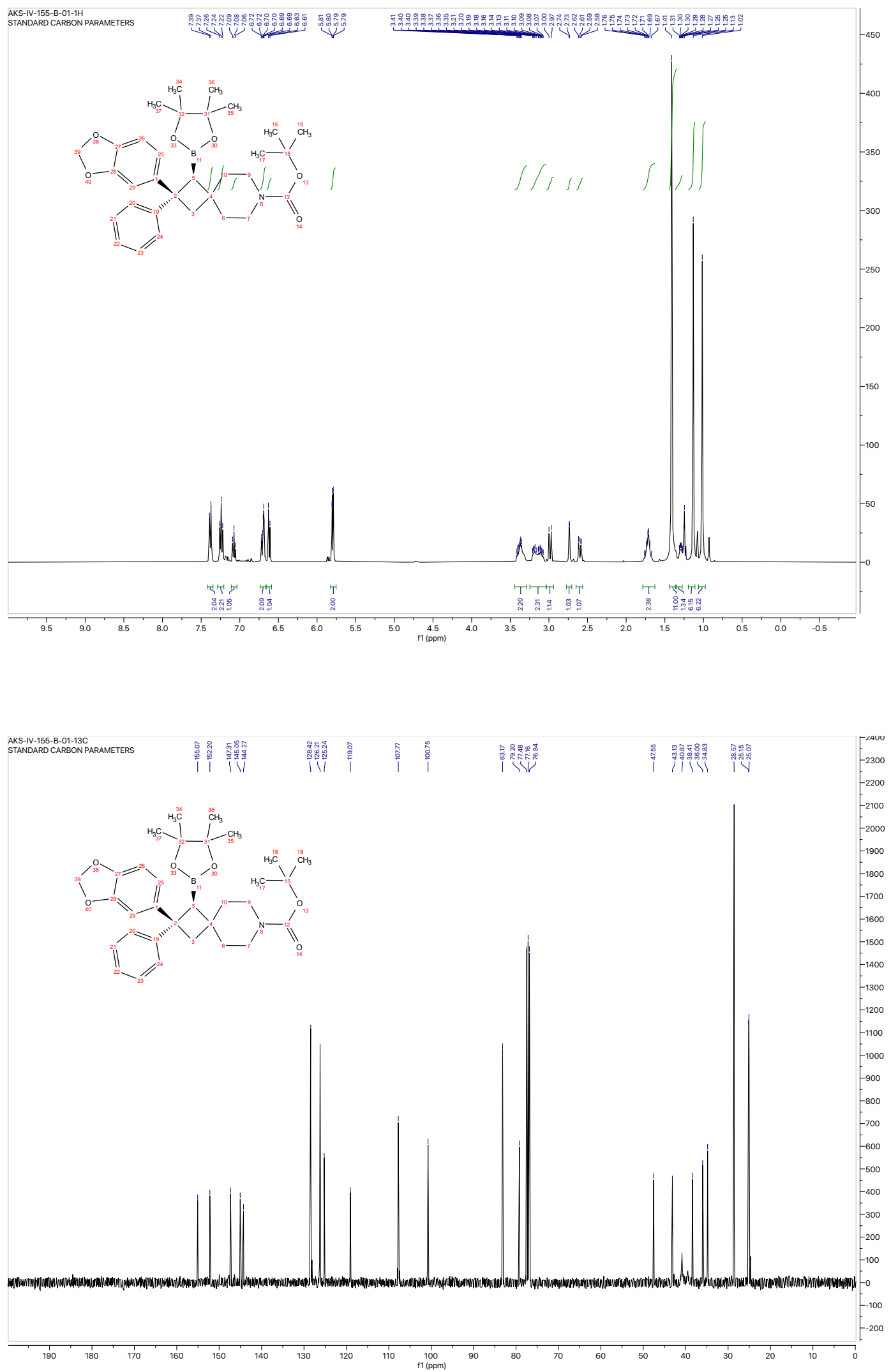

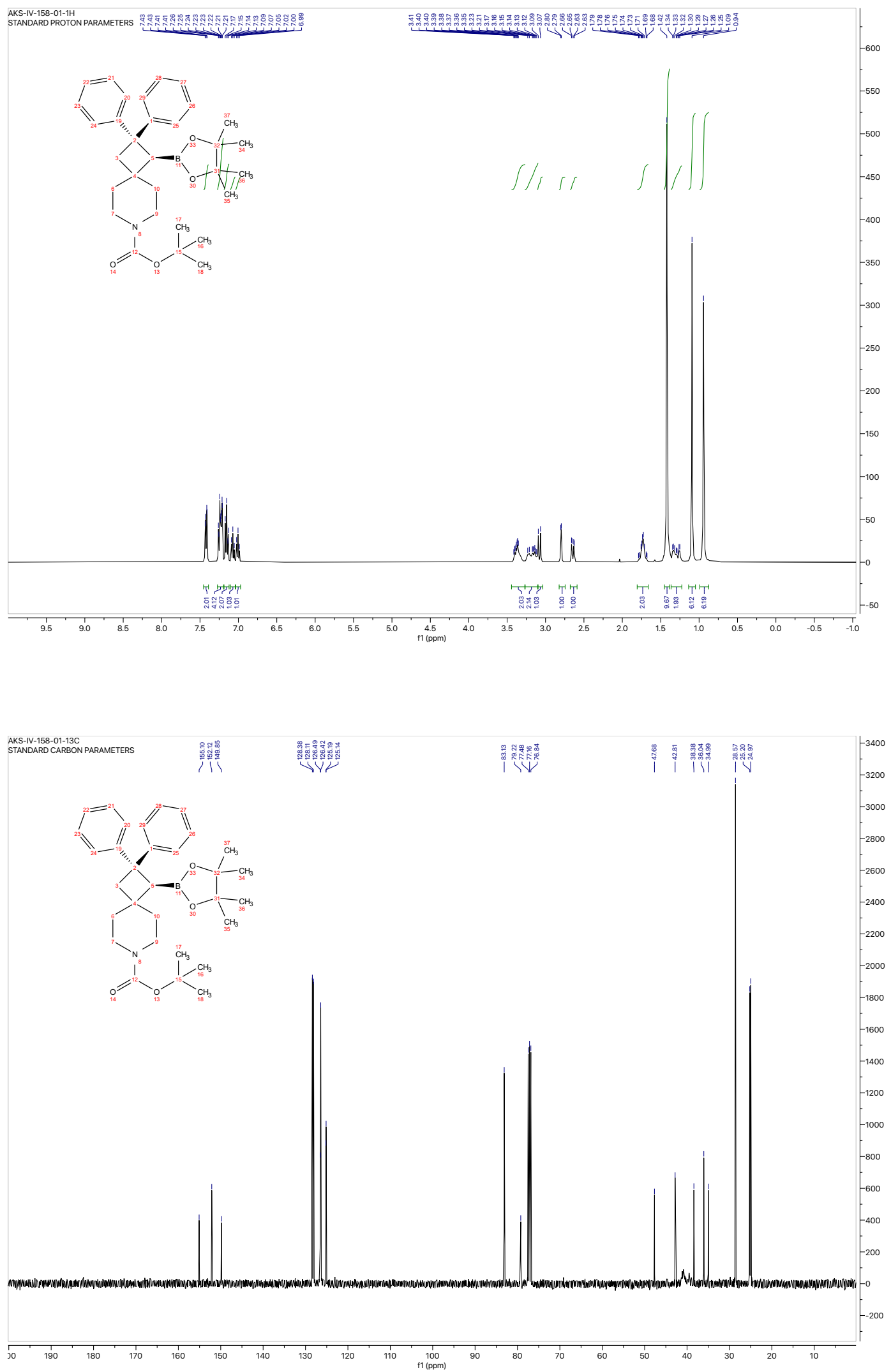

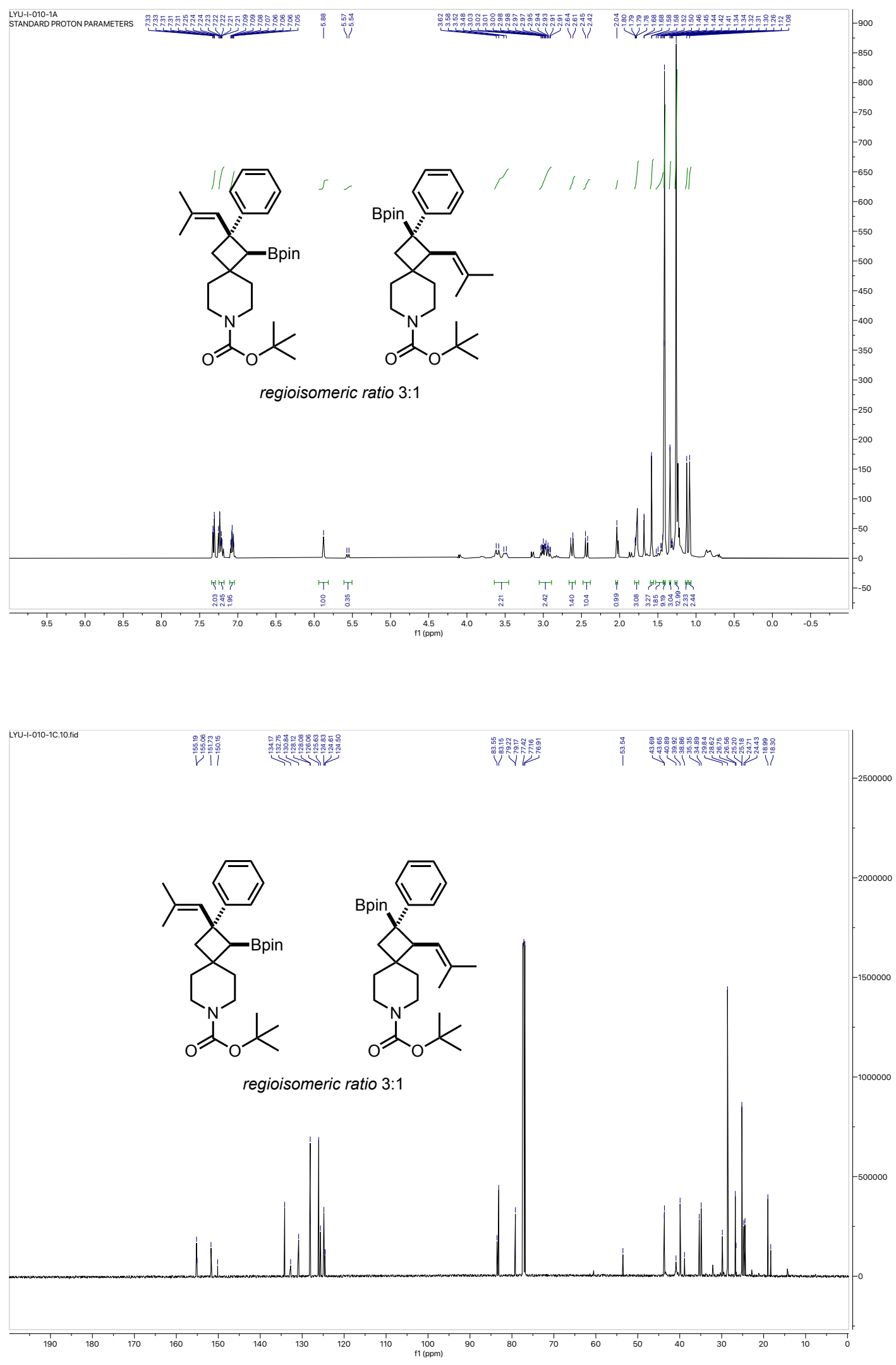

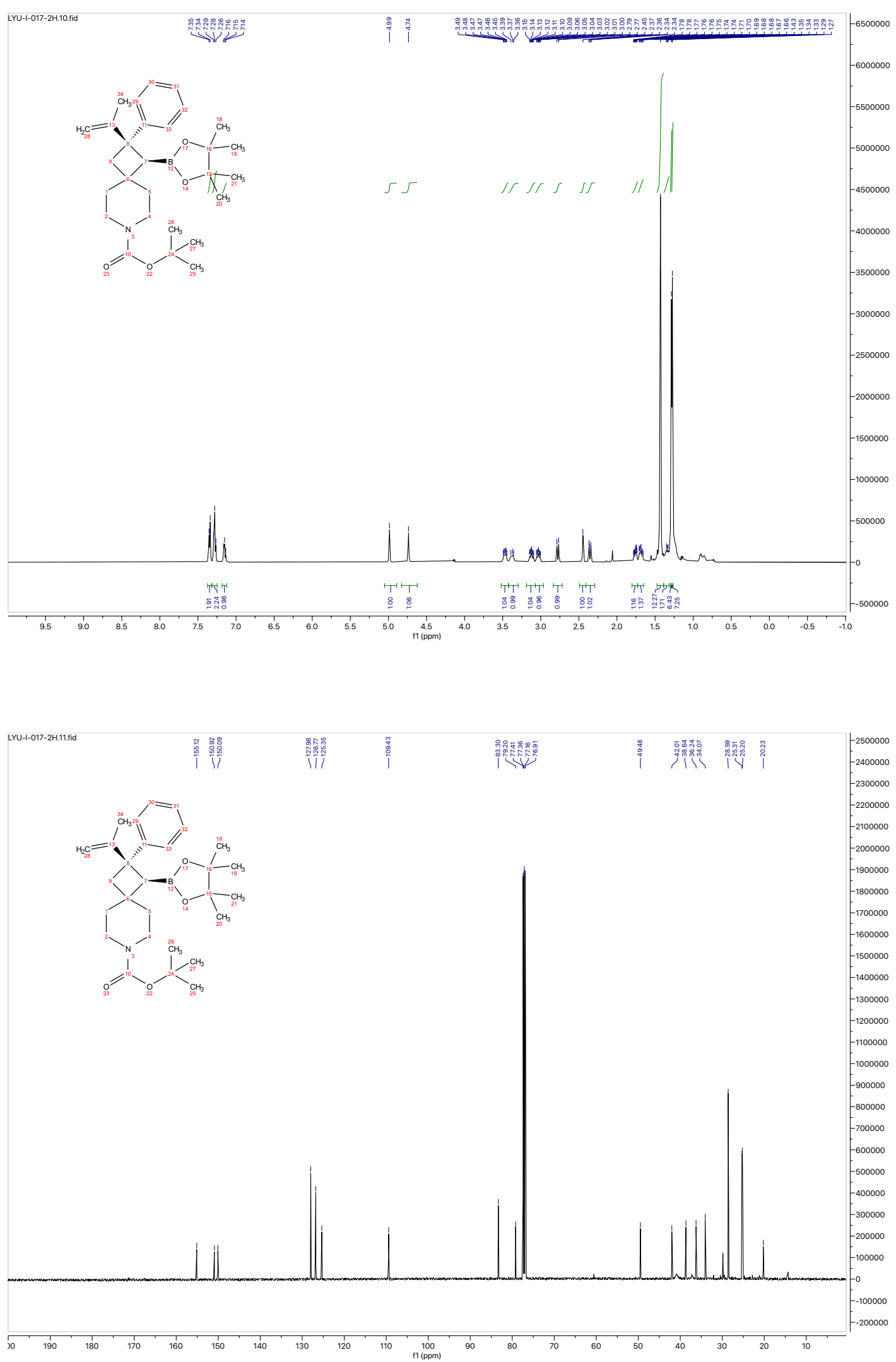

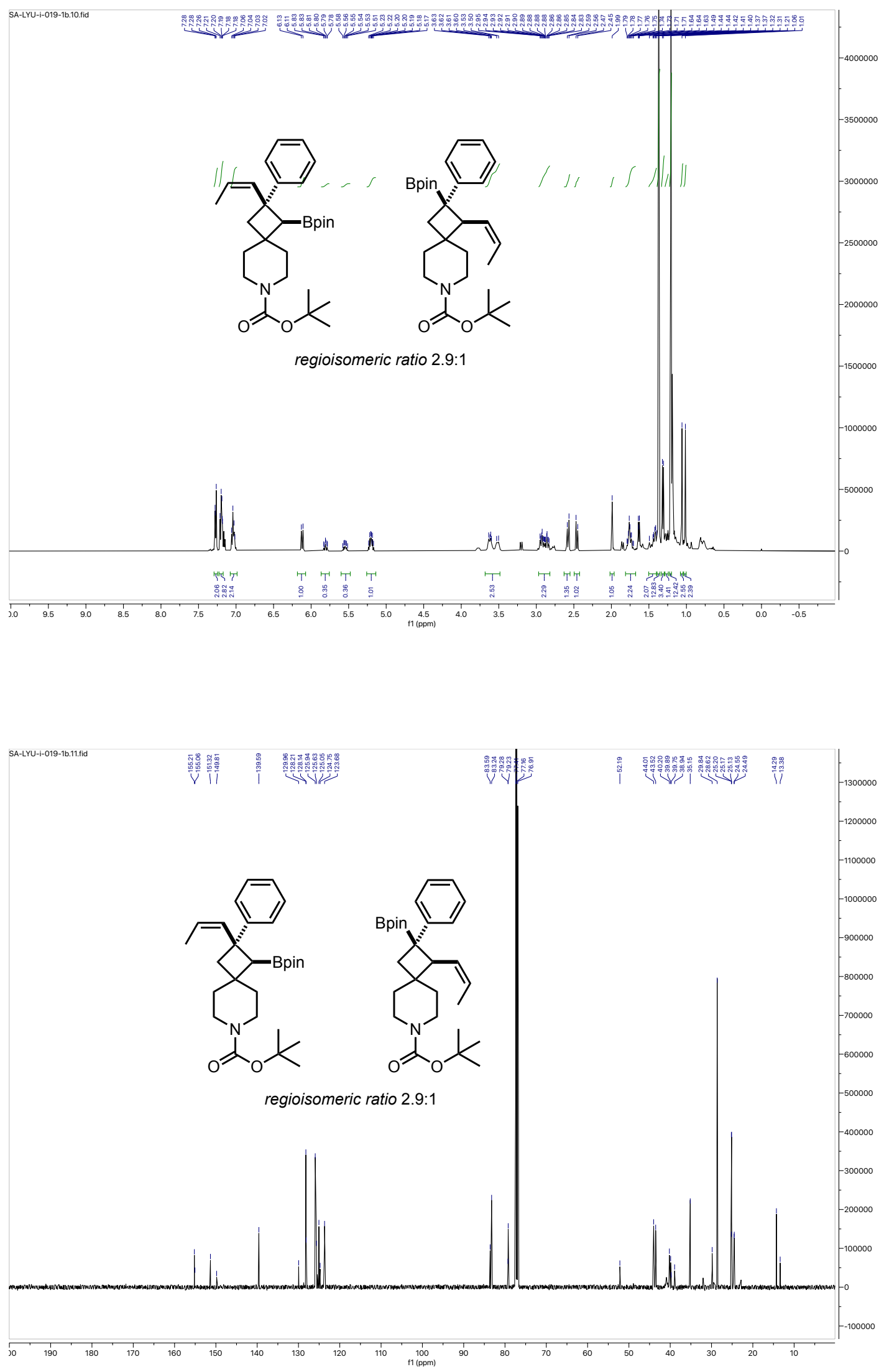

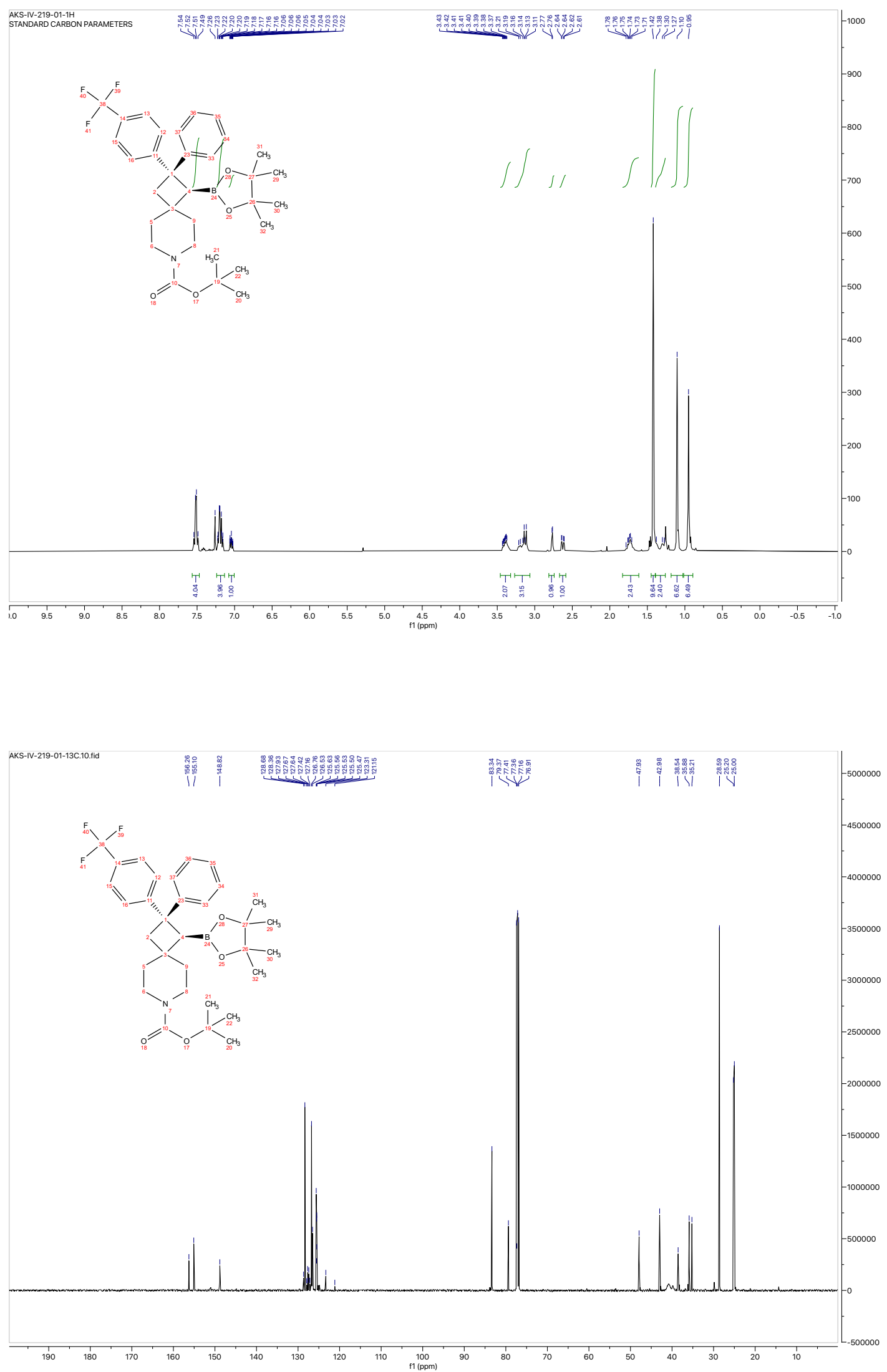


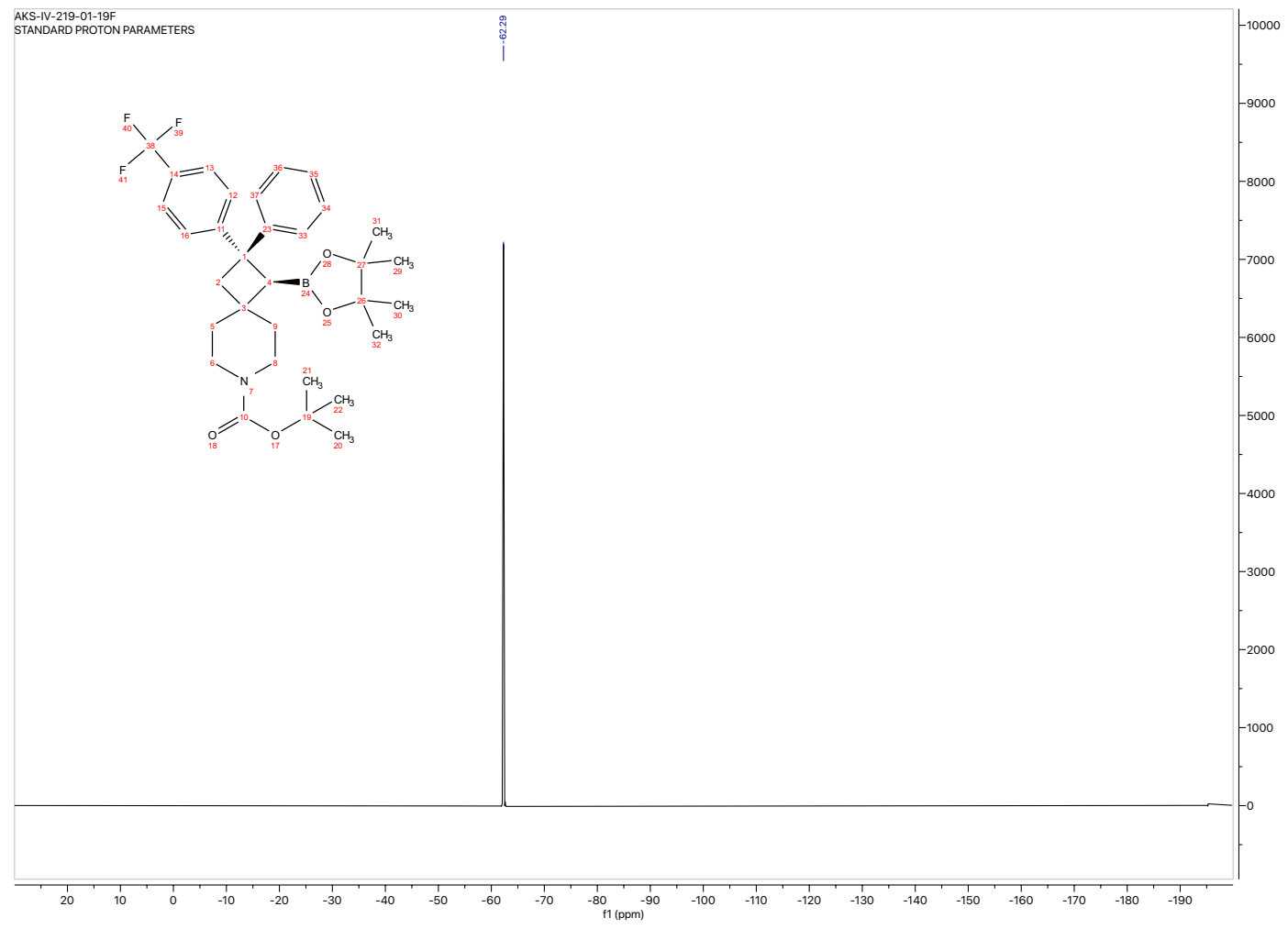



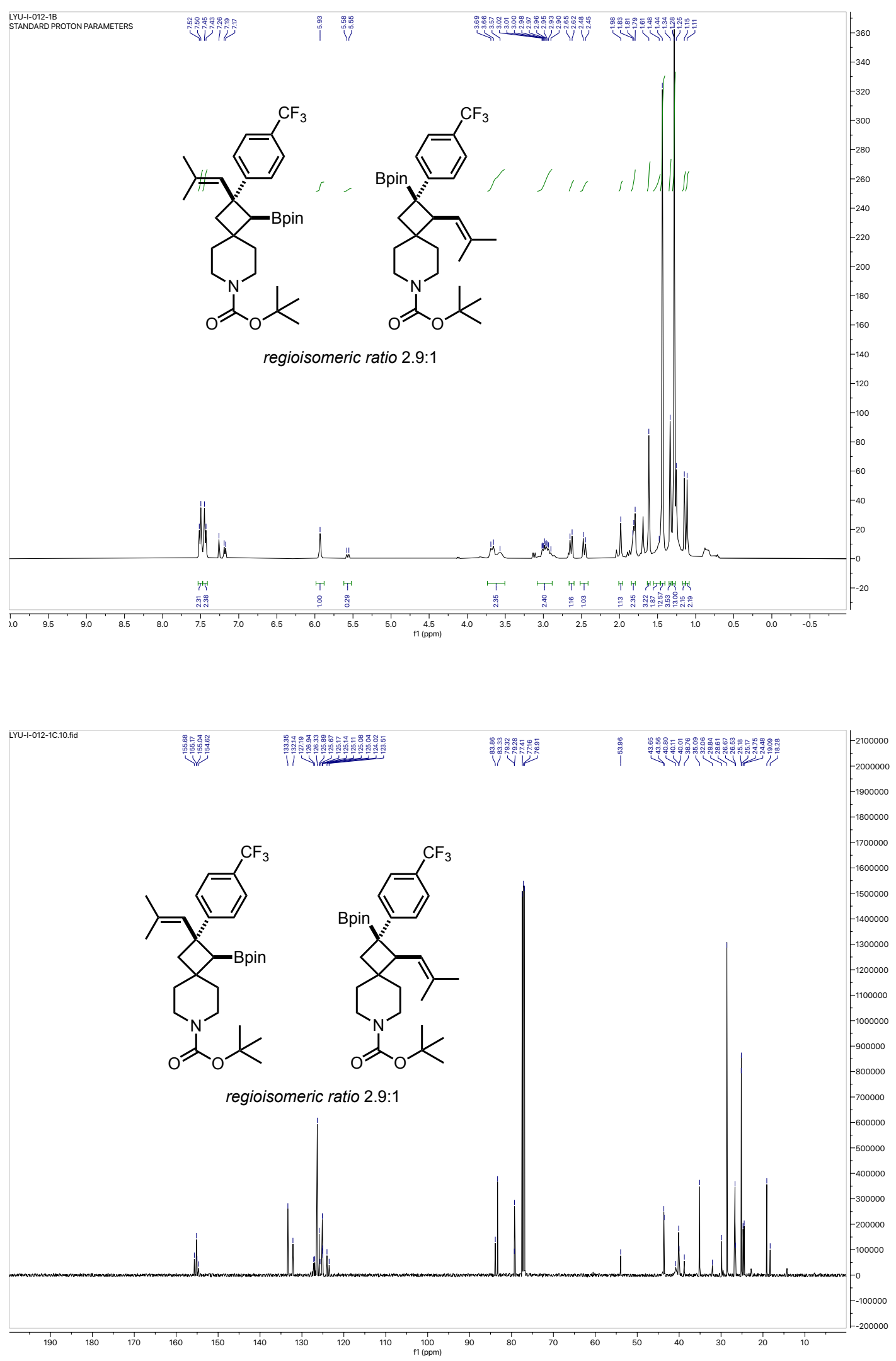
Arylboration of Spirocyclic Cyclobutenes

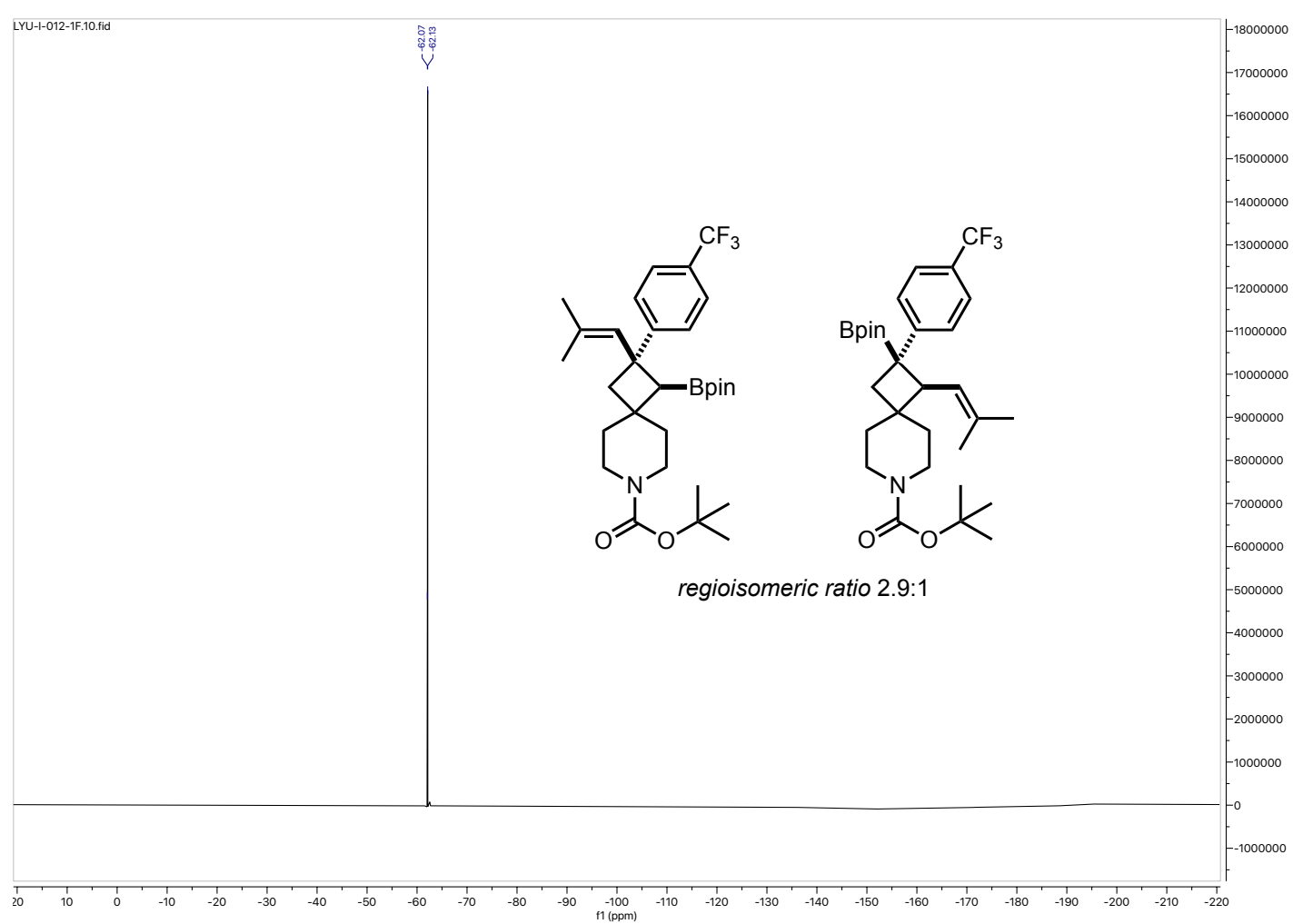



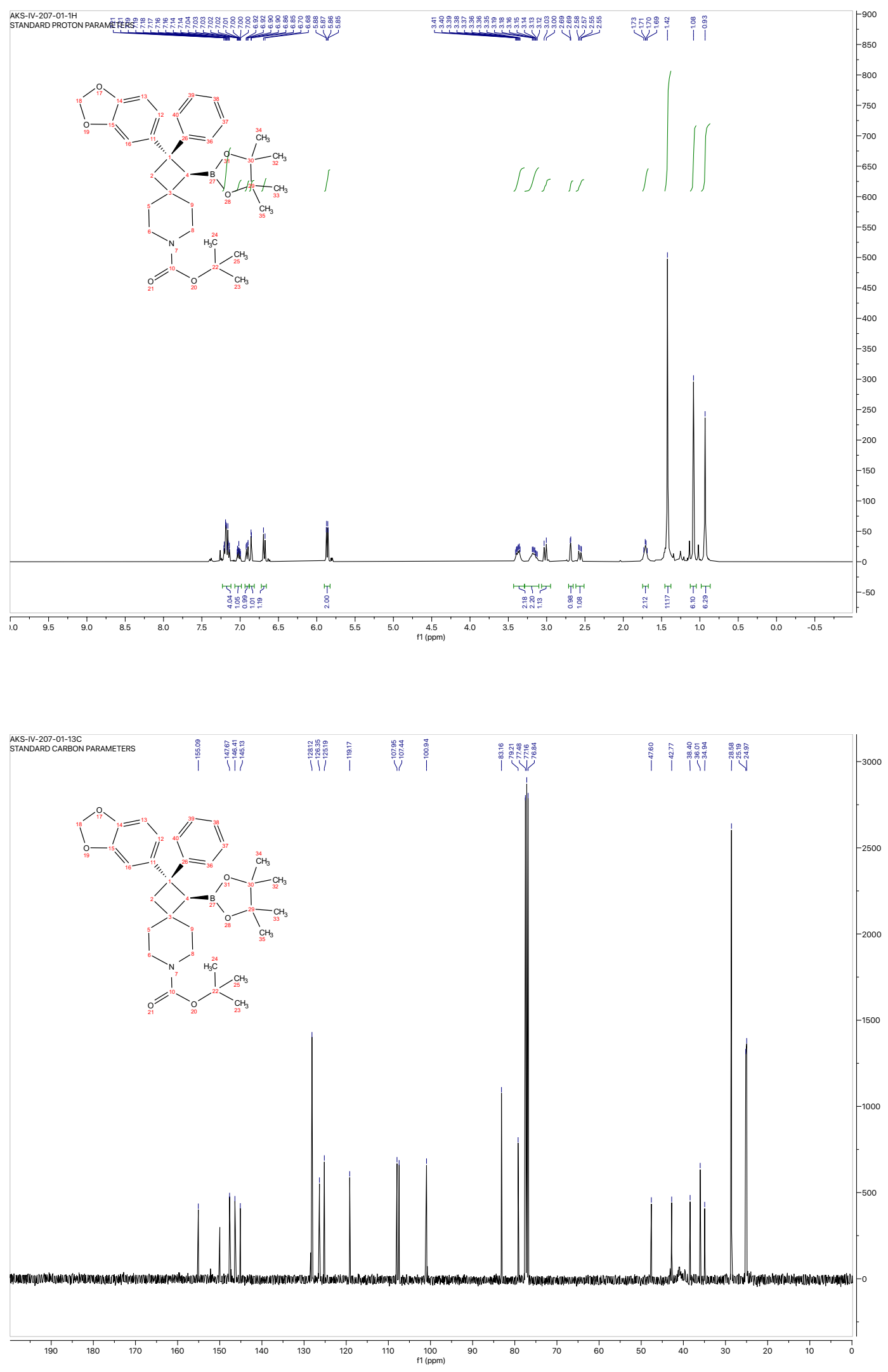

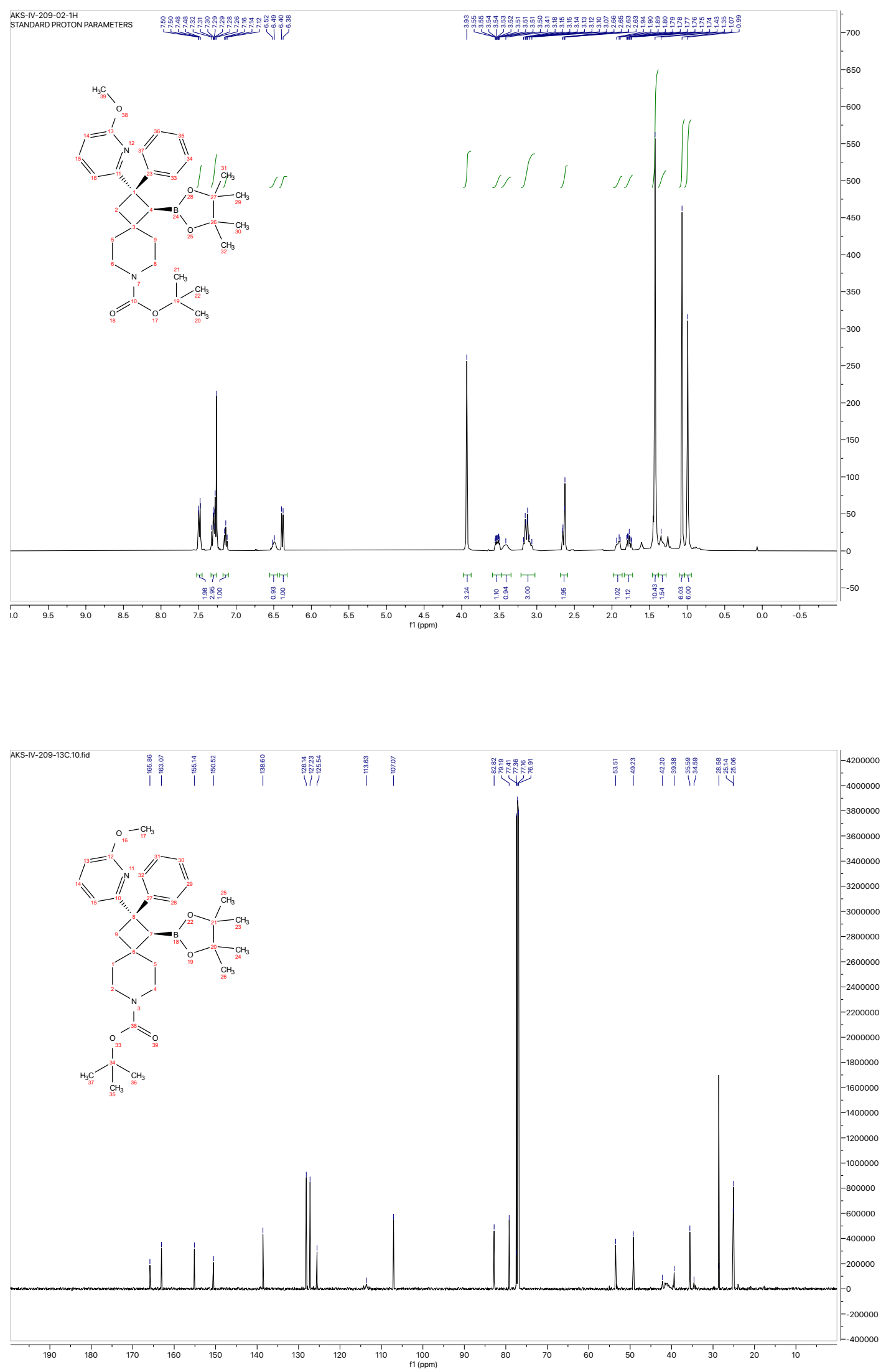

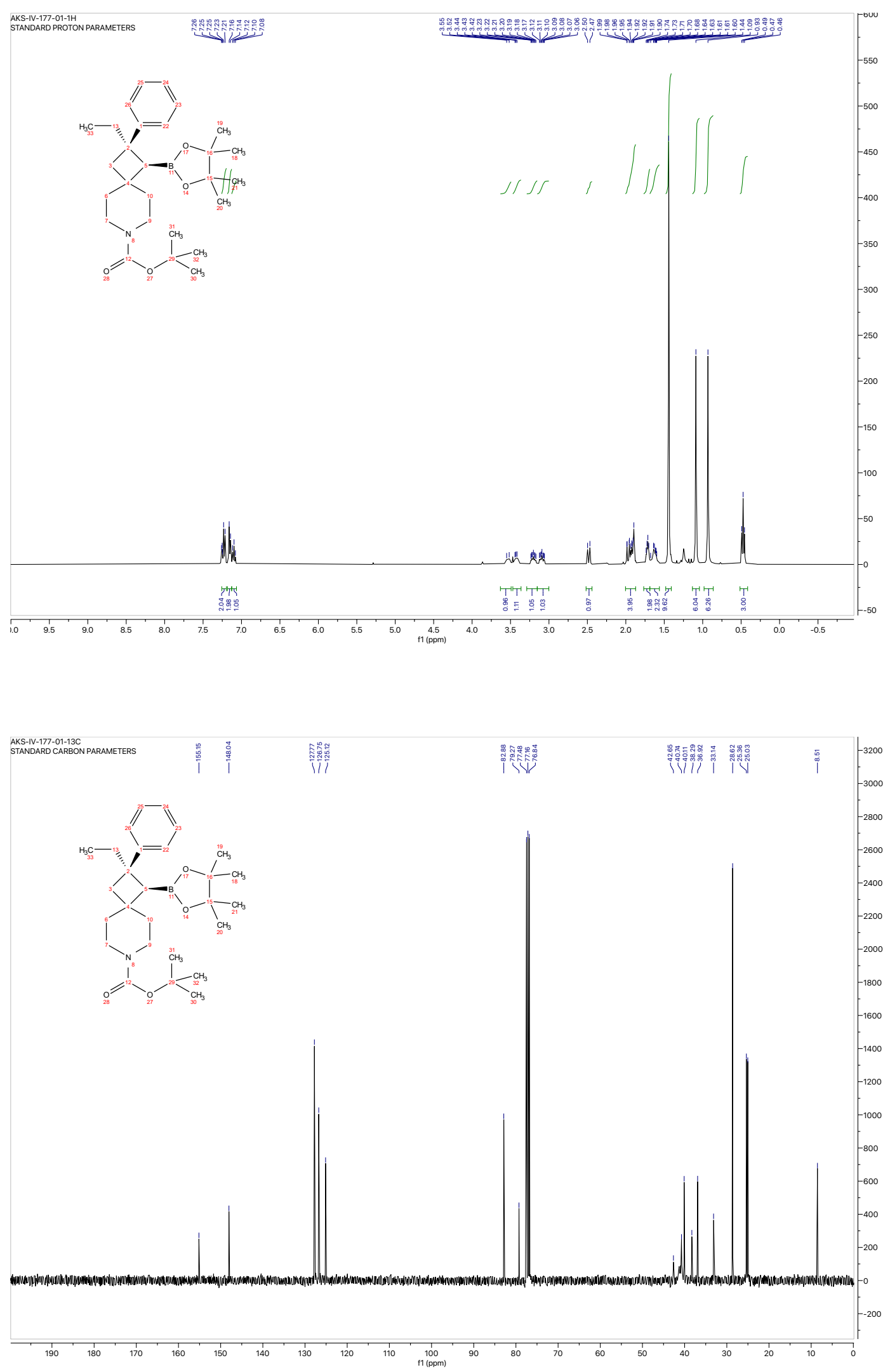

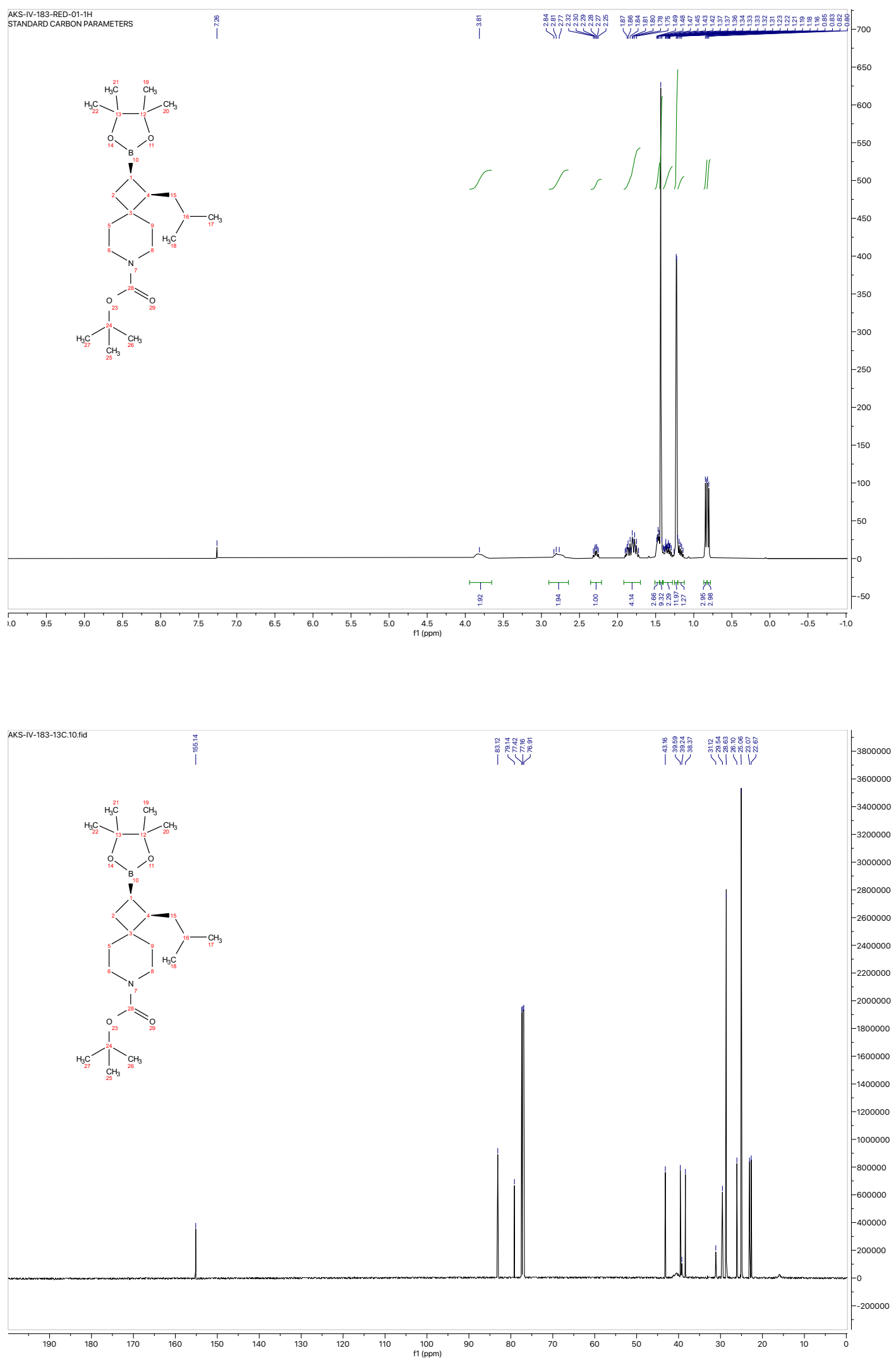

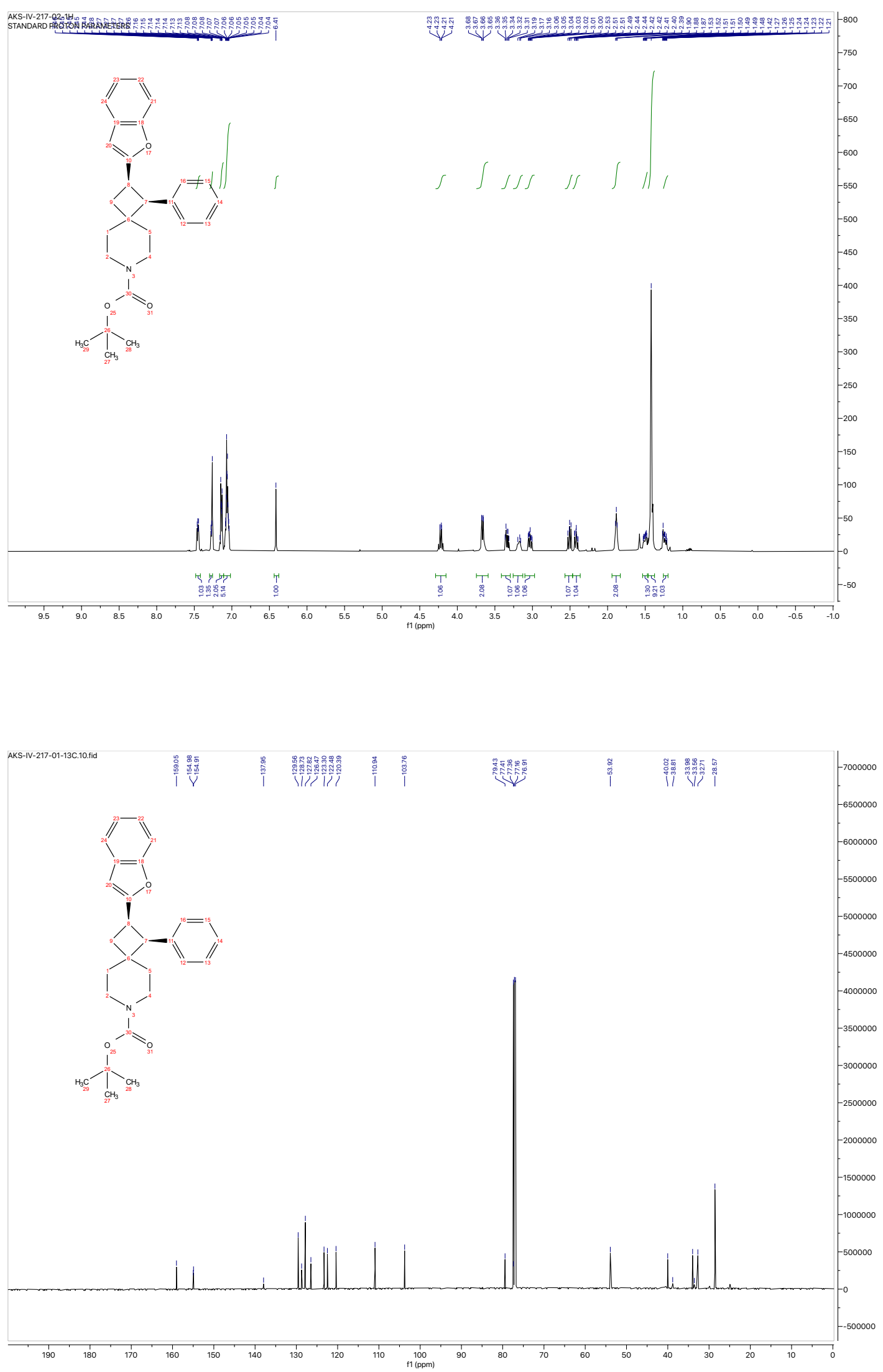

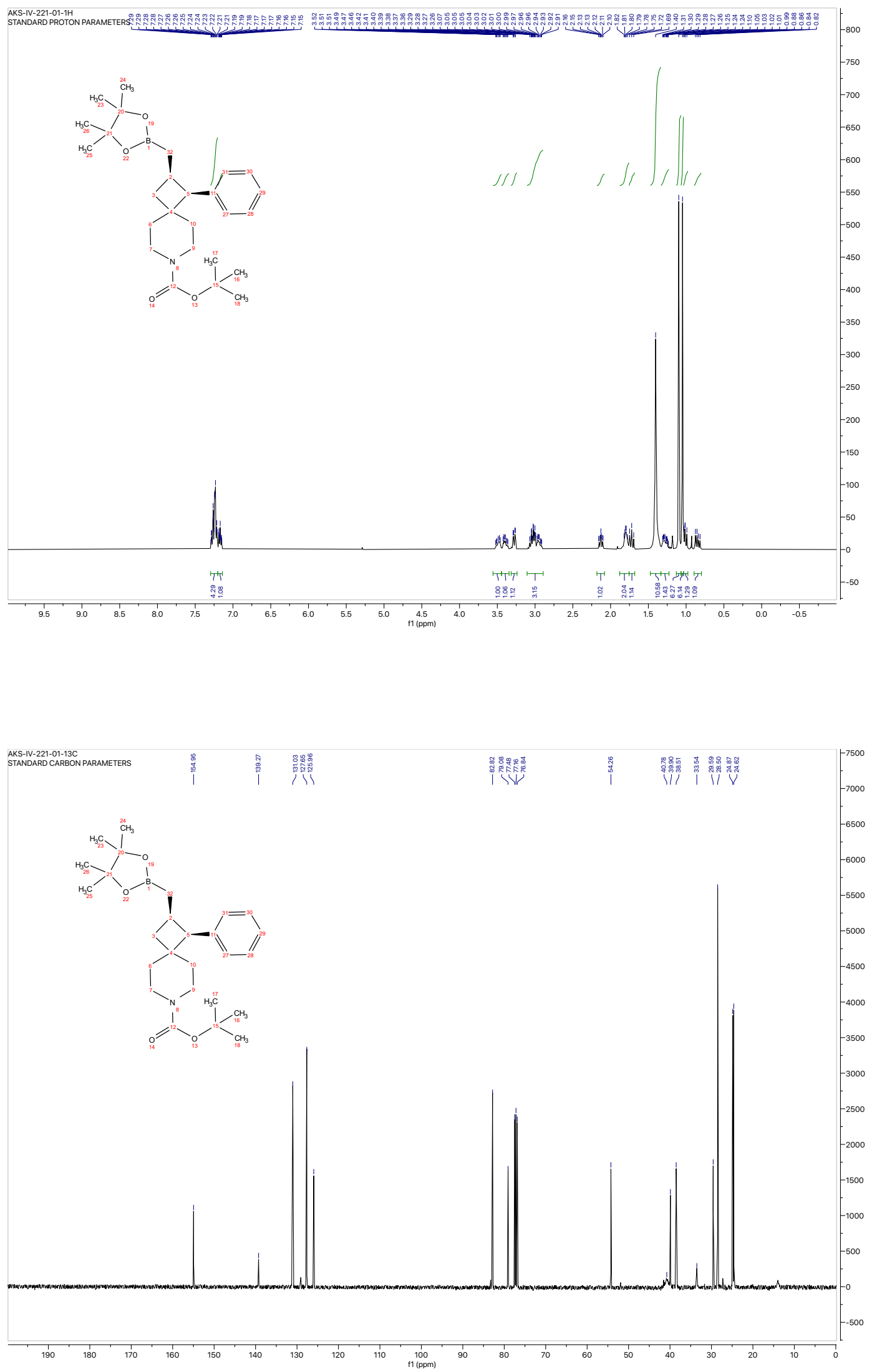

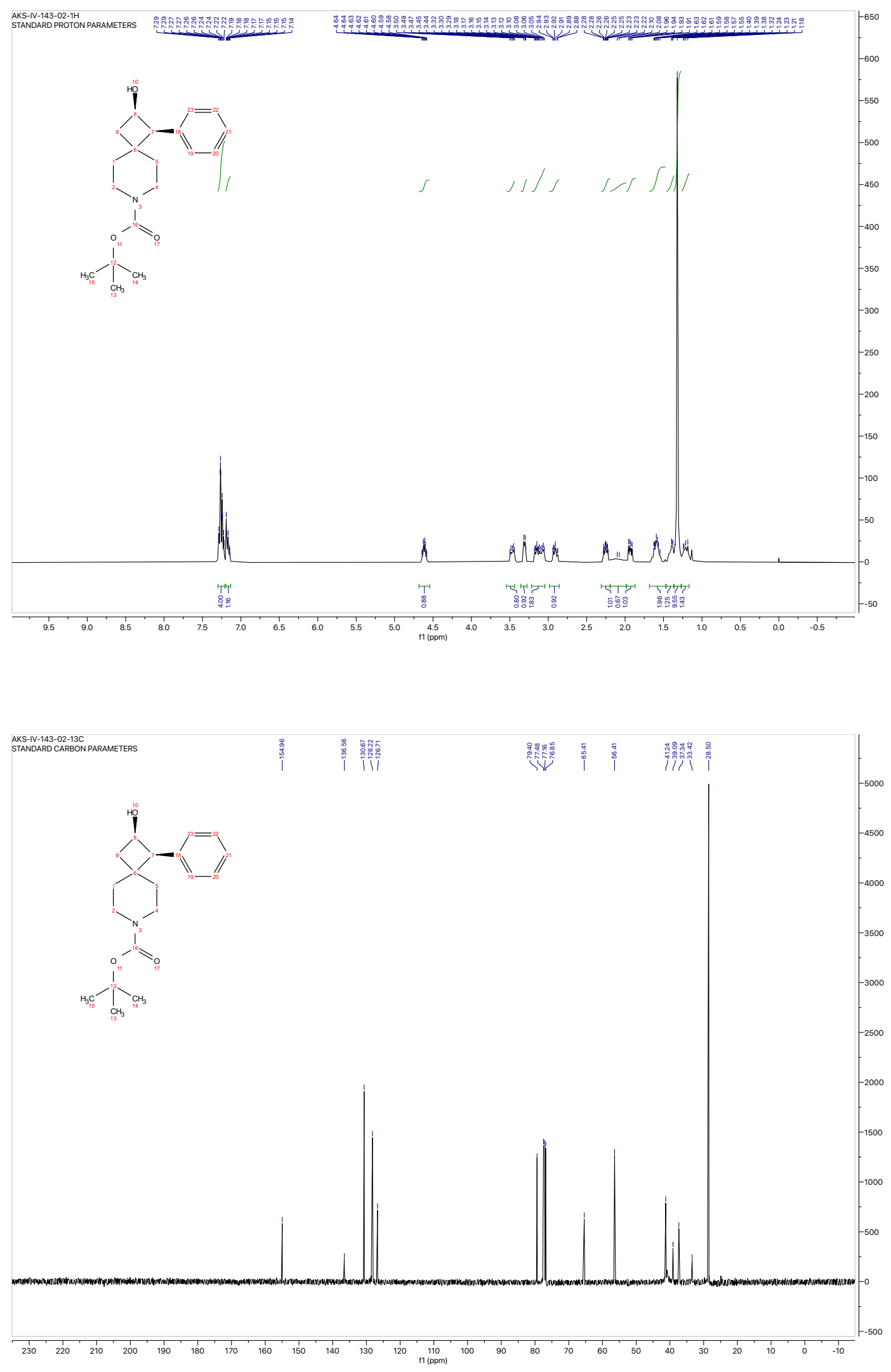

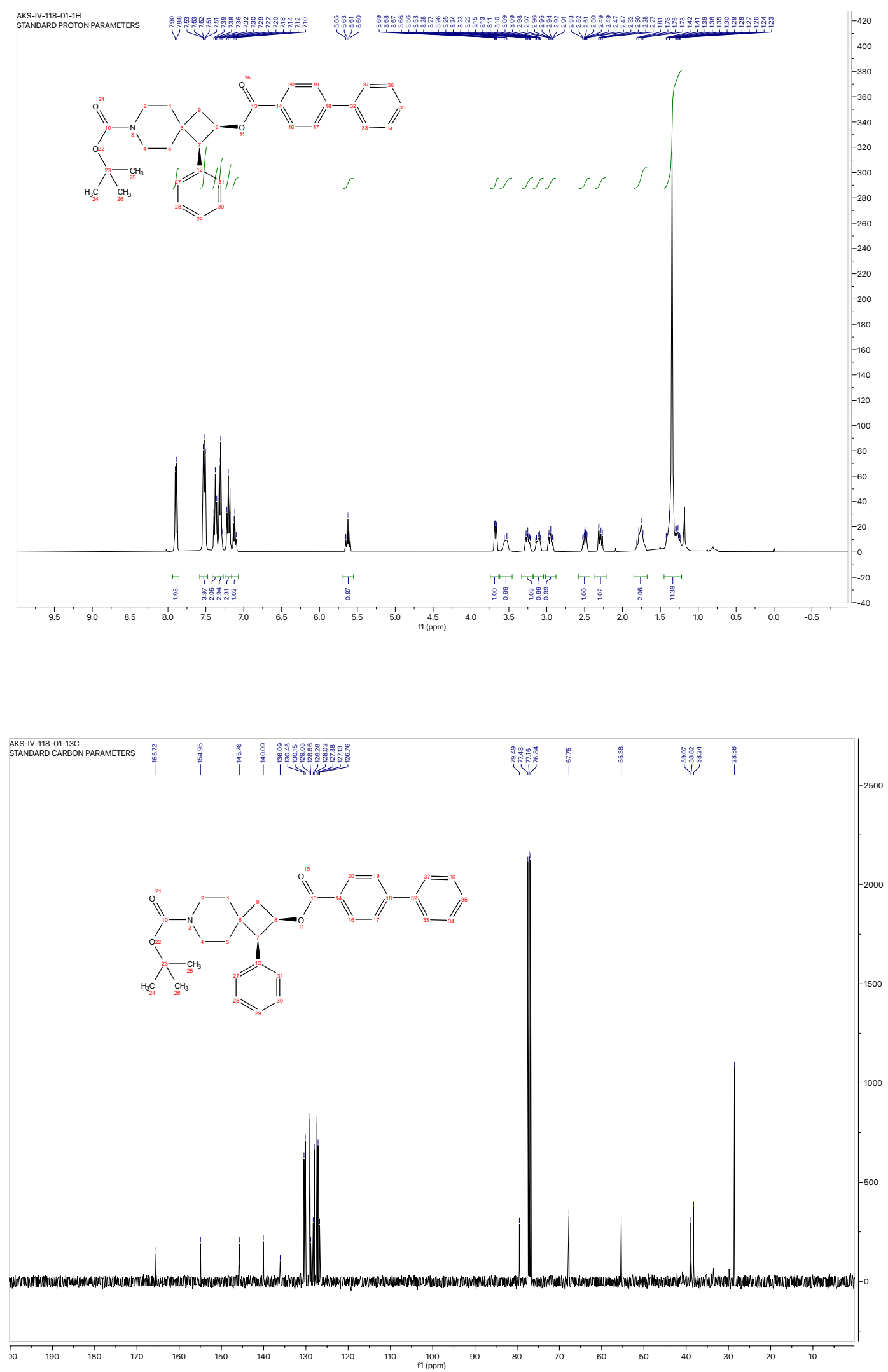

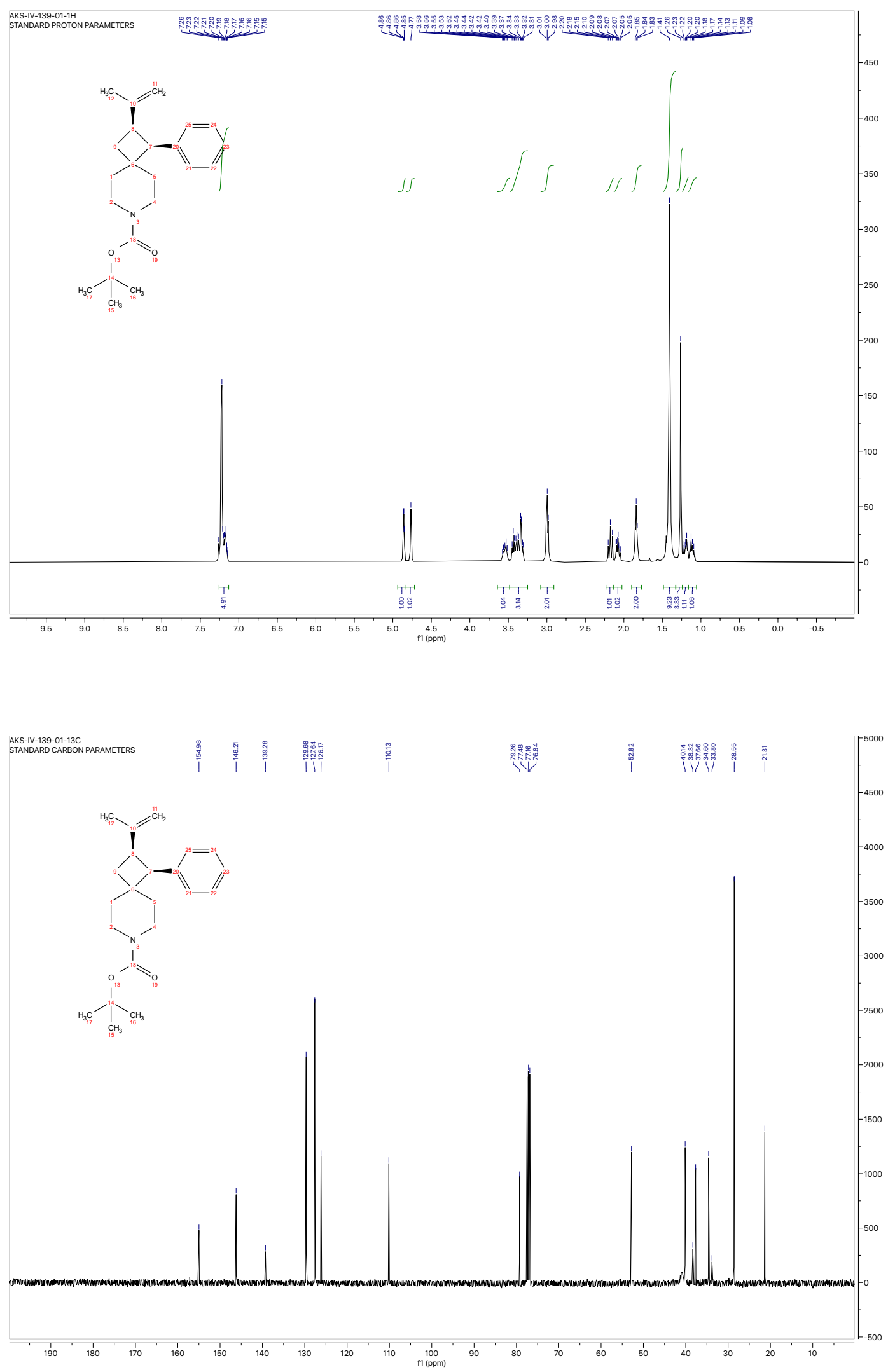

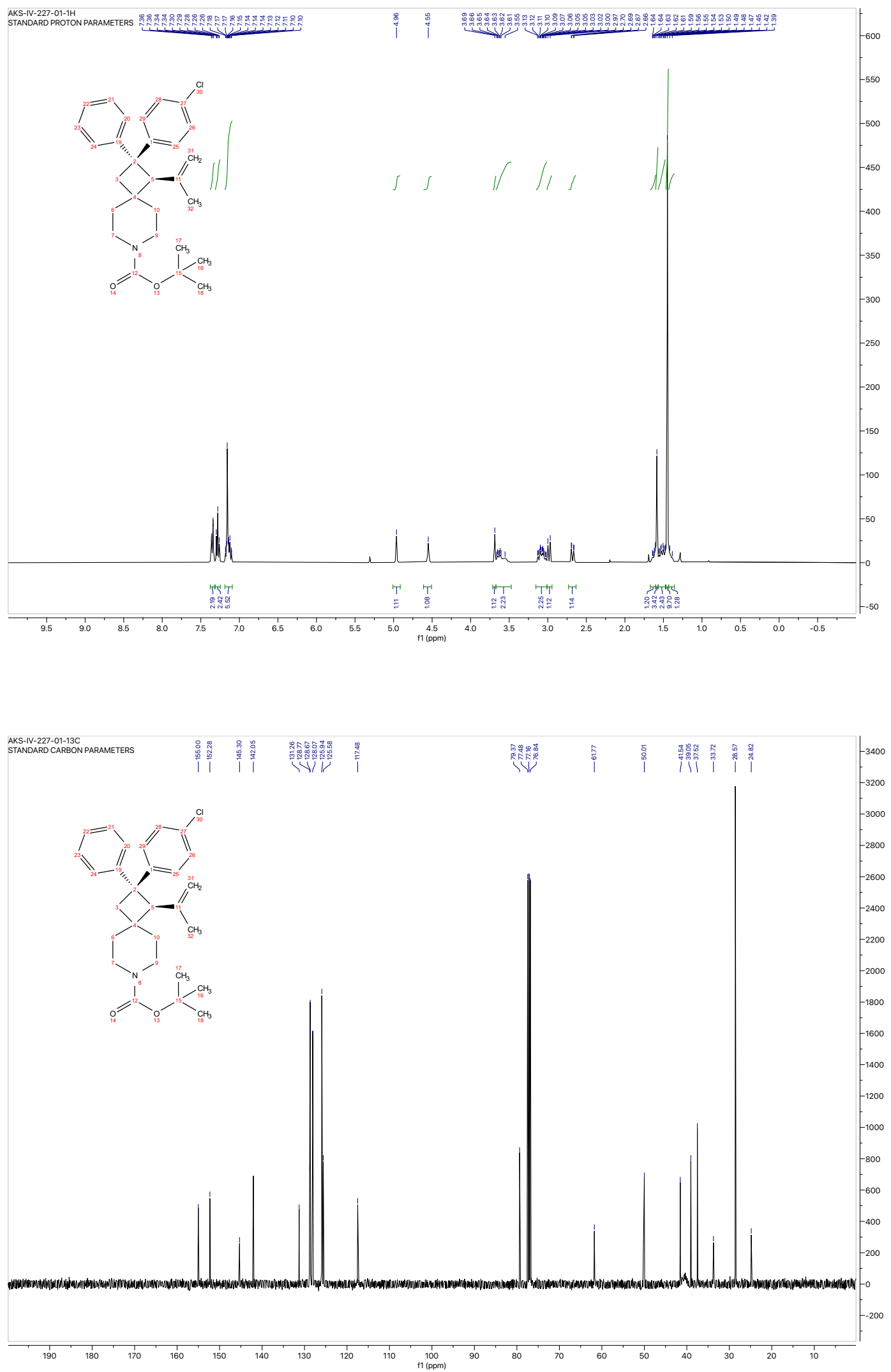

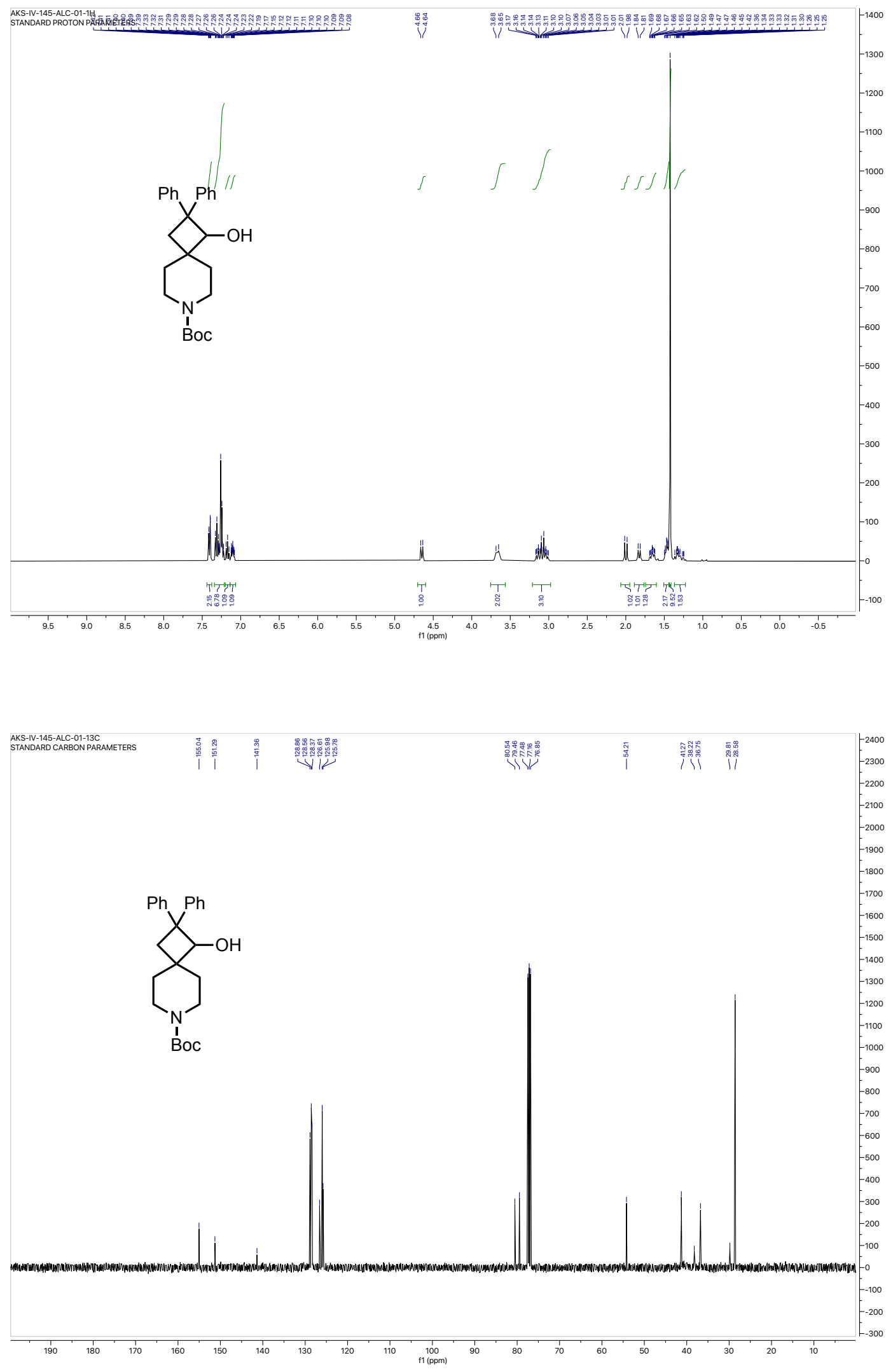

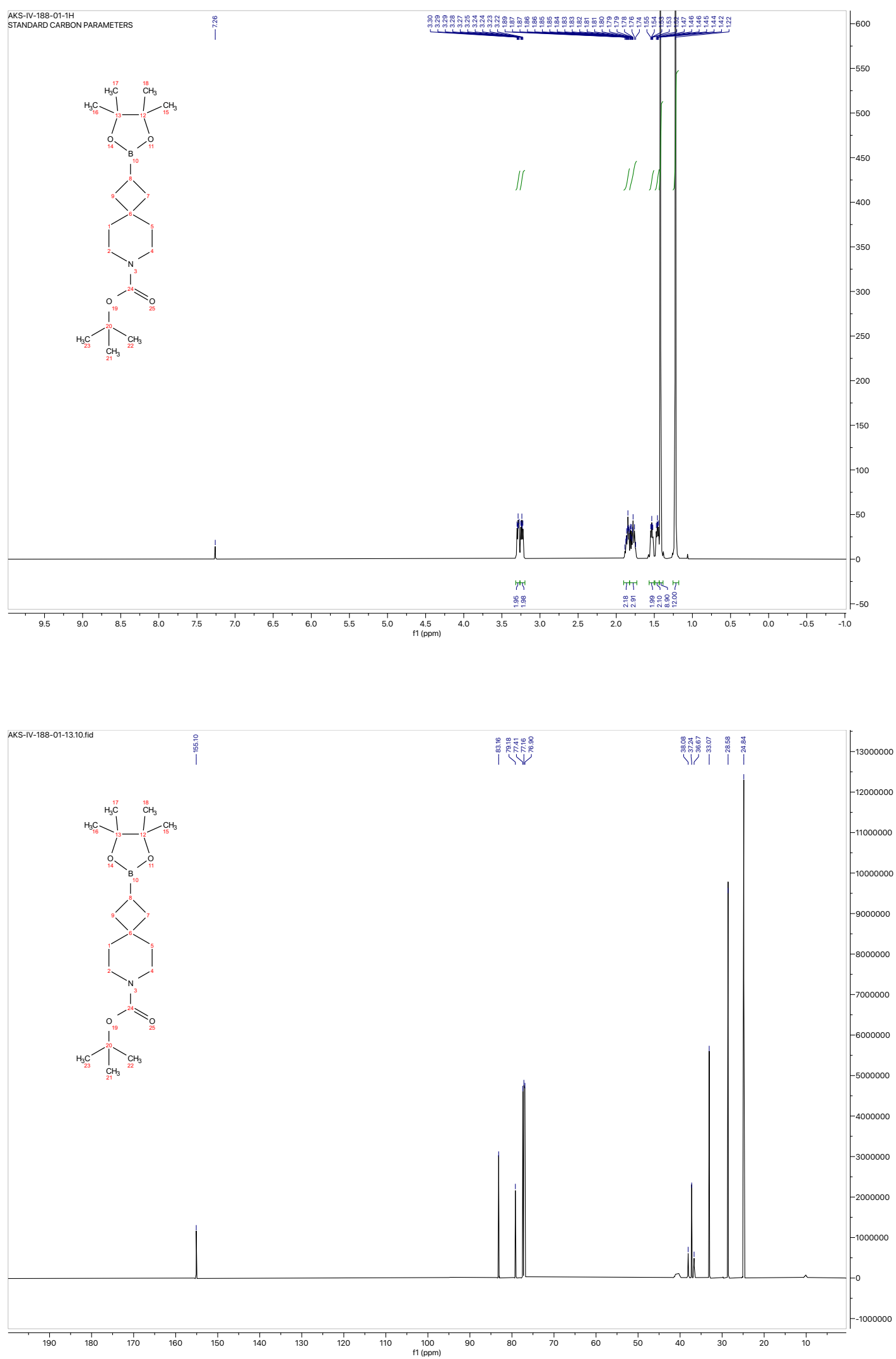


\section{References:}

1. Nóvoa, L.; Trulli, L.; Parra, A.; Tortosa, M., Stereoselective Diboration of Spirocyclobutenes: A Platform for the Synthesis of Spirocycles with Orthogonal Exit Vectors. Angew. Chem. Int. Ed. 2021, 60, 11763-11768.

2. $\quad$ Verdonck, S.; Pu, S.-Y.; Sorrell, F. J.; Elkins, J. M.; Froeyen, M.; Gao, L.-J.; Prugar, L. I.; Dorosky, D. E.; Brannan, J. M.; Barouch-Bentov, R.; Knapp, S.; Dye, J. M.; Herdewijn, P.; Einav, S.; De Jonghe, S., Synthesis and Structure-Activity Relationships of 3,5-Disubstitutedpyrrolo[2,3-b]pyridines as Inhibitors of Adaptor-Associated Kinase 1 with Antiviral Activity. $J$. Med. Chem. 2019, 62, 5810-5831.

3. Bruno, N. C.; Tudge, M. T.; Buchwald, S. L., Design and preparation of new palladium precatalysts for $\mathrm{C}-\mathrm{C}$ and $\mathrm{C}-\mathrm{N}$ cross-coupling reactions. Chem. Sci. 2013, 4, 916-920.

4. Romanski, S.; Rücker, H.; Stamellou, E.; Guttentag, M.; Neudörfl, J.-M.; Alberto, R.; Amslinger, S.; Yard, B.; Schmalz, H.-G., Iron Dienylphosphate Tricarbonyl Complexes as WaterSoluble Enzyme-Triggered CO-Releasing Molecules (ET-CORMs). Organometallics 2012, 31, 5800-5809.

5. Cahiez, G.; Habiak, V.; Gager, O., Efficient Preparation of Terminal Conjugated Dienes by Coupling of Dienol Phosphates with Grignard Reagents under Iron Catalysis. Org. Lett. 2008, 10, 2389-2392.

6. Braconi, E.; Götzinger, A. C.; Cramer, N., Enantioselective Iron-Catalyzed Cross-[4+4]Cycloaddition of 1,3-Dienes Provides Chiral Cyclooctadienes. J. Am. Chem. Soc. 2020, 142, 19819-19824. 